INTERNATIONAL MONETARY FUND

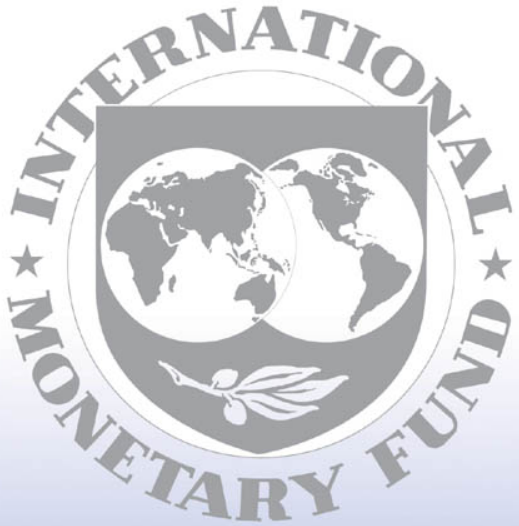

Staff

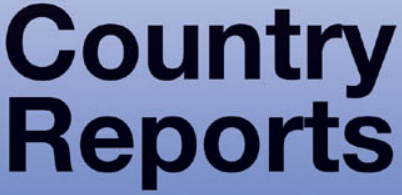




\title{
Kenya: Request for Disbursement Under the Rapid-Access Component of the Exogenous Shocks Facility-Staff Report; Staff Supplement; Press Release on the Executive Board Discussion; and Statement by the Executive Director for Kenya
}

In the context of the request for disbursement under the rapid-access component of the Exogenous Shocks Facility by Kenya, the following documents have been released and are included in this package:

- $\quad$ The staff report for the Request for Disbursement Under the Rapid-Access Component of the Exogenous Shocks Facility, prepared by a staff team of the IMF, following discussions that ended on March 6, 2009, with the officials of Kenya on economic developments and policies. Based on information available at the time of these discussions, the staff report was completed on May 15, 2009. The views expressed in the staff report are those of the staff team and do not necessarily reflect the views of the Executive Board of the IMF.

- A staff supplement on the joint IMF/World Bank debt sustainability analysis.

- $\quad$ A Press Release summarizing the views of the Executive Board as expressed during its May 29, 2009 discussion of the staff report that completed the review.

- A statement by the Executive Director for Kenya.

The document listed below has been separately released.

Letter of Intent sent to the IMF by the authorities of Kenya*

*Also included in Staff Report

The policy of publication of staff reports and other documents allows for the deletion of marketsensitive information.

\author{
Copies of this report are available to the public from \\ International Monetary Fund • Publication Services \\ $70019^{\text {th }}$ Street, N.W. $\bullet$ Washington, D.C. 20431 \\ Telephone: (202) 623-7430 • Telefax: (202) 623-7201 \\ E-mail: publications@imf.org • Internet: http://www.imf.org
}

\section{International Monetary Fund Washington, D.C.}


This page intentionally left blank 
INTERNATIONAL MONETARY FUND

\title{
KENYA \\ Request for Disbursement Under the Rapid-Access Component of the Exogenous Shocks Facility
}

\author{
Prepared by the African Department \\ (In consultation with other departments)
}

Approved by Saul Lizondo and Dominique Desruelle

May 15, 2009

\begin{abstract}
Context: Several exogenous shocks have hit the Kenyan economy since early 2008. These include a drought, rising international food, fuel, and fertilizer prices in the first half of the year, and since later in 2008, weakening capital flows and global demand due to the global financial crisis. These shocks have cut short an economic recovery from the political disturbance of early 2008 and they are weakening the balance of payments, threatening macroeconomic stability, and eroding the gains of the previous five years.
\end{abstract}

Request for support from the Exogenous Shocks Facility (ESF): In light of these shocks, the authorities are requesting disbursement under the Rapid-Access Component of the ESF of SDR 135.7 million (50 percent of quota) to support the balance of payments and adjust to the shocks. The full amount would become available upon Board approval.

Discussions: Discussions were held in Nairobi during February 23-March 6, 2009. The mission met with Prime Minister Raila Odinga, Deputy Prime Minister and Minister for Finance Uhuru Kenyatta, Central Bank Governor Njuguna Ndung'u, other senior officials, representatives of the donor community, and the private sector. The mission comprised Messrs. Atingi-Ego (head) and Yang, Ms. Masha (all AFR); and Mses. Mkusu (SPR) and Zephirin (MCM). Mr. Rogers, Senior Resident Representative participated in the discussions, while Ms. Sayeh joined the mission on the last day.

IMF Relations: The last PRGF arrangement (SDR 150 million) expired in November 2007. The 2008 Article IV consultation was concluded in September 2008, when the Executive Board also discussed the ex post assessment of Kenya's long-term program engagement with the Fund. In concluding the consultation, Executive Directors commended the authorities for maintaining macroeconomic stability in the wake of the post-election turmoil, and for sound macroeconomic policies and progress with economic reforms. They underscored that since downside risks remained, sound policies and continued structural and governance reforms were essential to maintain macroeconomic stability, restore strong growth and advance towards the Millennium Development Goals. Directors broadly concurred with the findings and recommendations of the ex post assessment. They noted that some aspects of the past engagement were disappointing - in particular, the protracted focus on governance issues, and insufficient attention to program ownership. Directors welcomed the improved ownership and performance in recent years. Kenya has accepted the obligations of Article VIII, Section 2, 3, and 4 and maintains an exchange system free of restrictions on payments and transfers for current international transactions. 


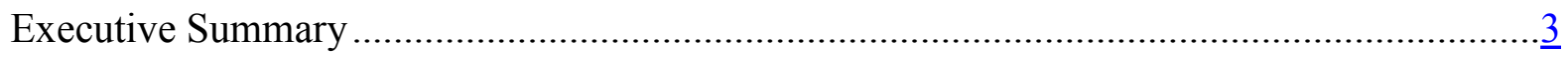

I. Recent Developments and Exogenous Shocks ................................................

II. The Impact of the Exogenous Shocks.......................................................... $\underline{5}$

III. Policy Response, Request for Disbursement under the RAC of the ESF, and Macroeconomic Framework ...................................................................... 10

A. Initial Policy Response and Request for Disbursement under the RAC of the ESF .............................................................................. 10

B. Macroeconomic Framework ............................................................ $\frac{12}{13}$

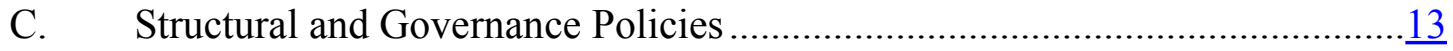

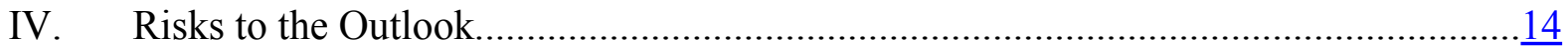

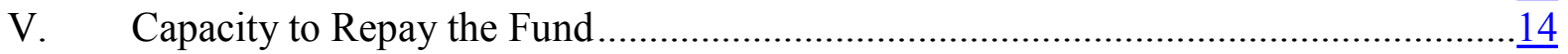

VI. Staff Appraisal ..........................................................................................

Box

1. The Balance of Payments Impact of Exogenous Shocks..........................

Tables

1. Selected Economic Indicators, 2006/07-2013/24 .............................................16

2a. Central Government Financial Operations, 2006/07-2013/14 .............................17

2b. Central Government Financial Operations, 2006/07-2013/14 .................................18

3. Monetary Survey, 2006-10 .......................................................................... $\frac{19}{20}$

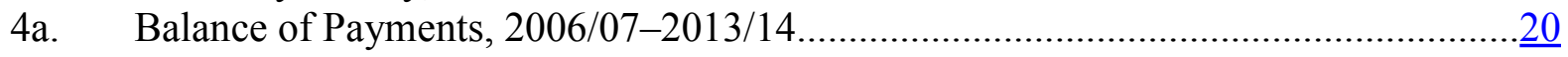

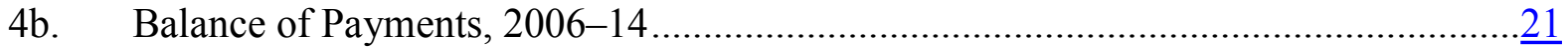

5. External Financing Requirements and Resources..........................................22

6. Financial Soundness Indicators, 2003-08.......................................................23

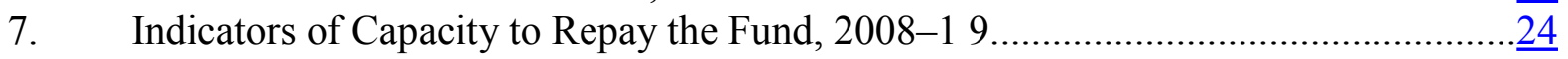

Appendix

Letter of Intent 


\section{EXECUTIVE SUMMARY}

- Kenya's strong economic performance in recent years with real GDP growth of 6 percent on average over 2004-07, has been stalled by a series of exogenous but temporary shocks that hit the economy in 2008. These include a drought; steep increases in international food, fuel and fertilizer prices; the global financial crisis and the associated slowdown in external demand. Together, the shocks have affected economic activity and resulted in the loss of international reserves, eroding the achievements of the previous five years, threatening macroeconomic stability and increasing vulnerability to further shocks.

- $\quad$ The authorities have responded to the shocks swiftly. Monetary policy has been eased to support economic activity; and fiscal policy focused on reprioritizing expenditure. Structural policies have focused on improving the food distribution mechanism for better access to staples for an estimated 10 million food deficient Kenyans; as well as introducing measures to strengthen the banking system to moderate the potential impact of the shocks.

- $\quad$ Recent improvements in Kenya's terms of trade could moderate the overall impact of the shocks on the balance of payments. However, the country will need to substantially increase maize imports, until at least the third quarter of 2009, when the next food harvest is expected. This is expected to further erode official foreign exchange reserves and put pressure on the exchange rate at a time when exports, remittances, and capital inflows are being threatened by the global slowdown.

- $\quad$ The Rapid-Access Component of the ESF would assist Kenya to rebuild foreign reserves in a manner that does not disrupt macroeconomic stability, and bolster market confidence in the difficult period ahead. In addition, Fund support would take place in the context of an increase in donor support.

- $\quad$ The authorities have committed to additional macroeconomic and structural policies designed to further reinforce macroeconomic stability, reduce inflation, and rebuild reserves. Introduction of a sustainable and targeted subsidy framework late in 2009 would improve food security for the most vulnerable households.

- $\quad$ Staff supports the authorities' request for disbursement under the Rapid-Access Component of the ESF. Implementation of the macroeconomic and structural policies would help the authorities achieve their economic objectives. It could also enable Kenya to rally support from donors to address these specific shocks. 


\section{RECENT DEVELOPMENTS AND EXOGENOUS SHOCKS}

1. Sound economic policies and a favorable external environment contributed to Kenya's strong economic performance during 2004-07, when real GDP growth averaged 6 percent, peaking at 7 percent in 2007. Significant progress was made in addressing long-standing weaknesses in public finance management, the financial sector, and the business climate. Some progress was achieved on governance related issues; but much still remains to be done.

2. Against this background, the economy entered 2008 with strong momentum that was stalled by violence related to the December 2007 election. The disturbance soured sentiments towards the tourist industry and took a toll on economic activity in agriculture and manufacturing. In the first quarter of 2008, real GDP contracted by 1.1 percent (year-on-year), on account of strong decline in agriculture and forestry, which shrank by 4.4 percent. Tourist arrivals were down by over 30 percent. Inflation also accelerated due to the supply disruptions and dislocations, and an accommodative monetary policy ${ }^{1}$.

\section{The economy was on its way to} recovery in the second quarter of 2008 but the recovery was stalled by the onset of exogenous shocks. Real GDP growth initially rebounded to 3.4 percent, balance of payments pressures eased, the currency strengthened due to increased capital inflows associated with the Safaricom IPO, which also strengthened the fiscal position. But this was not sustained through the end of the second quarter, as a series of exogenous but temporary shocks hit the economy, putting severe pressure on domestic prices, the external current account, the

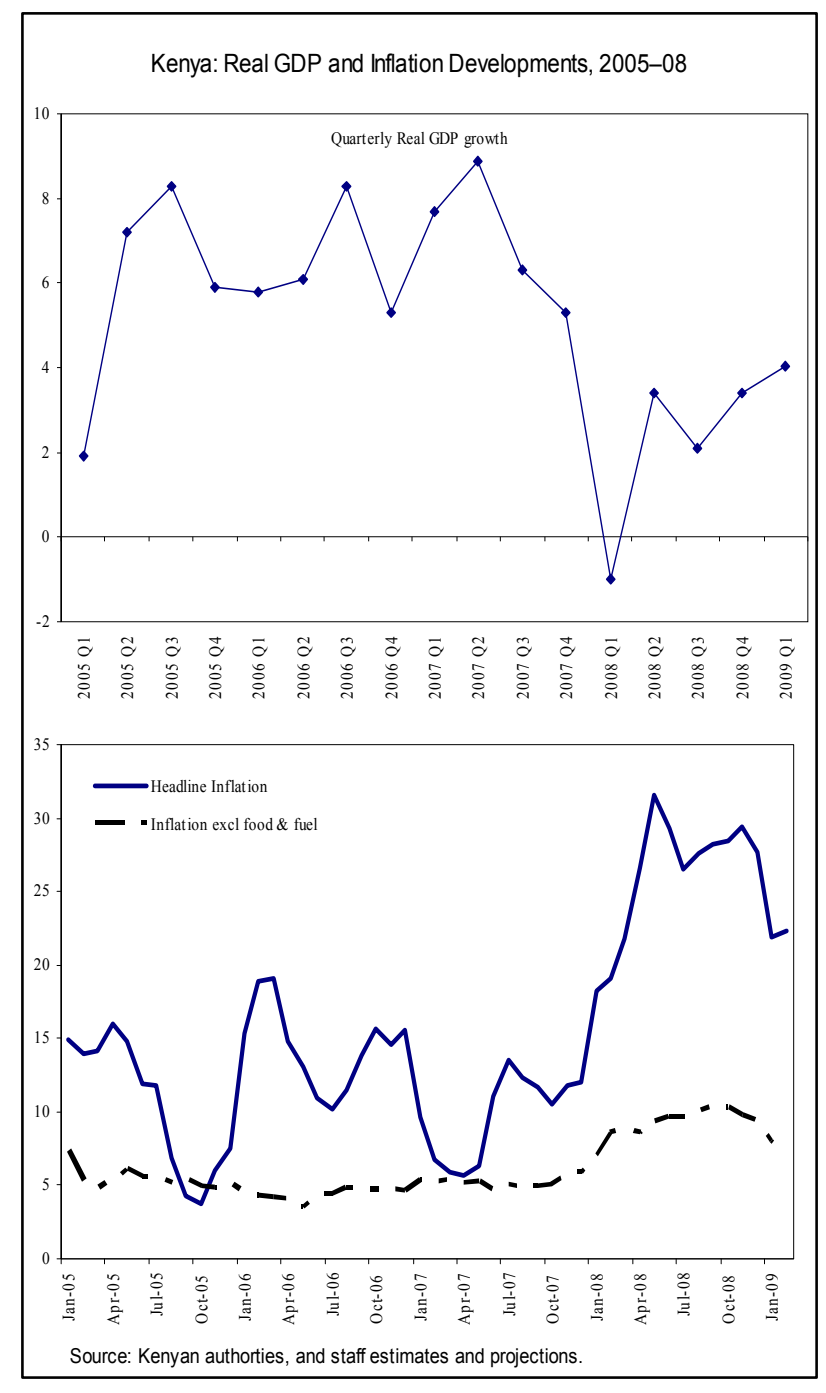

\footnotetext{
${ }^{1}$ The CPI inflation is overstated by a factor of about two, due to methodological problems. STA has provided initial technical assistance to resolve the problems; and to change to a new methodology. See (Country Report No. 08/339).
} 
fiscal position, exchange rate, and international reserves. The shocks include:

- $\quad$ Rising international prices, particularly of fuel and fertilizer. This resulted in low utilization of mechanized land and fertilizer, contributing to lower yields and food supplies, particularly maize, the country's staple crop.

- $\quad$ Poor rainfall in October-November 2008, which exacerbated the decline in maize production.

- $\quad$ Global financial crisis; which intensified in the last quarter of the year curtailed export

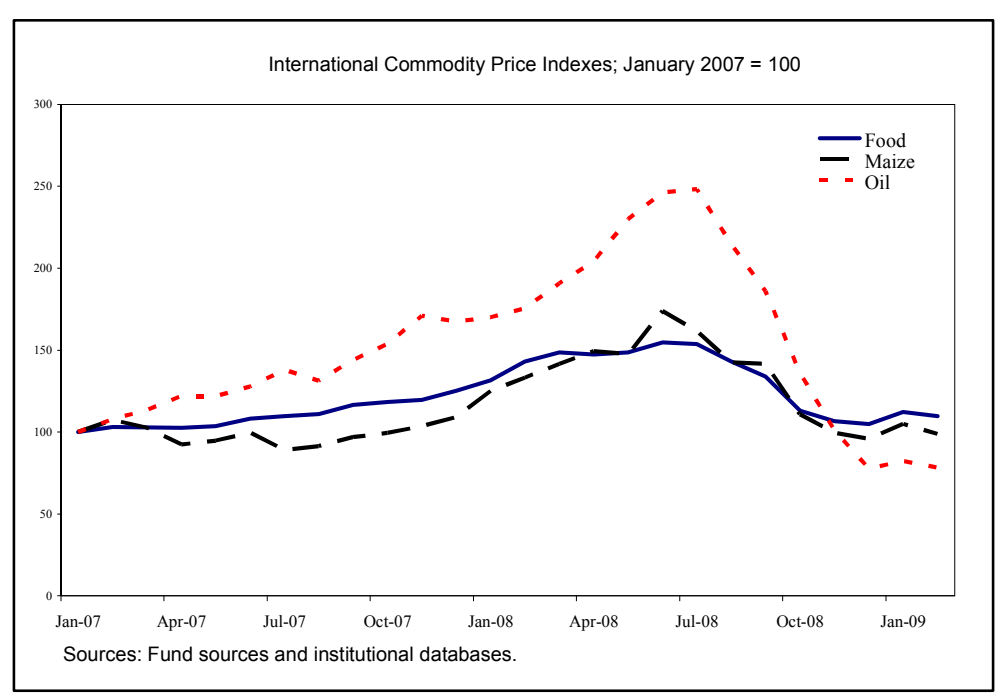
growth, tourism receipts, remittances and private capital flows.

\section{THE IMPACT OF THE EXogenOUS SHOCKS}

4. The exogenous shocks have had a large adverse impact on the balance of payments (Box 1), which has weakened substantially since the first half of 2008/09, and are a primary source of the balance of payments problems. For 2009/10 staff and the authorities project an improvement in the overall balance of payments. While exports earnings, tourism receipts, and remittances would remain depressed, improving terms of trade, largely on account of low oil prices would keep the import bill down, allowing for an improvement in the current account. Moreover, with the expected gradual recovery of the global economy, capital flows are projected to pick up in the first half of 2010, contributing to a modest improvement in the overall balance of payments. But these developments would not be enough to offset the losses incurred in 2008/09. Under these circumstances, the rebuilding of official reserves would be difficult without additional external assistance. 


\section{Box 1. The Balance of Payments Impact of Exogenous Shocks}

Since the first half of $\mathbf{2 0 0 8} / \mathbf{0 9}$, a series of adverse exogenous shocks have affected Kenya and are the primary source of the deterioration of its balance of payments (BOP) and the ensuing financing gap. In 2007/08, Kenya's current account deficit widened owing primarily to rising oil, fertilizers, and other commodity import prices. Nonetheless, thanks to ample net private capital inflows that more than offset the larger current account deficit, the overall BOP was positive, allowing for an accumulation of reserves. The picture has changed since the second half of 2008. Despite the decline in oil prices, substantially lower private capital flows, subdued exports and tourism receipts, and high drought driven maize imports weakened Kenya's estimated 2008/09 and 2009/10 balance of payments, eroding official foreign exchange reserves. As discussed below, staff estimates indicate that exogenous shocks are responsible for at least 70 percent of the US\$ 1.95 billion (61/2 percent of GDP) cumulative deterioration in the BOP during 2008/09-2009/10 compared with 2007/08 (Text Table 1). During this period, gross official financing (mainly project grants and loans) is projected to increase by US\$544 million relative to 2007/08 (Text Table 1). There is a remaining financing gap of about US\$201 million for which the authorities seek financing.

Staff estimates the cumulative adverse impact of exogenous shocks on the BOP during 2008/09 and $2009 / 10$ at $4 \frac{1}{2}-7 \frac{1}{4}$ percent of GDP (US\$1.4-US\$2.2 billion). The estimates are obtained from two alternative methods. These methods use the same assumptions about the non-merchandise items and different assumptions about merchandise items. For the non-merchandise items - tourism, remittances receipts and private capital flows, the projected decline relative to $2007 / 08$ is assumed to be driven by the global financial crisis. For the merchandise items, the two methods use different assumptions about the volume of imports and exports. As the non-merchandise items are identical in the two alternatives, the difference between the two estimates of the adverse impact of the exogenous shock reflects differences in trade volume assumptions. The upper estimate, $7 \frac{1}{4}$ percent of GDP, takes into account the full effect of price and volume changes, using projected prices and volumes for 2008/09 and 2009/10 (alternative A). The lower estimate, $4 \frac{1}{2}$ percent of GDP, reflects an impact on the trade balance that takes into account price changes only, assuming volumes remain constant at their 2007/08 levels (alternative B). The decline in net FDI and short-term capital flows, the single most important driver of the BOP deterioration, represents over 7 percent of GDP.

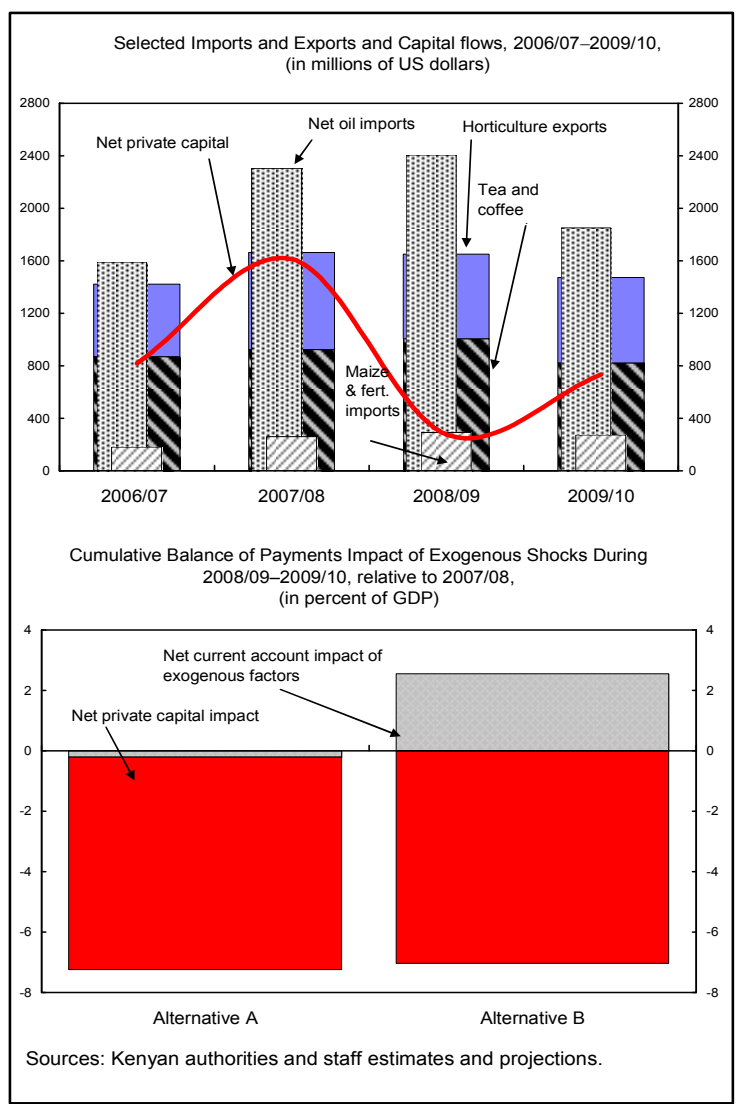

The methodology based on a constant volume assumption tends to underestimate the magnitude of the exogenous shocks' impacts. Specifically, it does not allow for quantity adjustments in response to changing prices, external demand or changing domestic factors, including the drought-driven need for increased maize imports for which the government appealed to donors for support, and the need for higher oil import volumes as the economy grows.

Nonetheless, in both alternatives, the cumulative impact of the exogenous shocks during 2008/092009/10 is substantial. As indicated above, it is responsible for the estimated financing gap during the same period, as well as the decline in gross official reserves to less than 3 months of imports by end 2009/10, from over $3 \frac{1}{2}$ months of imports at end-2007/08. 
Text Table. Estimated Impacts of Exogenous Shocks on the Balance of Payments, 2008/09-2009/10 (In millions of US dollars, unless otherwise indicated)1

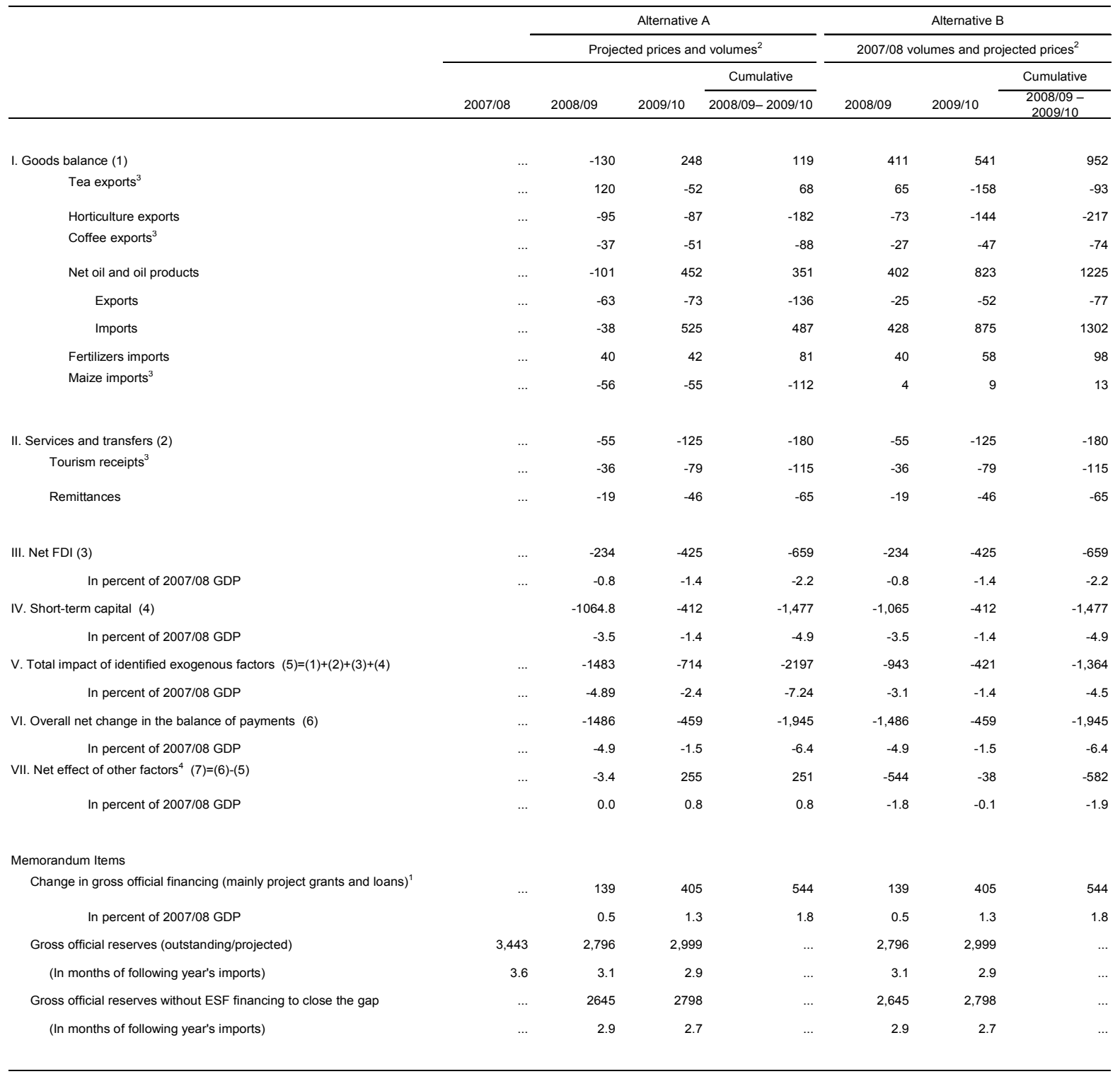

${ }^{1}$ The fiscal year runs from July to June. Figures shown reflect changes relative to the reference year 2007/08.

${ }^{2}$ Actual prices or volumes refer to $2007 / 08$ and projected ones refer to outer years.

${ }^{3}$ The impact of the post-election violence is excluded from exogenous shocks. It is estimated that without the post-election disruption to production, tea and coffee exports in $2007 / 08$ would have been 5 percent higher than observed, and maize imports in 2008/09 would have been 10 percent lower than currently projected. Also, the decline in tourism flows registered in the first half of 2008 is attributed to post-election violence.

${ }^{4}$ The net effect of other factors is a residual, reflecting changes in the balance of payments not singled out in the Table. In alternative A where the impact of both price and volume changes is captured, this effect is positive, reflecting in part larger official flows relative to 2007/08. However in alternative B, it is negative because it includes a weaker trade balance effect, not captured as such owing to the constant volume assumption.

\section{(CInternational Monetary Fund. Not for Redistribution}


5. The impact of the shocks on monetary and exchange rate developments has been pronounced. At end 2008, broad money and aggregate credit growths slowed, consistent with the lower than projected real income. During the first half of the 2008, the exchange rate appreciated as capital inflows increased. However, by the end of the year, the combined effect of the global financial crisis and the strengthening of the US dollar resulted in increased capital outflows, with the exchange rate depreciating with respect to the US dollar by 24 percent in the year. The strengthening of the US dollar against other currencies in Central Bank of Kenya (CBK) foreign assets portfolio also contributed to some revaluation loss. The shilling has depreciated in nominal effective terms in the second half of 2008, and has shown some downward pressure since then. In real effective terms, it depreciated by about 10 percent since the beginning of 2008 .

6. The global financial crisis has so far had minimal effects on the banking system, but the impact on the capital market has been pronounced. The banks' regulatory capital asset ratio (CAR) was above 18 percent as at-end December 2008 and all banks met the minimum capital and liquidity requirements. Initial stress tests indicate that in the event of a deterioration in loan performance three banks whose CAR's are just above the required level would need to replenish their capital. These three banks account for 13 percent of the total banking system deposit liability. Going forward, however, the quality of loan portfolios is likely to deteriorate in the months ahead, particularly in banks exposed to the tourism and export sectors. In the capital market, as portfolio investor confidence waned in the second half of the year, equity prices on the Nairobi Stock Exchange fell by some 40 percent, in line with developments in
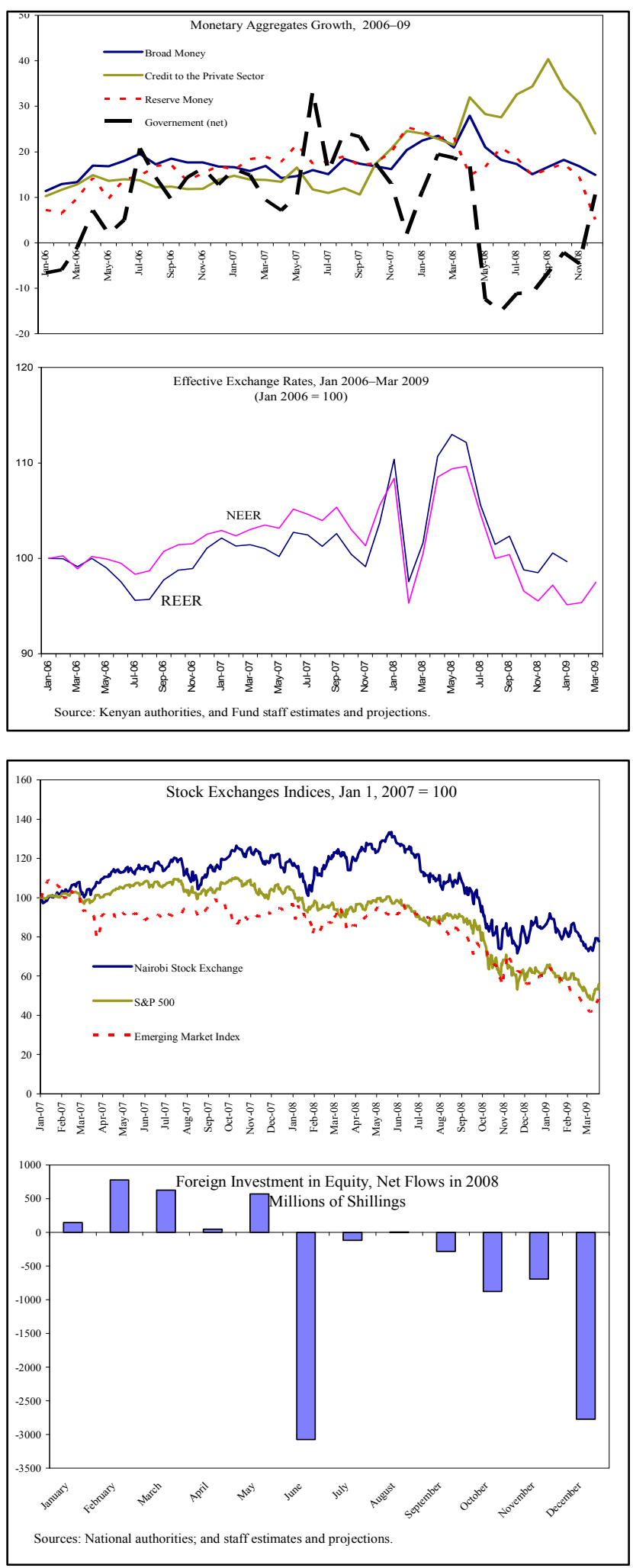
major stock exchanges. The exit of foreign investors from the stock exchange was particularly pronounced in the last quarter of 2008.

7. The exogenous shocks have weakened the government's fiscal outlook. After a strong performance in 2007/08 despite the political events of early 2008, revenue collections were below the budgeted level during the first 9 months of fiscal 2008/09 (July/June), reflecting a slowing economy. However, expenditures were also lower than budgeted on account of slow implementation of externally financed projects. Unfortunately, the unfavorable economic environment led to the postponement of planned sovereign bond issue and privatization. Together with the need to finance necessary drought mitigation measures, the postponement has created the need for domestic financing of 4 percent of GDP.

\section{The drought and higher food prices have had a pronounced impact on the poor and vulnerable.}

According to the government and the $\mathrm{UN}$, due to the drought, food insecurity

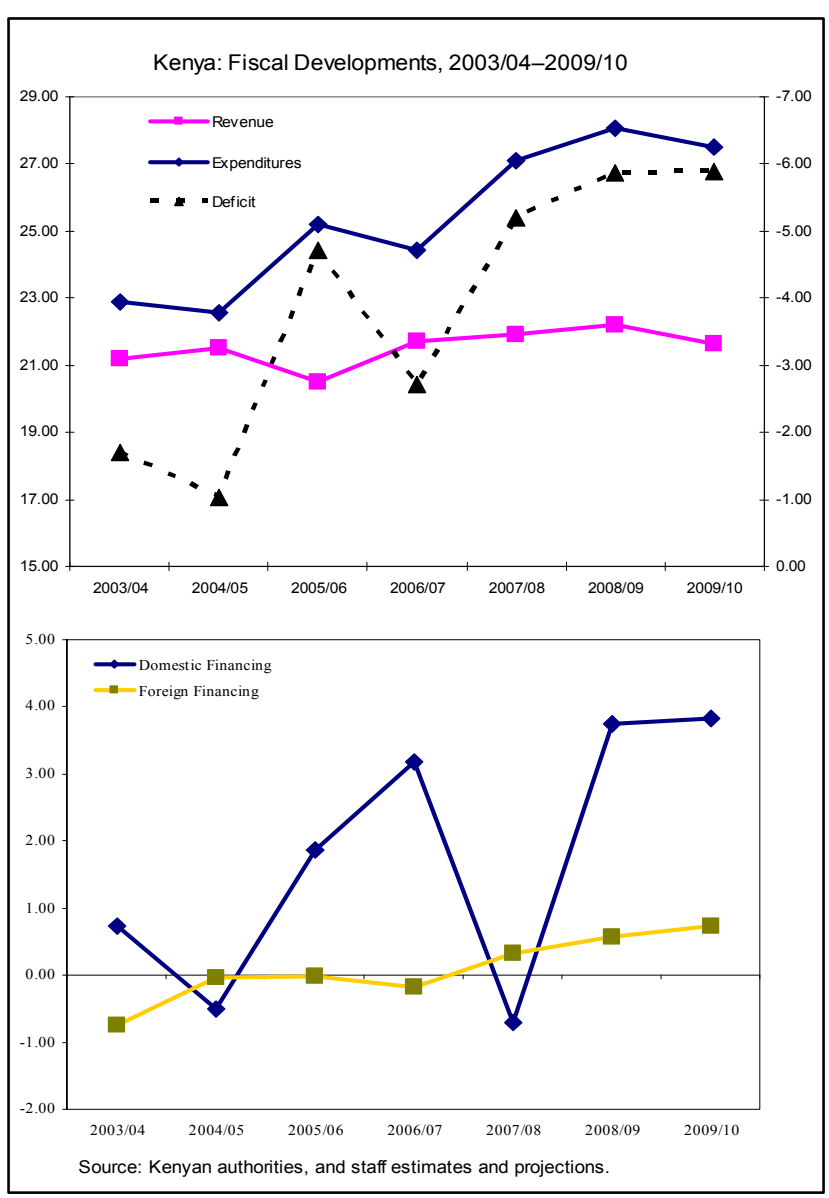
has increased by about 47 percent, and now stands at around an estimated 10 million people. Food insecurity has deepened in the agricultural areas due to the drought, while in the urban areas the reduction in disposable income was an additional factor, resulting in a decline in household food consumption in outskirts of Nairobi and Mombasa.

9. Jointly, the shocks, though temporary, have constrained economic growth and fuelled inflationary pressures. Real GDP growth is now estimated at about 2 and 3 percent for 2008 and 2009, respectively. Headline inflation ${ }^{2}$, which accelerated in 2008 Q1 due to rising global food and fuel prices, was sustained by pronounced currency depreciation, despite a reduction in the growth of monetary aggregates. By end-2008, inflation reached 27.7 percent, averaging 26.2 percent for the year as a whole. Underlying inflation increased from 5.3 percent at the end of 2007 to 9 percent in December, but has since declined.

\footnotetext{
2 The interpretation of inflation statistics is complicated by methodological problems that lead to substantial overestimation, as explained above (see footnote 1).
} 


\section{Policy Response, ReQuest for Disbursement Under the RAC OF THE ESF, AND MACROECONOMIC FRAMEWORK}

\section{A. Initial Policy Response and Request for Disbursement under the RAC of the ESF}

10. Several measures were introduced to mitigate the initial impact of the drought on the vulnerable, as well as to moderate the macroeconomic impact of other exogenous shocks.

- $\quad$ Mitigation of the impact of high food prices. The government introduced a maize subsidy scheme in late 2008. The National Cereals and Produce Board sold maize to millers at below market prices, with the savings passed on to consumers. However, this scheme depended on farmers selling to the National Cereal and Produce Board (NCPB) at below-market prices, which they refused to do. Consequently the scheme was abandoned in early 2009. At the same time, to lower the market price of imported maize, the government has suspended VAT and customs duties. The government has begun working with the World Bank on a well-targeted subsidy program to be introduced by the end of 2009. Additional fiscal policy measures are planned for implementation in the revised 2008/09 budget.

- Declaration of food emergency. The government declared a food emergency for an estimated 10 million food-insecure Kenyans, and appealed to donors for US\$410 million in food and agriculture-related support, As part of the response of the donor community, the World Food Program has appealed for over US\$ 200 million, and other donors have indicated their willingness to support this appeal. Should realized donor

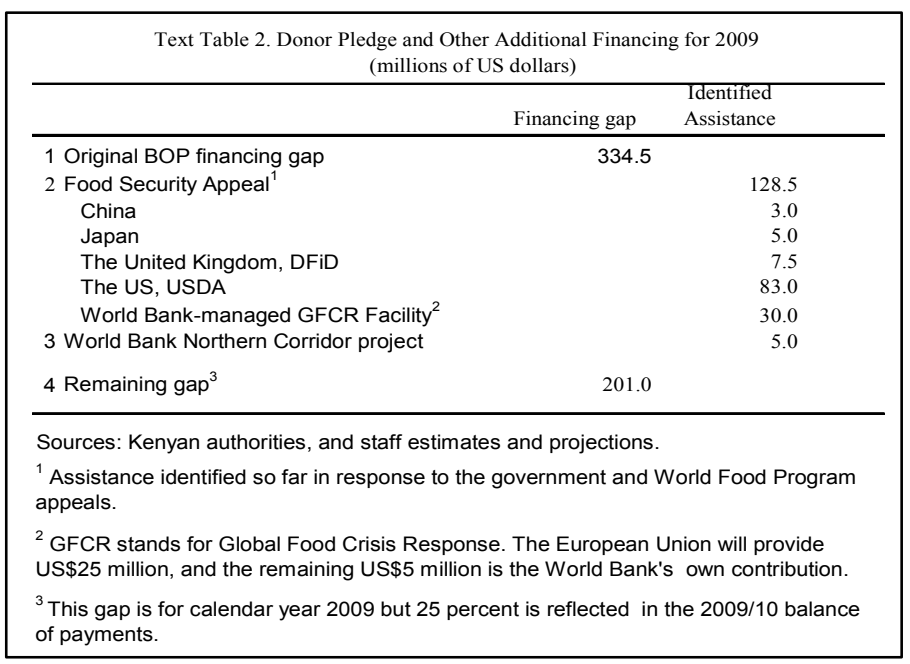
support exceed US\$133.5 million, the government plans to increase agricultural inputs related imports.

- $\quad$ Running down of reserves. In the wake of the financial crisis in the third quarter of 2008, CBK did not go to the market to replace foreign exchange sold to government to finance external payments, to avoid putting further pressure on the exchange rate. Official reserves therefore continued to decline. 
- $\quad$ Easing of the stance of monetary policy. An accommodative monetary policy stance that began in early 2008 through increased reverse repurchase operations by the CBK, intensified in mid year, and continued into the first quarter of 2009. As of March 2009, CBK had cut the policy rate by a total of 75 basis points, and reduced the cash reserve requirement by 1 percentage point.

Despite these efforts to inject liquidity, reserve money, the CBK's operational aggregate remains well below target. Nonetheless, short-term interest rates have fallen since December, with 91-day Tbill, repo and interbank rates standing at about 7.4 percent,

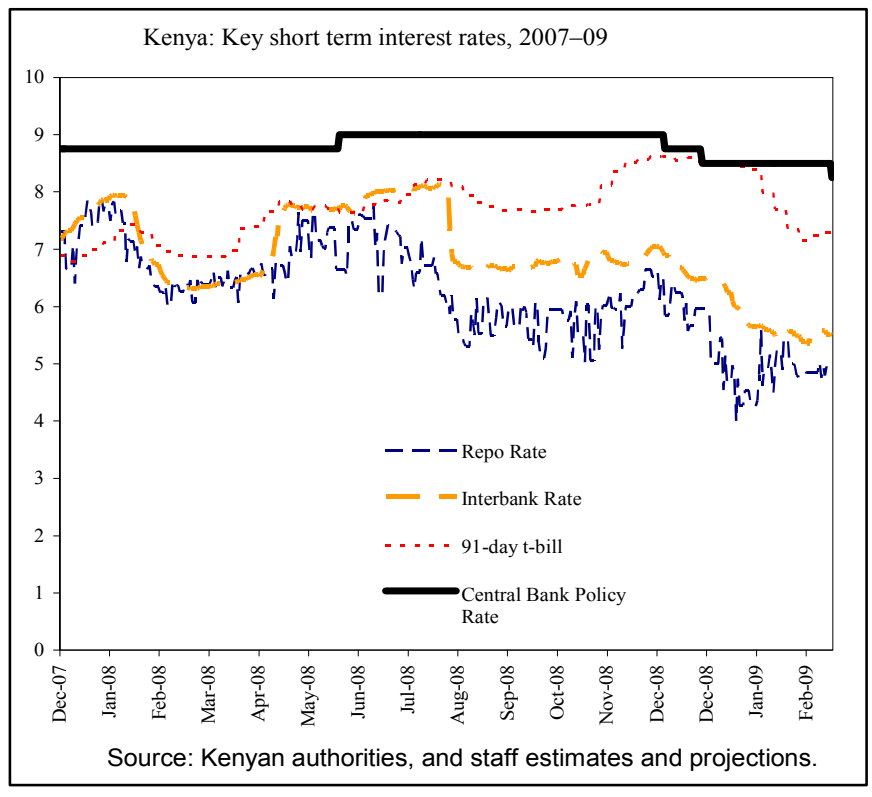
4.3 percent and 5.5 percent respectively, at mid-March.

- $\quad$ Enhancing financial sector surveillance CBK stepped up supervision of all banks, and a special inspection of a major international bank in order to better monitor possible risks to the financial sector arising from the global financial crisis and the economic slowdown. Additional reporting standards, in particular of nonperforming loans by sector was introduced.

11. Despite these policies, the impact of the shocks poses several challenges to the economy. Food price increases are not expected to dissipate at least until after the third quarter, when the long rains are expected to boost food supplies. Thus, there would be need to increase imports of maize through August 2009 to ensure food availability and stabilize domestic prices. This is expected to further erode official foreign exchange reserves and put pressure on the exchange rate, at the time when the prospects for exports, remittances, and capital inflows are being threatened by the global slowdown.

\section{Against this background, the authorities have requested Fund support under the Rapid-Access Component of the ESF in the amount of SDR135.7 million, or} about US\$201 million. The requested access would close the cumulative financing gap estimated for 2008/09 and 2009/10, while enabling Kenya to start rebuilding foreign reserves which are estimated to fall by 23 percent or almost US $\$ 800$ million between 2007/08 and 2008/09 in the absence of financing under the ESF. The request for 50 percent of quota is justified because it would enable Kenya to step up its reserve build up in a way that does not disrupt the domestic financial market or cause macroeconomic instability. The facility would therefore also enable Kenya to bolster further market confidence in the difficult period ahead. 


\section{B. Macroeconomic Framework}

13. The authorities recognize that supportive macroeconomic policies and structural reforms are needed to reduce future balance of payments vulnerabilities and set the economy on the path to strong economic growth. Therefore, they plan to implement a set of macroeconomic and structural policies aimed at maintaining macroeconomic stability, reigning in inflation and sustaining economic growth and poverty reduction.

\section{Monetary, Exchange Rate, and Financial Sectors Policies}

14. Monetary and exchange rate policy would focus on reducing inflation, providing sufficient liquidity to support economic activity while allowing for a gradual build up in official reserves. In the recent past, despite CBK's countercyclical policy, including lowering of interest rates, the growth of monetary aggregates continue to slow down, signaling uncertainties about money demand. Going forward therefore, the CBK's monetary program for the rest of 2008/09 and 2009/10 targets money growth at a rate only moderately higher than nominal GDP growth in the short run, while focusing on the objective of achieving a 5 percent inflation rate. The projected broad money growth of 13 percent in 2008/09 and 15.8 percent in 2009/10 would allow for adequate expansion of domestic credit to support economic activity without putting pressure on prices.

15. Consistent with the programmed money supply growth for $2009 / 10$ is a foreign reserve accumulation path that enables CBK to gradually rebuild official reserves without jeopardizing the inflation objective. Under the program, CBK would resume foreign exchange purchases in the market and official reserves would increase gradually, from around US\$2.6 billion in March 2009 to almost US\$3.0 billion by endJune 2010, though in months of imports, there is a slight decline during the same period.

16. The authorities reaffirmed their commitment to a market-determined exchange rate. $\mathrm{CBK}$ will continue to maintain a managed float exchange rate regime to allow the exchange rate to reflect the economic fundamentals with interventions limited to smoothing out excessive volatilities in the exchange rate, and rebuilding reserves as indicated above.

17. To address the increased risks to the financial sector, the CBK is intensifying its oversight to ensure that banks are correctly classifying loans and making adequate and timely provision for bad and doubtful loans. CBK will continue to monitor carefully the liquidity conditions while implementing appropriate measures to deal with any perceived stress in the banking sector. In this regard, CBK has asked for technical assistance in developing a contingency plan for the financial sector. In the meantime, the banks are tightening their credit standards to mitigate the risks. 


\section{Fiscal Policy}

18. The fiscal framework aims to strike the right balance between supporting growth and maintaining medium-term debt sustainability. Recent progress made in reducing public debt to GDP ratios gives the government some room to increase domestic borrowing in the short run to accommodate revenue and financing shortfalls. Thus, in line with the recommendations of the 2008 Article IV consultation, there would be a temporary departure from the medium-term debt-to-GDP ratio path, but smaller fiscal deficits over the medium term as the economy recovers would ensure debt sustainability. An updated debt sustainability analysis shows that Kenya continues to face low risk of debt distress despite the increased borrowing in the short run. Moreover, under the proposed monetary program, and considering the flight to safety by commercial banks, there is limited risk that increased government borrowing would crowd out credit to the private sector, which is likely to remain weak in the short run.

19. In response to the financing shortfall noted earlier, the authorities plan to reprioritize expenditures and increase domestic borrowing. A freeze on recruitments has been announced; some projects have been deferred to the next financial year, and some recurrent expenditures, such as those on travel and goods and services, have been cut. However, given the magnitude of the shortfalls and even with drought intervention mostly financed by donors, domestic borrowing on the order of 4 percent of GDP is considered necessary in 2008/09 to protect key expenditures on infrastructure and poverty reduction, as well as to support aggregate demand against the backdrop of a slowing economy. Nevertheless, the overall budget deficit is projected at 5.2 percent of GDP, slightly lower than the originally budgeted 5.3 percent of GDP, but higher than the 3.6 percent of GDP in $2007 / 08$. For 2009/10, facing a similarly unfavorable economic outlook, the authorities intend to continue allowing automatic stabilizers to operate, with an expected fiscal deficit of 5.4 percent of GDP and total net domestic borrowing of about 4.2 percent of GDP in the absence of any sovereign bond issuance. The authorities continue to hope that they will be able to issue a sovereign bond of US\$500 million (1.5 percent of GDP) in 2009/10 to finance key infrastructure projects. Should this issuance take place, which may prove difficult, they would reduce domestic borrowing by an equivalent amount.

\section{Structural and Governance Policies}

20. The macroeconomic framework would be supported by appropriate structural and governance reform policies, to improve long-term growth potential. The major areas of focus, as indicated in the authorities' Letter of Intent are fiscal, monetary, financial and governance reforms. In the fiscal sector, they plan to focus on improved public financial management and related areas. On monetary and financial sector issues, they plan to complete revisions to the CPI that takes into account international best practice in terms of methodology, as well as new weights and expanded coverage of items in the basket. They also plan to undertake the Financial Sector Assessment Program (FSAP) update in the Fall of 2009. There are also plans to continue strengthening the governance agenda in the context of the updated Governance Action Plan (GAP). 
21. A comprehensive reform of the subsidy program to more effectively target the poor is also planned. The new program to be introduced by December would better target the poor, reduce the impact of food prices on the vulnerable, and be transparent in its operations and implications for fiscal costs. In addition, the current ban on maize exports would be lifted as soon as the targeted subsidy program begins to work. The food situation would be reassessed in August with a view of extending the current suspension of import duties on maize imports, if needed, and there are also plans to maintaining a full passthrough of world petroleum prices.

\section{Risks To The OUTLOOK}

22. Staff projects that economic growth is likely to remain low in 2009/10. While barring abnormal weather, agriculture is expected to rebound from the poor harvest in 2008, export, remittances and capital flows are likely to remain depressed in line with global developments; constraining domestic demand and growth. Therefore, risks to the outlook are tilted to the downside and staff currently project that GDP would grow by about 3 percent in 2009/10. Worse than anticipated global economic developments would create substantial headwinds, further depressing merchandise exports, tourism, remittances, and capital flow. These would further restrain growth and weaken the balance of payments.

23. There are also risks from the domestic political situation, as tensions within the coalition government could threaten political stability. While the coalition has provided some stability for economic recovery, there have been tensions about the terms of the power-sharing arrangement in recent times. In addition, the coalition is yet to start to address key constitutional and electoral issues which fuelled the violence, as well as the thorny issues of land grievances. A breakup of the coalition could create further political tensions, which would impact negatively on economic growth.

\section{CAPACITY TO REPAY THE FUnd}

24. Kenya has adequate capacity to repay the Fund. The country has a relatively low stock of external debt, and the debt sustainability analysis places Kenya at a moderate risk of debt distress. Assuming strong implementation of the supporting macroeconomic polices for the program, the NPV of public and publicly guaranteed external debt is projected at 47.9 percent of exports of goods and services; and 11.8 percent of GDP by end 2009. Projected repayment to the Fund will remain under 0.5 percent of exports of goods and services over the medium-term. In preparation for the purchase of Fund resources, CBK has initiated an update of the safeguard assessment.

\section{Staff Appraisal}

25. Kenya has been hit by a series of exogenous but temporary shocks. These include high international food, fuel, and fertilizer prices, and poor rainfall subsequently resulting in poor harvests; the global financial crisis and the associated slowdown in global external demand. The shocks have resulted in a temporary loss of reserves, eroded the 
achievements of the past five years, weakened macroeconomic stability and increased vulnerability to further shocks.

26. The authorities' initial response to the shocks has been strong, and have allowed for temporary moderation in the impact. VAT and customs duty were suspended to lower the cost of maize imports, and increase food availability. Monetary policy was eased to support economic activity, and fiscal policy has been largely accommodating without endangering debt sustainability. Together, these policies have allowed Kenya to achieve a moderate level of economic activity during the global economic slowdown.

27. The authorities have also committed to additional macroeconomic and structural policies designed to reinforce macroeconomic stability, reduce inflation, and rebuild reserves. A gradual reserve accumulation path consistent with the desired reserve money growth would ward off undue volatility in the exchange rate, and disruption in the foreign exchange market. Reserve accumulation would allow for weathering of future shocks to the economy. Fiscal policy would provide a stimulus to the economy and protect key infrastructure and social spending while ensuring sustainable debt to GDP ratio over the medium term.

28. Staff welcomes the authorities' commitment to advancing broad-based structural and governance reforms to ensure strong economic growth over the medium and long term. Priority will be given to the reforms of public finance management, governance, and financial sector which are much needed to compliment sound macroeconomic policies. Introduction of a targeted subsidy framework would address the food deficit in an efficient manner.

29. Staff supports the authorities' request for disbursement under the RapidAccess Component of the ESF, noting that the medium-term outlook is not favorable to the rebuilding of reserves to the pre-crisis level. The requested access would help the authorities achieve their economic objectives and close the projected financing gap while enabling the country to get support from other donors. The authorities are strongly encouraged to implement their policy package to reduce the economy's vulnerability to adverse shocks, monitor developments closely, and be ready to take actions that may be needed to strengthen economic performance and bolster market confidence should risks materialize.

30. There are risks to the macroeconomic program. However, in light of the authorities' track record in macroeconomic management, and their commitment to sound policies, the program has a good chance of success. 
Table 1. Kenya: Selected Economic Indicators, 2006/07-2013/2014

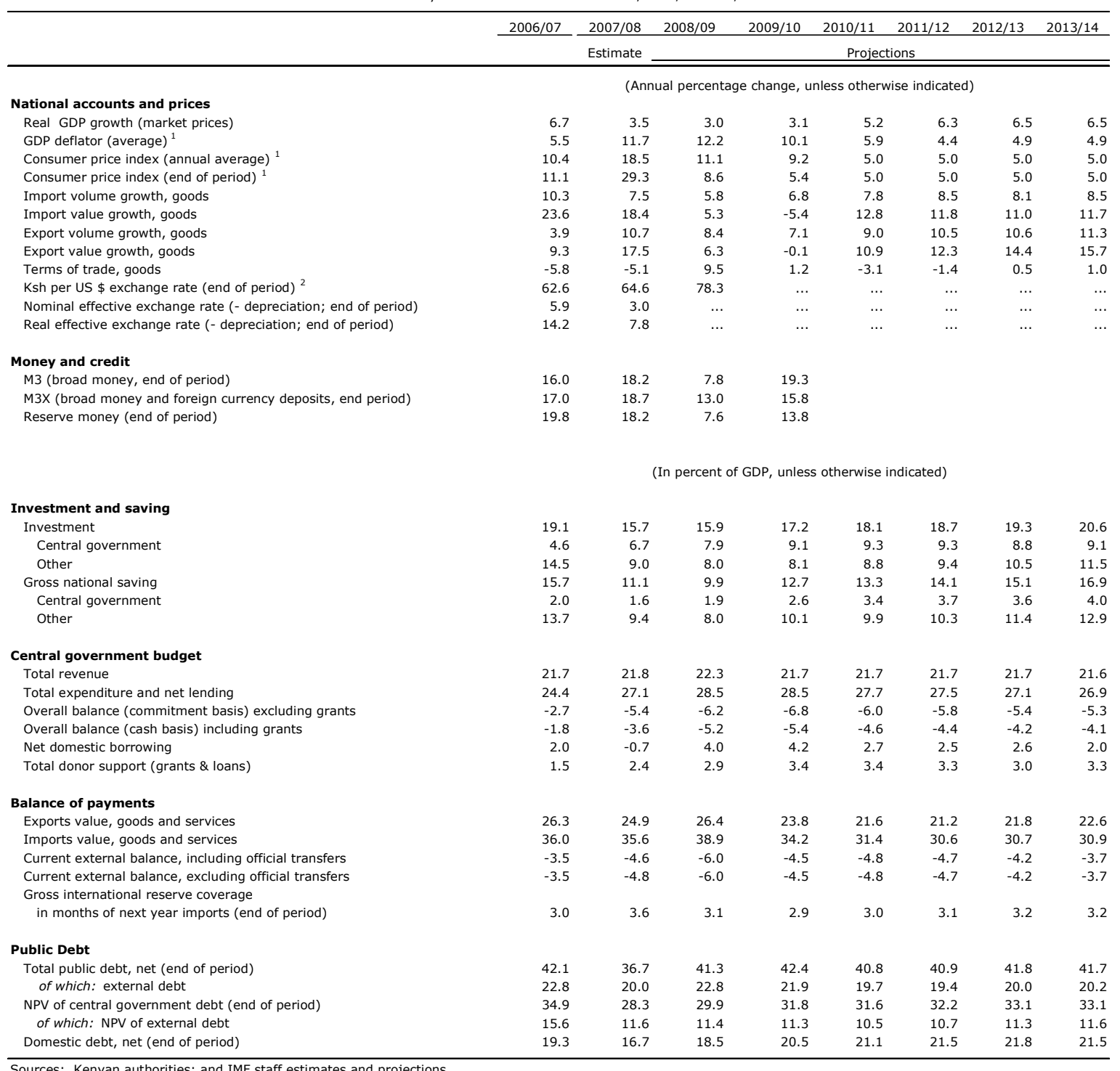

${ }^{1}$ Up to 2007/08, GDP Deflator and Consumer Price Index are overestimated.

${ }^{2}$ Actual as of May 15, 2009. 
Table 2a. Kenya: Central Government Financial Operations, 2006/07-2013/14 ${ }^{1 /}$

\begin{tabular}{|c|c|c|c|c|c|c|c|c|c|}
\hline & $2006 / 07$ & $2007 / 08$ & 2008 & $3 / 09$ & $2009 / 10$ & $2010 / 11$ & $2011 / 12$ & $2012 / 13$ & $2013 / 14$ \\
\hline & Actual & Est. & Budget & $\begin{array}{c}\text { Staff } \\
\text { Projection }\end{array}$ & $\begin{array}{c}\text { Staff } \\
\text { Projection }\end{array}$ & & Projec & ctions & \\
\hline & & (In b & lions of Ken & yyan shillings & unless otherw & se indicated & & & \\
\hline Revenue & 373.0 & 432.2 & 512.7 & 510.7 & 565.1 & 629.5 & 699.6 & 780.0 & 869.5 \\
\hline Income tax & 131.5 & 165.5 & 194.0 & 193.3 & 217.8 & 242.6 & 269.9 & 301.6 & 337.2 \\
\hline Import duty (net) & 27.5 & 32.9 & 36.5 & 36.5 & 41.6 & 44.8 & 48.8 & 51.8 & 56.6 \\
\hline Excise duty & 56.4 & 61.9 & 72.9 & 68.9 & 75.2 & 84.2 & 94.7 & 104.8 & 116.0 \\
\hline Value-added tax & 96.3 & 111.9 & 133.9 & 128.6 & 141.3 & 158.7 & 176.2 & 198.7 & 222.2 \\
\hline Investment income & 6.6 & 3.1 & 5.7 & 7.9 & 6.7 & 7.5 & 8.3 & 9.2 & 10.3 \\
\hline Other & 28.4 & 29.8 & 34.7 & 38.1 & 41.0 & 45.7 & 50.7 & 56.6 & 63.3 \\
\hline Ministerial and Departmental Fees (AIA) & 26.4 & 27.1 & 35.1 & 37.4 & 41.4 & 46.1 & 51.2 & 57.2 & 63.9 \\
\hline Expenditure and net lending & 419.5 & 538.9 & 673.0 & 653.8 & 741.7 & 804.8 & 886.0 & 972.8 & $1,082.2$ \\
\hline Recurrent expenditure & 339.2 & 403.4 & 471.7 & 467.5 & 497.7 & 529.6 & 579.3 & 649.5 & 709.6 \\
\hline Interest payments & 42.5 & 47.9 & 56.7 & 53.9 & 66.1 & 74.8 & 83.8 & 96.1 & 102.3 \\
\hline Domestic interest & 36.9 & 42.2 & 49.4 & 47.8 & 58.0 & 66.7 & 74.1 & 81.7 & 88.3 \\
\hline Foreign interest due & 5.7 & 5.7 & 7.2 & 6.1 & 8.2 & 8.1 & 9.7 & 14.3 & 14.0 \\
\hline Wages and benefits (civil service) & 127.3 & 146.0 & 162.0 & 158.8 & 173.6 & 189.5 & 203.8 & 227.7 & 254.4 \\
\hline Civil service reform & 1.4 & 0.8 & 0.2 & 0.1 & 0.2 & 0.2 & 0.2 & 0.2 & 0.2 \\
\hline Pensions, etc. & 20.4 & 24.1 & 27.1 & 26.1 & 30.0 & 34.4 & 38.8 & 42.7 & 46.5 \\
\hline Other & 119.0 & 139.7 & 171.4 & 179.8 & 178.2 & 178.9 & 200.8 & 225.5 & 242.5 \\
\hline Defense and NSIS 2/ & 28.7 & 44.9 & 50.8 & 48.9 & 49.6 & 51.8 & 51.8 & 57.3 & 63.5 \\
\hline Pending bills & -0.1 & 0.0 & 3.6 & 0.0 & 0.0 & 0.0 & 0.0 & 0.0 & 0.0 \\
\hline Development and net lending & 80.3 & 135.5 & 198.3 & 183.2 & 240.7 & 271.6 & 302.7 & 319.1 & 368.3 \\
\hline Domestically financed & 53.5 & 90.3 & 113.3 & 115.2 & 150.3 & 157.6 & 178.3 & 192.5 & 216.2 \\
\hline Foreign financed & 26.1 & 42.9 & 81.2 & 65.6 & 87.8 & 111.6 & 122.0 & 124.2 & 149.7 \\
\hline Of which: financed by sovereign bonds & & 0.0 & 33.6 & 0.0 & 0.0 & 14.4 & 16.0 & 16.0 & 16.0 \\
\hline Net lending & 1.4 & 2.3 & 2.1 & 2.4 & 2.6 & 2.4 & 2.4 & 2.4 & 2.4 \\
\hline Pending bills & -0.7 & 0.0 & 1.6 & 0.0 & 0.0 & 0.0 & 0.0 & 0.0 & 0.0 \\
\hline Drought Development Expenditure & 0.0 & 0.0 & 0.0 & 0.0 & 0.0 & 0.0 & 0.0 & 0.0 & 0.0 \\
\hline Civil Contingency Fund & 0.0 & 0.0 & 2.0 & 2.0 & 2.3 & 2.5 & 2.8 & 2.8 & 2.8 \\
\hline Drought expenditures & 0.0 & 0.0 & 1.0 & 1.0 & 1.0 & 1.2 & 1.3 & 1.4 & 1.6 \\
\hline Balance (commitment basis, excluding grants) & -46.5 & -106.7 & -160.2 & -143.1 & -176.7 & -175.3 & -186.4 & -192.8 & -212.7 \\
\hline Grants & 15.5 & 25.5 & 33.8 & 25.0 & 35.6 & 41.5 & 45.8 & 42.8 & 46.4 \\
\hline Food/debt relief grants 3 / & 0.0 & 0.4 & 0.0 & 0.0 & 0.0 & 0.0 & 0.0 & 0.0 & 0.0 \\
\hline Project grants & 15.5 & 21.3 & 33.8 & 25.0 & 35.6 & 41.5 & 45.8 & 42.8 & 46.4 \\
\hline Program grants & 0.0 & 3.8 & 0.0 & 0.0 & 0.0 & 0.0 & 0.0 & 0.0 & 0.0 \\
\hline Balance (commitment basis, including grants) & -31.0 & -81.2 & -126.4 & -118.1 & -141.1 & -133.8 & -140.6 & -150.0 & -166.3 \\
\hline Adjustments to cash basis & 1.6 & 9.3 & -0.4 & -0.4 & 0.0 & 0.0 & 0.0 & 0.0 & 0.0 \\
\hline Balance (cash basis, including grants) & -29.4 & -71.9 & -126.9 & -118.5 & -141.1 & -133.8 & -140.6 & -150.0 & -166.3 \\
\hline Financing & 35.5 & 59.8 & 126.9 & 118.5 & 141.1 & 133.8 & 140.6 & 150.0 & 166.3 \\
\hline Net foreign financing & -3.1 & 6.3 & 58.8 & 23.6 & 26.1 & 47.6 & 52.5 & 55.8 & 87.4 \\
\hline Project loans & 10.6 & 21.2 & 47.4 & 40.6 & 52.2 & 55.7 & 60.2 & 65.4 & 87.3 \\
\hline Program loans & 0.0 & 1.3 & 0.0 & 0.0 & 0.0 & 0.0 & 0.0 & 0.0 & 0.0 \\
\hline Commercial borrowing 4/ & 0.0 & 0.0 & 33.6 & 0.0 & 0.0 & 14.4 & 16.0 & 16.0 & 16.0 \\
\hline Repayments due & -16.7 & -16.5 & -16.6 & -17.5 & -20.9 & -22.5 & -23.7 & -25.6 & -15.8 \\
\hline Change in arrears & 0.7 & 0.3 & -6.1 & 0.0 & -5.2 & 0.0 & 0.0 & 0.0 & 0.0 \\
\hline Rescheduling / debt swap & 2.2 & 0.0 & 0.5 & 0.5 & 0.0 & 0.0 & 0.0 & 0.0 & 0.0 \\
\hline Privatization proceeds and other $5 /$ & 4.0 & 76.3 & 13.7 & 0.0 & 6.0 & 7.5 & 7.7 & 2.0 & 0.0 \\
\hline Bank restructuring costs $6 /$ & -20.0 & -1.1 & 0.0 & 0.0 & 0.0 & 0.0 & 0.0 & 0.0 & 0.0 \\
\hline Expenditure arrears securitization costs & 0.0 & -0.1 & -0.1 & 0.0 & 0.0 & 0.0 & 0.0 & 0.0 & 0.0 \\
\hline Telkom restructuring costs (cash) $7 /$ & $\ldots$ & -8.8 & 0.0 & 0.0 & 0.0 & 0.0 & 0.0 & 0.0 & 0.0 \\
\hline Bank restructuring financing $6 /$ & 20.0 & 1.1 & 0.0 & 0.0 & 0.0 & 0.0 & 0.0 & 0.0 & 0.0 \\
\hline Telkom/KPRL refinancing & & & $\ldots$ & 2.5 & $\ldots$ & & & $\ldots$ & \\
\hline Net domestic financing & 34.7 & -13.9 & 54.5 & 92.3 & 109.0 & 78.7 & 80.4 & 92.2 & 78.9 \\
\hline Financing gap (stat. discrepancy for outturns) & -6.2 & 12.1 & 0.0 & 0.0 & 0.0 & 0.0 & 0.0 & 0.0 & 0.0 \\
\hline Memorandum items: & & & & & & & & & \\
\hline Nominal GDP & $1,717.5$ & $1,986.0$ & $2,393.2$ & $2,294.1$ & $2,604.6$ & $2,900.5$ & $3,218.1$ & $3,594.3$ & $4,017.0$ \\
\hline Primary budget balance & 13.2 & -24.0 & -70.2 & -64.5 & -75.0 & -59.1 & -56.9 & -54.0 & -64.0 \\
\hline Stock of domestic debt, net (end of period) & 332.3 & 332.1 & 386.6 & 424.4 & 533.5 & 612.2 & 692.6 & 784.8 & 863.7 \\
\hline NPV of total public debt, net & 613.7 & 564.7 & $\ldots$ & 677.1 & 840.5 & 930.0 & $1,041.3$ & $1,186.2$ & $1,325.4$ \\
\hline Total public debt, net & 723.4 & 729.8 & $\ldots$ & 947.7 & $1,103.2$ & $1,182.2$ & $1,317.8$ & $1,502.6$ & $1,674.5$ \\
\hline
\end{tabular}

Sources: Kenyan authorities; and IMF staff estimates and projections.

$1 /$ Fiscal year runs from July to June.

2/ Includes a one-time allocation for payment of security-related arrears of Ksh 2 billion in 2007/08 budget.

3/ Includes debt relief from a debt swap deal with Italy.

4/ Includes planned sovereign bonds.

5/ In 2008/09, this includes repayment from parastatals of expenditures that are pre-financed during 2008/09 by the central government budget.

6/ Operation consists of recapitalization of National Bank of Kenya and financing this through issuance of a special purpose bond.

7/ Operation consists of recapitalization of Kenya Telkom on account of its pension obligations and restructuring operation and financing this through cash injection. 
Table 2b. Kenya: Central Government Financial Operations, 2006/07-2013/14 ${ }^{1 /}$

\begin{tabular}{|c|c|c|c|c|c|c|c|c|c|}
\hline & \multirow{2}{*}{$\begin{array}{r}2006 / 07 \\
\text { Actual } \\
\end{array}$} & \multirow{2}{*}{$\begin{array}{l}2007 / 08 \\
\text { Estimate } \\
\end{array}$} & \multicolumn{2}{|c|}{$2008 / 09$} & \multirow{2}{*}{$\begin{array}{c}2009 / 10 \\
\text { Staff } \\
\text { Projection }\end{array}$} & \multirow[t]{2}{*}{$\underline{2010 / 11}$} & $2011 / 12$ & \multirow{2}{*}{$\begin{array}{l}2012 / 13 \\
\text { tions }\end{array}$} & \multirow[t]{2}{*}{$2013 / 14$} \\
\hline & & & Budget & $\begin{array}{c}\text { Staff } \\
\text { Projection }\end{array}$ & & & Projec & & \\
\hline & \multicolumn{9}{|c|}{ (In percent of GDP, unless otherwise indicated) } \\
\hline Revenue & 21.7 & 21.8 & 21.4 & 22.3 & 21.7 & 21.7 & 21.7 & 21.7 & 21.6 \\
\hline Income tax & 7.7 & 8.3 & 8.1 & 8.4 & 8.4 & 8.4 & 8.4 & 8.4 & 8.4 \\
\hline Import duty (net) & 1.6 & 1.7 & 1.5 & 1.6 & 1.6 & 1.5 & 1.5 & 1.4 & 1.4 \\
\hline Excise duty & 3.3 & 3.1 & 3.0 & 3.0 & 2.9 & 2.9 & 2.9 & 2.9 & 2.9 \\
\hline Value-added tax & 5.6 & 5.6 & 5.6 & 5.6 & 5.4 & 5.5 & 5.5 & 5.5 & 5.5 \\
\hline Investment income & 0.4 & 0.2 & 0.2 & 0.3 & 0.3 & 0.3 & 0.3 & 0.3 & 0.3 \\
\hline Other & 1.7 & 1.5 & 1.4 & 1.7 & 1.6 & 1.6 & 1.6 & 1.6 & 1.6 \\
\hline Ministerial and Departmental Fees (AIA) & 1.5 & 1.4 & 1.5 & 1.6 & 1.6 & 1.6 & 1.6 & 1.6 & 1.6 \\
\hline Expenditure and net lending & 24.4 & 27.1 & 28.1 & 28.5 & 28.5 & 27.7 & 27.5 & 27.1 & 26.9 \\
\hline Recurrent expenditure & 19.8 & 20.3 & 19.7 & 20.4 & 19.1 & 18.3 & 18.0 & 18.1 & 17.7 \\
\hline Interest payments & 2.5 & 2.4 & 2.4 & 2.4 & 2.5 & 2.6 & 2.6 & 2.7 & 2.5 \\
\hline Domestic interest & 2.1 & 2.1 & 2.1 & 2.1 & 2.2 & 2.3 & 2.3 & 2.3 & 2.2 \\
\hline Foreign interest due & 0.3 & 0.3 & 0.3 & 0.3 & 0.3 & 0.3 & 0.3 & 0.4 & 0.3 \\
\hline Wages and benefits (civil service) & 7.4 & 7.4 & 6.8 & 6.9 & 6.7 & 6.5 & 6.3 & 6.3 & 6.3 \\
\hline Civil service reform & 0.1 & 0.0 & 0.0 & 0.0 & 0.0 & 0.0 & 0.0 & 0.0 & 0.0 \\
\hline Pensions, etc. & 1.2 & 1.2 & 1.1 & 1.1 & 1.2 & 1.2 & 1.2 & 1.2 & 1.2 \\
\hline Other & 6.9 & 7.0 & 7.2 & 7.8 & 6.8 & 6.2 & 6.2 & 6.3 & 6.0 \\
\hline Defense and NSIS 2/ & 1.7 & 2.3 & 2.1 & 2.1 & 1.9 & 1.8 & 1.6 & 1.6 & 1.6 \\
\hline Pending bills & 0.0 & 0.0 & 0.2 & 0.0 & 0.0 & 0.0 & 0.0 & 0.0 & 0.0 \\
\hline Development and net lending & 4.7 & 6.8 & 8.3 & 8.0 & 9.2 & 9.4 & 9.4 & 8.9 & 9.2 \\
\hline Domestically financed & 3.1 & 4.5 & 4.7 & 5.0 & 5.8 & 5.4 & 5.5 & 5.4 & 5.4 \\
\hline Foreign financed & 1.5 & 2.2 & 3.4 & 2.9 & 3.4 & 3.8 & 3.8 & 3.5 & 3.7 \\
\hline Of which: financed by sovereign bonds & & 0.0 & 1.4 & 0.0 & 0.0 & 0.5 & 0.5 & 0.4 & 0.4 \\
\hline Net lending & 0.1 & 0.1 & 0.1 & 0.1 & 0.1 & 0.1 & 0.1 & 0.1 & 0.1 \\
\hline Pending bills & 0.0 & 0.0 & 0.1 & 0.0 & 0.0 & 0.0 & 0.0 & 0.0 & 0.0 \\
\hline Drought Development Expenditure & 0.0 & 0.0 & 0.0 & 0.0 & 0.0 & 0.0 & 0.0 & 0.0 & 0.0 \\
\hline Civil Contingency Fund & 0.0 & 0.0 & 0.1 & 0.1 & 0.1 & 0.1 & 0.1 & 0.1 & 0.1 \\
\hline Drought expenditures & 0.0 & 0.0 & 0.0 & 0.0 & 0.0 & 0.0 & 0.0 & 0.0 & 0.0 \\
\hline Balance (commitment basis, excluding grants) & -2.7 & -5.4 & -6.7 & -6.2 & -6.8 & -6.0 & -5.8 & -5.4 & -5.3 \\
\hline Grants & 0.9 & 1.3 & 1.4 & 1.1 & 1.4 & 1.4 & 1.4 & 1.2 & 1.2 \\
\hline Food/debt relief grants 3 / & 0.0 & 0.0 & 0.0 & 0.0 & 0.0 & 0.0 & 0.0 & 0.0 & 0.0 \\
\hline Project grants & 0.9 & 1.1 & 1.4 & 1.1 & 1.4 & 1.4 & 1.4 & 1.2 & 1.2 \\
\hline Program grants & 0.0 & 0.2 & 0.0 & 0.0 & 0.0 & 0.0 & 0.0 & 0.0 & 0.0 \\
\hline Balance (commitment basis, including grants) & -1.8 & -4.1 & -5.3 & -5.1 & -5.4 & -4.6 & -4.4 & -4.2 & -4.1 \\
\hline Adjustments to cash basis & 0.1 & 0.5 & 0.0 & 0.0 & 0.0 & 0.0 & 0.0 & 0.0 & 0.0 \\
\hline Balance (cash basis, including grants) & -1.7 & -3.6 & -5.3 & -5.2 & -5.4 & -4.6 & -4.4 & -4.2 & -4.1 \\
\hline Financing & 2.1 & 3.0 & 5.3 & 5.2 & 5.4 & 4.6 & 4.4 & 4.2 & 4.1 \\
\hline Net foreign financing & -0.2 & 0.3 & 2.5 & 1.0 & 1.0 & 1.6 & 1.6 & 1.6 & 2.2 \\
\hline Project loans & 0.6 & 1.1 & 2.0 & 1.8 & 2.0 & 1.9 & 1.9 & 1.8 & 2.2 \\
\hline Program loans & 0.0 & 0.1 & 0.0 & 0.0 & 0 & 0.0 & 0.0 & 0.0 & 0.0 \\
\hline Commercial borrowing 4/ & 0.0 & 0.0 & 1.4 & 0.0 & 0.0 & 0.5 & 0.5 & 0.4 & 0.4 \\
\hline Repayments due & -1.0 & -0.8 & -0.7 & -0.8 & -0.8 & -0.8 & -0.7 & -0.7 & -0.4 \\
\hline Change in arrears & 0.0 & 0.0 & -0.3 & 0.0 & -0.2 & 0.0 & 0.0 & 0.0 & 0.0 \\
\hline Rescheduling / debt swap & 0.1 & 0.0 & 0.0 & 0.0 & 0.0 & 0.0 & 0.0 & 0.0 & 0.0 \\
\hline Privatization proceeds and other $5 /$ & 0.2 & 3.8 & 0.6 & 0.0 & 0.2 & 0.3 & 0.2 & 0.1 & 0.0 \\
\hline Bank restructuring costs 6 / & -1.2 & -0.1 & 0.0 & 0.0 & 0.0 & 0.0 & 0.0 & 0.0 & 0.0 \\
\hline Expenditure arrears securitization costs & 0.0 & 0.0 & 0.0 & 0.0 & 0.0 & 0.0 & 0.0 & 0.0 & 0.0 \\
\hline Telkom restructuring costs (cash) $7 /$ & & -0.4 & 0.0 & 0.0 & 0.0 & 0.0 & 0.0 & 0.0 & 0.0 \\
\hline Bank restructuring financing $6 /$ & 1.2 & 0.1 & 0.0 & 0.0 & 0.0 & 0.0 & 0.0 & 0.0 & 0.0 \\
\hline Telkom/KPRL refinancing & & & & 0.1 & & & & & \\
\hline Net domestic borrowing & 2.0 & -0.7 & 2.3 & 4.0 & 4.2 & 2.7 & 2.5 & 2.6 & 2.0 \\
\hline Financing gap (stat. discrepancy for outturns) & -0.4 & 0.6 & 0.0 & 0.0 & 0.0 & 0.0 & 0.0 & 0.0 & 0.0 \\
\hline \multicolumn{10}{|l|}{ Memorandum items: } \\
\hline Nominal GDP (billion of Ksh) & $1,717.5$ & $1,986.0$ & $2,393.2$ & $2,294.1$ & $2,604.6$ & $2,900.5$ & $3,218.1$ & $3,594.3$ & $4,017.0$ \\
\hline Primary budget balance & 0.8 & -1.2 & -2.9 & -2.8 & -2.9 & -2.0 & -1.8 & -1.5 & -1.6 \\
\hline Stock of domestic debt, net (end of period) & 19.3 & 16.7 & 16.2 & 18.5 & 20.5 & 21.1 & 21.5 & 21.8 & 21.5 \\
\hline Total public debt, net & 42.1 & 36.7 & $\ldots$ & 41.3 & 42.4 & 40.8 & 40.9 & 41.8 & 41.7 \\
\hline
\end{tabular}

Sources: Kenyan authorities; and IMF staff estimates and projections.

1/ Fiscal year runs from July to June.

2/ Includes a one-time allocation for payment of security-related arrears of Ksh 2 billion in 2007/08 budget.

3 / Includes debt relief from a debt swap deal with Italy.

$4 /$ Includes planned sovereign bonds.

5/ In 2008/09, this includes repayment from parastatals of expenditures that are pre-financed during 2008/09 by the central government budget.

6/ Operation consists of recapitalization of National Bank of Kenya and financing this through issuance of a special purpose bond.

7/ Operation consists of recapitalization of Kenya Telkom on account of its pension obligations and restructuring operation and financing this through cash injection.

CInternational Monetary Fund. Not for Redistribution 
Table 3. Kenya: Monetary Survey, 2006-10

\begin{tabular}{|c|c|c|c|c|c|c|c|c|c|c|c|}
\hline & Jun-06 & Jun-07 & Jun-08 & Sep-08 & Dec-08 & Mar-09 & Jun-09 & Sep-09 & Dec-09 & Mar-10 & Jun-10 \\
\hline & & & & & & & \multicolumn{5}{|c|}{ Projections } \\
\hline & \multicolumn{11}{|c|}{ (Billions of Kenyan shillings) } \\
\hline Central Bank of Kenya (CBK) & & & & & & & & & & & \\
\hline Net Foreign assets & 158.2 & 163.7 & 202.6 & 208.7 & 199.1 & 193.4 & 206.4 & 218.3 & 229.1 & 218.9 & 215.8 \\
\hline In millions of US\$ & 2148.7 & 2461.1 & 3145.2 & 2855.2 & 2566.1 & 2424.4 & 2580.0 & 2630.1 & 2705.1 & 2736.3 & 2766.7 \\
\hline Net domestic assets & -50.3 & -34.3 & -49.6 & -56.1 & -35.5 & -37.9 & -41.9 & -49.6 & -44.6 & -40.7 & -28.6 \\
\hline Net domestic credit & -37.0 & -16.8 & -20.7 & -12.7 & -1.5 & -6.2 & -4.1 & -10.8 & -2.2 & 0.2 & 14.5 \\
\hline Government (net) & -15.8 & -3.5 & -27.7 & -15.1 & 0.0 & 0.9 & 3.4 & 4.7 & 6.1 & 7.5 & 8.8 \\
\hline Commercial banks (net) & -23.4 & -15.7 & 4.5 & 0.0 & -4.0 & -9.7 & -10.0 & -18.1 & -10.8 & -9.8 & 3.1 \\
\hline Other items (net) & -13.2 & -17.5 & -28.9 & -43.4 & -34.0 & -31.6 & -37.8 & -38.8 & -42.4 & -41.0 & -43.1 \\
\hline Reserve Money & 108.0 & 129.4 & 152.9 & 152.6 & 163.6 & 155.5 & 164.5 & 168.7 & 184.5 & 178.2 & 187.2 \\
\hline Currency outside banks & 67.2 & 78.1 & 83.6 & 85.4 & 93.7 & 88.0 & 98.7 & 100.7 & 107.3 & 107.1 & 114.3 \\
\hline Banks reserves & 40.8 & 51.2 & 69.3 & 67.2 & 69.9 & 67.5 & 65.7 & 67.9 & 77.2 & 71.1 & 72.9 \\
\hline \multicolumn{12}{|l|}{ Other Depository Corporation Survey } \\
\hline Net foreign assets & 32.0 & 59.1 & 88.7 & 53.9 & 59.9 & 72.9 & 85.5 & 91.6 & 99.2 & 98.0 & 99.0 \\
\hline In millions of US\$ & 434.6 & 888.5 & 1377.8 & 736.8 & 771.3 & 913.9 & 1068.7 & 1103.9 & 1171.6 & 1225.4 & 1269.3 \\
\hline Reserves & 40.8 & 51.2 & 69.3 & 67.2 & 69.9 & 67.5 & 65.7 & 67.9 & 77.2 & 71.1 & 72.9 \\
\hline Credit to CBK & 23.4 & 15.7 & -4.5 & 0.0 & 4.0 & 9.7 & 10.0 & 18.1 & 10.8 & 9.8 & -3.1 \\
\hline Net domestic assets & 441.9 & 504.3 & 603.6 & 644.2 & 664.2 & 659.7 & 690.0 & 690.7 & 737.6 & 744.2 & 816.9 \\
\hline Domestic credit & 535.1 & 601.5 & 727.0 & 788.7 & 806.0 & 826.1 & 856.0 & 874.9 & 933.7 & 950.2 & 1036.9 \\
\hline Government (net) & 133.7 & 160.6 & 161.1 & 165.1 & 155.3 & 167.2 & 186.9 & 189.6 & 192.3 & 195.0 & 205.9 \\
\hline Other public sector & 12.2 & 12.5 & 10.1 & 12.2 & 11.8 & 7.4 & 10.4 & 12.6 & 12.2 & 7.6 & 10.7 \\
\hline Privates sector & 389.3 & 428.4 & 555.8 & 611.4 & 638.9 & 651.5 & 658.7 & 672.7 & 729.2 & 747.5 & 820.3 \\
\hline Other Items (Net) & -93.3 & -97.2 & -123.4 & -144.5 & -141.8 & -166.4 & -165.9 & -184.1 & -196.1 & -206.0 & -220.0 \\
\hline Total deposits & 538.0 & 630.2 & 757.1 & 765.3 & 797.9 & 809.7 & 851.2 & 868.4 & 924.8 & 923.1 & 985.7 \\
\hline \multicolumn{12}{|l|}{ Depository Corporation Survey } \\
\hline Net Foreign assets & 190.2 & 222.7 & 291.3 & 262.6 & 259.0 & 266.3 & 291.9 & 309.9 & 328.3 & 316.9 & 314.8 \\
\hline In millions of US\$ & 2583.3 & 3349.6 & 4522.9 & 3592.0 & 3337.4 & 3338.3 & 3648.7 & 3734.0 & 3876.7 & 3961.7 & 4036.0 \\
\hline Net domestic assets & 415.0 & 485.6 & 549.4 & 596.8 & 642.1 & 639.8 & 658.1 & 659.2 & 703.8 & 713.2 & 785.3 \\
\hline Domestic credit & 521.5 & 600.3 & 701.8 & 776.0 & 808.5 & 829.5 & 861.8 & 882.1 & 942.3 & 960.2 & 1048.3 \\
\hline Government (Net) & 117.9 & 157.2 & 133.4 & 149.9 & 155.3 & 168.1 & 190.2 & 194.3 & 198.4 & 202.5 & 214.8 \\
\hline Other Public Sector & 12.2 & 12.5 & 10.1 & 12.2 & 11.8 & 7.4 & 10.4 & 12.6 & 12.2 & 7.6 & 10.7 \\
\hline Private Sector ${ }^{\star}$ & 391.4 & 430.7 & 558.3 & 613.9 & 641.4 & 654.0 & 661.2 & 675.2 & 731.8 & 750.1 & 822.8 \\
\hline Other Items (Net ) * & -106.5 & -114.7 & -152.4 & -179.3 & -166.4 & -189.7 & -203.8 & -222.9 & -238.5 & -247.0 & -263.0 \\
\hline Money and Quasi-money (M3) & 522.0 & 605.5 & 716.0 & 736.3 & 766.4 & 780.6 & 771.8 & 790.6 & 853.3 & 850.9 & 920.5 \\
\hline M3 plus Residents' Foreign Curr. Deposits (M3X) & 605.2 & 708.4 & 840.7 & 859.3 & 901.1 & 906.1 & 950.0 & 969.1 & 1032.1 & 1030.2 & 1100.1 \\
\hline M3X plus Nonbank Holdings of Govt. Debt (M4X) & 765.6 & 884.4 & 1028.6 & 1051.2 & 1091.9 & 1101.7 & 1173.4 & 1215.7 & 1301.9 & 1323.1 & 1408.0 \\
\hline \multicolumn{12}{|l|}{ Memorandum items: } \\
\hline & \multicolumn{11}{|c|}{ (Annual percent change) } \\
\hline Money and Quasi-money (M3) & 18.0 & 16.0 & 18.2 & 16.7 & 14.9 & 12.0 & 7.8 & 7.4 & 11.3 & 9.0 & 19.3 \\
\hline M3 plus Residents' Foreign Curr. Deposits (M3X) & 15.6 & 17.0 & 18.7 & 17.2 & 15.9 & 11.7 & 13.0 & 12.8 & 14.5 & 13.7 & 15.8 \\
\hline M3X plus Nonbank Holdings of Govt. Debt (M4X) & 15.8 & 15.5 & 16.3 & 14.5 & 12.4 & 9.5 & 14.1 & 15.6 & 19.2 & 20.1 & 20.0 \\
\hline Reserve Money & 14.2 & 19.8 & 18.2 & 14.5 & 4.2 & 1.6 & 7.6 & 10.5 & 12.8 & 14.6 & 13.8 \\
\hline Currency Outside Banks & 13.3 & 16.2 & 7.0 & 6.7 & -2.3 & 3.6 & 18.1 & 17.9 & 14.4 & 21.6 & 15.8 \\
\hline NFA & 22.9 & 17.1 & 30.8 & 16.4 & 1.4 & 6.7 & 0.2 & 18.0 & 26.8 & 19.0 & 7.8 \\
\hline NDA of the banking sector & 12.5 & 17.0 & 13.1 & 17.5 & 22.9 & 13.9 & 19.8 & 10.5 & 9.6 & 11.5 & 19.3 \\
\hline Domestic credit & 12.3 & 15.1 & 16.9 & 22.9 & 23.2 & 19.8 & 22.8 & 13.7 & 16.6 & 15.8 & 21.6 \\
\hline Government (net) & 5.0 & 33.3 & -15.1 & -6.5 & 13.0 & 4.7 & 42.6 & 29.6 & 27.7 & 20.5 & 12.9 \\
\hline Credit to private sector & 14.5 & 10.0 & 29.6 & 33.9 & 27.2 & 26.7 & 18.4 & 10.0 & 14.1 & 14.7 & 24.4 \\
\hline Other Items Net & 11.4 & 7.7 & 32.8 & 45.0 & 24.3 & 45.3 & 33.7 & 24.4 & 43.3 & 30.2 & 29.1 \\
\hline Money multiplier (M3X/RM) & 5.6 & 5.5 & 5.5 & 5.6 & 5.5 & 5.8 & 5.8 & 5.7 & 5.6 & 5.8 & 5.9 \\
\hline Money Velocity (GDP/M3X) & 2.6 & 2.5 & 2.4 & 2.3 & 2.3 & 2.3 & 2.3 & 2.3 & 2.3 & 2.3 & 2.3 \\
\hline
\end{tabular}


Table 4a. Kenya: Balance of Payments, 2006/07-2013/14

(In millions of U.S. dollars, unless otherwise indicated)

\begin{tabular}{|c|c|c|c|c|c|c|c|c|}
\hline & $2006 / 07$ & 2007/08 & $2008 / 09$ & $2009 / 10$ & $2010 / 11$ & $2011 / 12$ & $2012 / 13$ & $2013 / 14$ \\
\hline & Est. & Prel. & \multicolumn{6}{|c|}{ Projections } \\
\hline Current account & -849.3 & $-1,410.4$ & $-1,769.8$ & $-1,458.3$ & $-1,870.2$ & $-2,084.1$ & $-2,090.9$ & $-2,034.5$ \\
\hline Excluding official transfers & -849.3 & $-1,465.2$ & $-1,759.4$ & $-1,436.1$ & $-1,848.0$ & $-2,061.8$ & $-2,068.7$ & $-2,012.2$ \\
\hline Exports, f.o.b. & $3,834.8$ & $4,506.6$ & $4,789.3$ & $4,782.9$ & $5,303.1$ & $5,958.0$ & $6,813.7$ & $7,880.9$ \\
\hline Coffee & 142.5 & 169.3 & 132.8 & 118.0 & 128.1 & 139.7 & 156.8 & 178.0 \\
\hline Tea & 726.8 & 754.8 & 874.5 & 702.9 & 724.7 & 793.2 & 889.8 & $1,021.4$ \\
\hline Horticulture & 552.0 & 739.3 & 644.6 & 652.5 & 746.3 & 856.4 & 999.3 & $1,191.2$ \\
\hline Imports, f.o.b. & $-7,777.9$ & $-9,212.3$ & $-9,701.4$ & $-9,181.1$ & $-10,359.4$ & $-11,585.0$ & $-12,856.8$ & $-14,360.3$ \\
\hline Oil & $-1,755.1$ & $-2,448.0$ & $-2,485.9$ & $-1,922.8$ & $-2,303.7$ & $-2,629.5$ & $-2,866.9$ & $-3,115.7$ \\
\hline Other & $-5,902.7$ & $-6,739.2$ & $-7,108.5$ & $-7,162.2$ & $-7,955.8$ & $-8,851.9$ & $-9,882.4$ & $-11,132.9$ \\
\hline of which: Special: Maize \& sugar & -132.3 & -100.0 & -257.4 & -224.8 & -164.5 & -176.6 & -185.9 & -191.7 \\
\hline Balance on goods & $-3,943.1$ & $-4,705.7$ & $-4,912.1$ & $-4,398.2$ & $-5,056.3$ & $-5,627.0$ & $-6,043.1$ & $-6,479.4$ \\
\hline Services (net) & $1,568.6$ & $1,455.1$ & $1,219.5$ & $1,064.6$ & $1,222.4$ & $1,445.7$ & $1,682.6$ & $1,936.2$ \\
\hline Credit & $2,611.6$ & $3,037.4$ & $3,052.6$ & $2,852.2$ & $3,154.2$ & $3,527.5$ & $3,936.4$ & $4,383.8$ \\
\hline of which: foreign travel credit ${ }^{\perp \prime}$ & 731.6 & 686.1 & 649.9 & 606.8 & 713.4 & 856.1 & $1,000.2$ & $1,134.7$ \\
\hline Debit & $-1,043.0$ & $-1,582.3$ & $-1,833.1$ & $-1,787.6$ & $-1,931.8$ & $-2,081.9$ & $-2,253.7$ & $-2,447.6$ \\
\hline Balance on goods and services & $-2,374.5$ & $-3,250.6$ & $-3,692.6$ & $-3,333.6$ & $-3,833.8$ & $-4,181.3$ & $-4,360.5$ & $-4,543.2$ \\
\hline Income (net) & -84.3 & -45.6 & -29.8 & -40.5 & -61.6 & -77.1 & -96.5 & -96.5 \\
\hline Credit & 86.2 & 149.1 & 120.2 & 116.5 & 110.9 & 122.8 & 137.1 & 154.3 \\
\hline Debit & -170.5 & -194.7 & -150.0 & -157.1 & -172.5 & -199.9 & -233.6 & -250.8 \\
\hline of which : ofticial interest payments & -97.0 & -85.7 & -86.5 & -97.1 & -115.7 & -145.5 & -180.3 & -197.1 \\
\hline Other & -73.4 & -109.0 & -63.6 & -60.0 & -56.9 & -54.4 & -53.3 & -53.6 \\
\hline Current transfers (net) & $1,609.5$ & $1,885.8$ & $1,952.7$ & $1,915.7$ & $2,025.2$ & $2,174.3$ & $2,366.1$ & $2,605.2$ \\
\hline Private (net) & $1,609.5$ & $1,831.0$ & $1,963.1$ & $1,938.0$ & $2,047.5$ & $2,196.6$ & $2,388.3$ & $2,627.5$ \\
\hline of which: remittances & 816.1 & 952.8 & 933.8 & 906.8 & 963.8 & $1,036.0$ & $1,127.0$ & $1,239.2$ \\
\hline Official (net) & 0.0 & 54.8 & -10.4 & -22.3 & -22.3 & -22.3 & -22.3 & -22.3 \\
\hline Capital and financial account & $1,036.7$ & $2,101.4$ & 974.5 & $1,690.3$ & $2,266.5$ & $2,603.1$ & $2,699.9$ & $2,662.0$ \\
\hline Capital account (incl. capital transfers) & 223.9 & 325.9 & 319.7 & 440.7 & 562.9 & 635.6 & 587.1 & 627.8 \\
\hline Financial account 2/ & 812.8 & $1,775.5$ & 654.8 & $1,249.6$ & $1,703.6$ & $1,967.5$ & $2,112.8$ & $2,034.2$ \\
\hline Net FDI & 622.7 & 891.7 & 658.1 & 466.5 & 534.5 & 644.2 & 703.3 & 767.5 \\
\hline In Kenya & 682.5 & 994.4 & 677.6 & 490.5 & 558.5 & 668.2 & 727.3 & 791.5 \\
\hline Abroad & -59.8 & -102.7 & -19.5 & -24.0 & -24.0 & -24.0 & -24.0 & -24.0 \\
\hline Net Portfolio investment & -22.6 & -36.5 & -10.3 & -2.2 & 3.4 & 7.4 & 8.5 & 9.6 \\
\hline Liabilities & 1.2 & 6.1 & 8.9 & 10.4 & 12.9 & 16.6 & 17.8 & 19.2 \\
\hline Assets & -23.9 & -42.6 & -19.2 & -12.7 & -9.5 & -9.2 & -9.4 & -9.6 \\
\hline Net other investment & 212.8 & 920.3 & 7.0 & 785.3 & $1,165.8$ & $1,315.8$ & $1,401.1$ & $1,257.1$ \\
\hline Official, medium and long term & -5.1 & 163.4 & 331.3 & 464.6 & 723.6 & 798.4 & 763.7 & 976.8 \\
\hline Inflows & 246.4 & 428.4 & 573.2 & 718.8 & $1,010.0$ & $1,092.4$ & $1,135.4$ & $1,403.3$ \\
\hline Program loans & 0.0 & 20.0 & 0.0 & 0.0 & 0.0 & 0.0 & 0.0 & 0.0 \\
\hline Project loans & 153.3 & 325.0 & 515.1 & 662.5 & 753.7 & 836.1 & 896.0 & $1,180.8$ \\
\hline Commercial loans & 20.9 & 0.0 & 0.0 & 0.0 & 200.0 & 200.0 & 200.0 & 200.0 \\
\hline Government guaranteed/parastatal & 72.2 & 83.5 & 58.1 & 56.3 & 56.3 & 56.3 & 39.4 & 22.5 \\
\hline Outflows & -251.4 & -265.1 & -241.9 & -254.2 & -286.4 & -294.0 & -371.7 & -426.5 \\
\hline Private, medium and long term & -18.1 & 40.4 & 23.9 & 15.9 & 40.0 & 107.4 & 97.7 & 47.9 \\
\hline Energy financing & 19.3 & 48.7 & 58.4 & 54.0 & 60.0 & 60.0 & 60.0 & 42.1 \\
\hline Kenya Airways & 33.1 & 27.1 & -47.6 & -50.9 & 25.2 & 97.0 & 88.3 & 59.6 \\
\hline Other & -70.4 & -35.4 & 13.1 & 12.8 & -45.2 & -49.6 & -50.5 & -53.8 \\
\hline \multirow{2}{*}{$\begin{array}{l}\text { Short-term capital and errors and omissions } 2 / \\
\text { of which: commercial banks }\end{array}$} & 235.9 & 716.5 & -348.2 & 304.8 & 402.1 & 410.1 & 539.6 & 232.4 \\
\hline & -453.9 & -485.1 & 304.8 & -200.6 & -158.6 & -227.0 & -186.7 & -179.0 \\
\hline Overall balance & 187.4 & 691.1 & -795.3 & 232.0 & 396.3 & 519.0 & 609.0 & 627.5 \\
\hline Financing items & -187.4 & -691.1 & 795.3 & -232.0 & -396.3 & -519.0 & -609.0 & -627.5 \\
\hline Reserve assets (gross) & -370.2 & -719.8 & 647.1 & -203.5 & -369.3 & -497.0 & -570.3 & -605.3 \\
\hline Use of Fund credit and loans to the Fund (net) & 59.3 & 54.8 & -8.7 & -17.3 & -27.0 & -22.0 & -38.7 & -22.2 \\
\hline Change in arrears $3 /$ & 69.9 & -26.0 & 0.0 & -61.4 & 0.0 & 0.0 & 0.0 & 0.0 \\
\hline Rescheduling /debt swap & 53.6 & 0.0 & 6.1 & 0.0 & 0.0 & 0.0 & 0.0 & 0.0 \\
\hline Remaining gap & 0.0 & 0.0 & 150.8 & 50.3 & 0.0 & 0.0 & 0.0 & 0.0 \\
\hline \multicolumn{9}{|l|}{ Memorandum items: } \\
\hline Gross official reserves (end of period) & $2,723.2$ & $3,443.0$ & $2,795.9$ & $2,999.4$ & $3,368.8$ & $3,865.7$ & $4,436.0$ & $5,041.4$ \\
\hline (in months of following year's imports of goods and $\mathrm{s}$ & 3.0 & 3.6 & 3.1 & 2.9 & 3.0 & 3.1 & 3.2 & 3.2 \\
\hline Current account balance (excl. official transfers, percent of GDP) & -3.5 & -4.8 & -5.9 & -4.5 & -4.7 & -4.6 & -4.2 & -3.7 \\
\hline Current account balance (including official transfers, percent of GDP) & -3.5 & -4.6 & -6.0 & -4.5 & -4.8 & -4.7 & -4.2 & -3.7 \\
\hline Change in reserves due to valuation ("-" represents loss) & 104.2 & 80.8 & $\ldots$ & $\ldots$ & & $\ldots$ & $\ldots$ & \\
\hline Import volume growth, goods (percent) & 10.3 & 7.5 & 5.8 & 6.8 & 7.8 & 8.5 & 8.1 & 8.5 \\
\hline Import value growth, goods (percent) & 23.6 & 18.4 & 5.3 & -5.4 & 12.8 & 11.8 & 11.0 & 11.7 \\
\hline Export volume growth, goods (percent) & 3.9 & 10.7 & 8.4 & 7.1 & 9.0 & 10.5 & 10.6 & 11.3 \\
\hline Export value growth, goods (percent) & 9.3 & 17.5 & 6.3 & -0.1 & 10.9 & 12.3 & 14.4 & 15.7 \\
\hline Change in the terms of trade (goods, percent) & -5.8 & -5.1 & 9.5 & 1.2 & -3.1 & -1.4 & 0.5 & 1.0 \\
\hline Public and publicly guaranteed external debt (percent of GDP) & 22.8 & 20.0 & 22.8 & 21.9 & 19.7 & 19.4 & 20.0 & 20.2 \\
\hline
\end{tabular}

Sources: Kenyan authorities; and IMF staff estimates and projections.

1/ The foreign travel credit is comprised of two components, recorded tourism inflows and an estimate of additional under-reported tourism receipts.

2/ Historical figures include errors and omissions.

3/ Starting in 2008/09, change in arrears reflects the amounts in the budget. For earlier years, there are differences with the fiscal sector tables (Tables $2 \mathrm{a}$ and $2 \mathrm{~b}$ ) because the government's budget presentation does not show changes to arrears associated with disputed security-related contracts. 
Table 4b. Kenya: Balance of Payments, 2006-14

\begin{tabular}{|c|c|c|c|c|c|c|c|c|c|}
\hline & 2006 & 2007 & 2008 & 2009 & 2010 & 2011 & 2012 & 2013 & 2014 \\
\hline & & & Est. & \multicolumn{6}{|c|}{ Projections } \\
\hline Current account & -563.1 & $-1,106.4$ & $-2,018.6$ & $-1,097.9$ & $-1,755.5$ & $-2,002.4$ & $-2,181.4$ & $-2,028.6$ & $-2,074.8$ \\
\hline Excluding official transfers & -625.6 & $-1,161.3$ & $-2,018.6$ & $-1,075.7$ & $-1,733.3$ & $-1,980.2$ & $-2,159.2$ & $-2,006.4$ & $-2,052.6$ \\
\hline Exports, f.o.b. & $3,516.0$ & $4,123.0$ & $4,972.2$ & $4,599.1$ & $4,970.7$ & $5,631.4$ & $6,281.2$ & $7,339.7$ & $8,412.4$ \\
\hline Coffee & 138.3 & 166.4 & 155.2 & 114.6 & 120.3 & 133.4 & 143.9 & 165.6 & 186.4 \\
\hline Tea & 654.9 & 692.6 & 923.8 & 719.9 & 688.5 & 755.5 & 825.2 & 944.5 & $1,086.5$ \\
\hline Horticulture & 508.2 & 607.5 & 763.3 & 615.7 & 687.6 & 802.3 & 907.9 & $1,086.3$ & $1,291.0$ \\
\hline Imports, f.o.b. & $-6,926.6$ & $-8,550.2$ & $-10,267.3$ & $-8,636.2$ & $-9,741.6$ & $-10,998.9$ & $-12,193.1$ & $-13,551.2$ & $-15,203.1$ \\
\hline Oil & $-1,745.3$ & $-1,919.5$ & $-3,051.2$ & $-1,716.9$ & $-2,125.5$ & $-2,479.1$ & $-2,777.3$ & $-2,955.0$ & $-3,273.9$ \\
\hline Other & $-5,032.4$ & $-6,594.7$ & $-7,132.3$ & $-6,825.1$ & $-7,518.2$ & $-8,417.9$ & $-9,310.1$ & $-10,486.5$ & $-11,815.4$ \\
\hline of which: Special: Maize \& sugar & -96.3 & -137.4 & -101.1 & -275.3 & -158.8 & -171.8 & -183.0 & -189.7 & -194.2 \\
\hline Balance on goods & $-3,410.5$ & $-4,427.1$ & $-5,295.1$ & $-4,037.1$ & $-4,770.9$ & $-5,367.4$ & $-5,911.9$ & $-6,211.5$ & $-6,790.7$ \\
\hline Services (net) & $1,322.0$ & $1,708.6$ & $1,384.9$ & $1,013.9$ & $1,122.9$ & $1,333.0$ & $1,571.0$ & $1,807.9$ & $2,078.5$ \\
\hline Credit & $2,308.6$ & $2,940.6$ & $3,255.8$ & $2,731.7$ & $2,982.6$ & $3,339.7$ & $3,730.6$ & $4,158.7$ & $4,627.0$ \\
\hline of which: foreign travel credit ${ }^{\perp \prime}$ & 628.6 & 831.4 & 673.3 & 566.1 & 651.0 & 781.2 & 937.5 & $1,068.4$ & $1,206.8$ \\
\hline Debit & -986.6 & $-1,232.0$ & $-1,870.9$ & $-1,717.8$ & $-1,859.7$ & $-2,006.8$ & $-2,159.6$ & $-2,350.8$ & $-2,548.5$ \\
\hline Balance on goods and services & $-2,088.6$ & $-2,718.5$ & $-3,910.2$ & $-3,023.2$ & $-3,648.0$ & $-4,034.4$ & $-4,340.9$ & $-4,403.7$ & $-4,712.2$ \\
\hline Income (net) & -59.3 & -106.9 & -11.4 & -21.8 & -59.4 & -63.5 & -90.4 & -102.4 & -90.3 \\
\hline Credit & 84.1 & 108.8 & 141.3 & 127.2 & 105.5 & 116.5 & 129.3 & 145.2 & 163.6 \\
\hline Debit & -143.4 & -215.8 & -152.7 & -149.0 & -164.9 & -180.0 & -219.7 & -247.6 & -253.9 \\
\hline of which : official interest payments & -108.2 & -85.9 & -85.5 & -87.4 & -106.7 & -124.6 & -166.4 & -194.2 & -200.1 \\
\hline Other & -44.7 & -129.9 & -67.2 & -61.6 & -58.2 & -55.4 & -53.2 & -53.4 & -53.8 \\
\hline Current transfers (net) & $1,584.8$ & $1,719.0$ & $1,903.1$ & $1,947.0$ & $1,951.8$ & $2,095.6$ & $2,249.8$ & $2,477.5$ & $2,727.7$ \\
\hline Private (net) & $1,522.3$ & $1,664.2$ & $1,903.1$ & $1,969.3$ & $1,974.1$ & $2,117.8$ & $2,272.1$ & $2,499.7$ & $2,750.0$ \\
\hline of which: remittances & 709.6 & 922.5 & 983.0 & 884.7 & 928.9 & 998.6 & $1,073.5$ & $1,180.4$ & $1,298.0$ \\
\hline Official (net) & 62.5 & 54.8 & 0.0 & -22.3 & -22.3 & -22.3 & -22.3 & -22.3 & -22.3 \\
\hline Capital and financial account & 904.0 & $1,929.9$ & $1,542.9$ & 912.6 & $2,144.5$ & $2,451.0$ & $2,741.4$ & $2,683.8$ & $2,671.2$ \\
\hline Capital account (incl. capital transfers) & 222.9 & 323.8 & 207.2 & 436.9 & 494.2 & 609.3 & 641.9 & 605.6 & 650.7 \\
\hline Financial account ${ }^{2 \prime}$ & 681.1 & $1,606.0$ & $1,335.7$ & 475.7 & $1,650.3$ & $1,841.7$ & $2,099.4$ & $2,078.2$ & $2,020.5$ \\
\hline Net FDI & 505.5 & 974.0 & 656.7 & 466.9 & 466.1 & 616.5 & 673.1 & 734.7 & 801.7 \\
\hline In Kenya & 543.0 & $1,074.8$ & 701.1 & 490.9 & 490.1 & 640.5 & 697.1 & 758.7 & 825.7 \\
\hline Abroad & -37.5 & -100.8 & -44.3 & -24.0 & -24.0 & -24.0 & -24.0 & -24.0 & -24.0 \\
\hline Net Portfolio investment & -20.6 & -23.8 & -27.1 & -6.9 & 0.4 & 7.1 & 8.1 & 9.2 & 10.4 \\
\hline Liabilities & 3.0 & 0.8 & 10.5 & 10.2 & 10.8 & 16.1 & 17.3 & 18.6 & 20.0 \\
\hline Assets & -23.6 & -24.6 & -37.6 & -17.2 & -10.4 & -9.0 & -9.2 & -9.4 & -9.7 \\
\hline Net other investment & 196.2 & 655.8 & 706.1 & 15.7 & $1,183.8$ & $1,218.1$ & $1,418.2$ & $1,334.3$ & $1,208.5$ \\
\hline Official, medium and long term & -69.3 & 177.2 & 51.7 & 326.7 & 870.1 & 789.3 & 767.1 & 866.5 & $1,004.7$ \\
\hline Inflows & 176.5 & 437.4 & 305.6 & 560.3 & $1,157.1$ & $1,058.8$ & $1,101.8$ & $1,275.2$ & $1,448.9$ \\
\hline Program loans & 0.0 & 20.0 & 0.0 & 0.0 & 0.0 & 0.0 & 0.0 & 0.0 & 0.0 \\
\hline Project loans & 118.1 & 300.1 & 245.6 & 504.1 & 900.8 & 802.6 & 845.6 & $1,052.7$ & $1,226.4$ \\
\hline Commercial loans & 20.9 & 10.4 & 0.0 & 0.0 & 200.0 & 200.0 & 200.0 & 200.0 & 200.0 \\
\hline Government guaranteed/parastatal & 37.5 & 106.9 & 60.0 & 56.3 & 56.3 & 56.3 & 56.3 & 22.5 & 22.5 \\
\hline Outflows & -245.8 & -260.2 & -253.9 & -233.6 & -287.0 & -269.5 & -334.7 & -408.6 & -444.3 \\
\hline Private, medium and long term & -128.6 & 100.1 & -4.7 & 55.7 & -29.8 & 110.1 & 104.8 & 90.6 & -1.6 \\
\hline Energy financing & 10.0 & 28.5 & 68.8 & 48.0 & 60.0 & 60.0 & 60.0 & 60.0 & 23.1 \\
\hline Kenya Airways & -29.5 & 95.7 & -41.4 & -53.9 & -47.9 & 98.3 & 95.6 & 80.9 & 32.1 \\
\hline other & -109.0 & -24.1 & -32.1 & 61.6 & -41.8 & -48.2 & -50.8 & -50.3 & -56.8 \\
\hline Short-term capital and errors and omissions $2 /$ & 394.1 & 378.5 & 659.1 & -366.7 & 343.5 & 318.6 & 546.2 & 377.1 & 205.4 \\
\hline of which: public net (includes trade credit) & 0.0 & 0.0 & 0.0 & 83.0 & -83.0 & 0.0 & 0.0 & 0.0 & 0.0 \\
\hline of which: commercial banks & -164.4 & -9.9 & 45.6 & -413.2 & -92.9 & -289.7 & -200.6 & -213.4 & -134.9 \\
\hline Overall balance & 340.9 & 823.4 & -475.7 & -185.4 & 389.0 & 448.6 & 560.0 & 655.2 & 596.4 \\
\hline Financing items & -340.9 & -823.4 & 475.7 & 185.4 & -389.0 & -448.6 & -560.0 & -655.2 & -596.4 \\
\hline Reserve assets (gross) & -595.3 & -939.6 & 480.2 & 57.0 & -364.4 & -426.6 & -532.4 & -610.9 & -596.4 \\
\hline Use of Fund credit and loans to the Fund (net) & 0.0 & 118.6 & -4.5 & -17.4 & -24.6 & -22.0 & -27.6 & -44.3 & 0.0 \\
\hline Change in arrears $3 /$ & 147.2 & -2.4 & 0.0 & -61.4 & 0.0 & 0.0 & 0.0 & 0.0 & 0.0 \\
\hline Rescheduling /debt swap & 107.2 & 0.0 & 0.0 & 6.1 & 0.0 & 0.0 & 0.0 & 0.0 & 0.0 \\
\hline Remaining gap & 0.0 & 0.0 & 0.0 & 201.1 & 0.0 & 0.0 & 0.0 & 0.0 & 0.0 \\
\hline \multicolumn{10}{|l|}{ Memorandum items: } \\
\hline Gross official reserves (end of period) & $2,415.3$ & $3,354.9$ & $2,874.7$ & $2,817.7$ & $3,182.1$ & $3,608.7$ & $4,141.1$ & $4,752.0$ & $5,348.4$ \\
\hline In months of following year's imports & 3.0 & 3.3 & 3.3 & 2.9 & 2.9 & 3.0 & 3.1 & 3.2 & 3.3 \\
\hline Change in reserves due to valuation ("-" represents loss) 4 / & 6.1 & 40.4 & -337.3 & $\ldots$ & $\ldots$ & $\ldots$ & $\ldots$ & $\ldots$ & ... \\
\hline \multicolumn{10}{|l|}{ Current account balance } \\
\hline (Percent of GDP, excluding official transfers) & -2.8 & -4.3 & -6.7 & -3.6 & -4.8 & -4.6 & -4.6 & -3.9 & -3.6 \\
\hline (Percent of GDP, including official transfers) & -2.5 & -4.1 & -6.7 & -3.7 & -4.8 & -4.7 & -4.6 & -3.9 & -3.6 \\
\hline Import volume growth, goods (percent) & 10.5 & 10.2 & 4.9 & 6.8 & 6.8 & 8.8 & 8.1 & 8.0 & 9.1 \\
\hline Export volume growth, goods (percent) & -4.1 & 11.9 & 9.5 & 7.2 & 6.9 & 11.1 & 9.9 & 11.3 & 11.4 \\
\hline
\end{tabular}

Sources: Kenyan authorities; and staff estimates and projections.

1/ The foreign travel credit is comprised of two components, recorded tourism inflows and an estimate of additional under-reported tourism receipts.

2/ Historical figures include errors and omissions. government's budget presentation does not show changes to arrears associated with disputed security-related contracts.

4/ Preliminary figures. 
Table 5. Kenya: External Financing Requirements and Resources, 2006/07-2009/10 ${ }^{1 /}$

(In millions of U. S. dollars)

\begin{tabular}{lrrrr}
\hline & $2006 / 07$ & $2007 / 08$ & $2008 / 09$ & $2009 / 10$ \\
\hline External financing requirements & -1401.0 & -2480.6 & -1373.3 & -1994.7 \\
Current account (excl.official grants) & -849.3 & -1465.2 & -1769.8 & -1458.3 \\
Scheduled amortization (official) & -251.4 & -265.1 & -241.9 & -254.2 \\
IMF payments, gross & 0.0 & -4.5 & -8.7 & -17.3 \\
Change in arrears, net & 69.9 & -26.0 & 0.0 & -61.4 \\
Change in gross official reserves (- is buildup) & -370.2 & -719.8 & 647.1 & -203.5 \\
Resources & 1401.0 & 2480.6 & 1222.5 & 1944.4 \\
Program support (committed and tentatively identified) & 59.3 & 134.1 & 0.0 & 0.0 \\
IMF & 59.3 & 59.3 & 0.0 & 0.0 \\
Program loans & 0.0 & 20.0 & 0.0 & 0.0 \\
$\quad$ African Development Bank (ADB) & 0.0 & 0.0 & 0.0 & 0.0 \\
IDA & 0.0 & 20.0 & 0.0 & 0.0 \\
$\quad$ Other & 0.0 & 0.0 & 0.0 & 0.0 \\
Program grants & 0.0 & 54.8 & 0.0 & 0.0 \\
Project Support & 377.2 & 650.9 & 834.9 & 1103.2 \\
Project loans & 153.3 & 325.0 & 515.1 & 662.5 \\
Project grants & 223.9 & 325.9 & 319.7 & 440.7 \\
Government-guaranteed, commercial loans & 93.1 & 83.5 & 58.1 & 56.3 \\
Private financing, net & 160.7 & 543.2 & 825.9 & 785.0 \\
$\quad$ of which, foreign direct investment & 622.7 & 891.7 & 658.1 & 466.5 \\
Errors and omissions & 657.1 & 1069.0 & -502.4 & 0.0 \\
Rescheduling / debt swap & 53.6 & 0.0 & 6.1 & 0.0 \\
Remaining gap & 0.0 & 0.0 & -151 & -50 \\
\hline Sources: Kenyan authorities and staff & & & &
\end{tabular}

Sources: Kenyan authorities and staff estimates and projections.

$1 /$ The fiscal year is July/June. 
Table 6. Financial Soundness Indicators, 2003-08

\begin{tabular}{|c|c|c|c|c|c|c|c|c|}
\hline & & & & & & & 2008 & \\
\hline & Dec-03 & Dec-04 & Dec-05 & Dec-06 & Dec-07 & Mar-08 & May-08 & Dec-08 \\
\hline \multicolumn{9}{|l|}{ Capital } \\
\hline Regulatory capital to risk weighted assets & 17.3 & 16.6 & 16.4 & 16.5 & 18.0 & 19.5 & 17.4 & 18.9 \\
\hline Regulatory Tier 1 capital to risk weighted assets & 16.3 & 16.2 & 16.0 & 16.4 & 16.8 & 18.3 & 16.2 & 16.9 \\
\hline \multicolumn{9}{|l|}{ Asset Composition and Quality } \\
\hline Non-performing loans to gross loans ${ }^{1}$ & 34.9 & 29.3 & 25.6 & 21.3 & 10.9 & 10.5 & 9.6 & 8.4 \\
\hline Non-performing loans net of provisions to total capital & 60.7 & 52.7 & 40.1 & 28.6 & 15.1 & 15.5 & 15.4 & 11.3 \\
\hline \multicolumn{9}{|l|}{ Earnings and Profitability } \\
\hline Return on assets (annualized) & 2.3 & 2.1 & 2.4 & 2.8 & 3.0 & 3.6 & 3.2 & 2.8 \\
\hline Return on equity (annualized) & 23.2 & 22.0 & 25.0 & 28.6 & 27.5 & 33.3 & 33.7 & 25.2 \\
\hline \multicolumn{9}{|l|}{ Liquidity } \\
\hline Liquid assets to total assets & 33.2 & 32.4 & 33.1 & 30.5 & 35.1 & 36.1 & 38.6 & 34.4 \\
\hline Liquid assets to total short-term liabilities & 48.9 & 41.5 & 40.6 & 44.4 & 40.2 & 40.2 & 41.0 & 37.0 \\
\hline \multicolumn{9}{|l|}{ Sensitivity Analysis } \\
\hline Net open positions in FX to capital & 12.0 & 8.0 & 6.0 & 7.1 & 5.4 & 7.0 & 7.3 & 5.6 \\
\hline
\end{tabular}

Source: Central Bank of Kenya

${ }^{1}$ The ratios were computed using gross non-performing loans and gross loans. 
Table 7. Kenya Indicators of Capacity to Repay the Fund, 2008-19

\begin{tabular}{|c|c|c|c|c|c|c|c|c|c|c|c|c|}
\hline & \multirow{2}{*}{2008} & \multicolumn{11}{|c|}{ Projections } \\
\hline & & $2009^{3}$ & 2010 & 2011 & 2012 & 2013 & 2014 & 2015 & 2016 & 2017 & 2018 & 2019 \\
\hline \multicolumn{13}{|l|}{$\begin{array}{l}\text { Fund obligations based on existing credit } \\
\text { (in millions of SDRs) }\end{array}$} \\
\hline Principal & 6.72 & 10.36 & 16.72 & 15.00 & 18.75 & 30.00 & 25.00 & 20.00 & 15.00 & 11.25 & 0.00 & 0.00 \\
\hline Charges and interest & 0.84 & 0.92 & 0.88 & 0.79 & 0.71 & 0.59 & 0.45 & 0.33 & 0.26 & 0.19 & 0.16 & 0.16 \\
\hline \multicolumn{13}{|c|}{$\begin{array}{l}\text { Fund obligations based on existing and prospective credit } \\
\text { (in millions of SDRs) }\end{array}$} \\
\hline Principal & 6.72 & 10.36 & 16.72 & 15.00 & 18.75 & 30.00 & 38.57 & 47.14 & 42.14 & 38.39 & 27.14 & 13.57 \\
\hline Charges and interest & 0.84 & 1.37 & 1.56 & 1.47 & 1.39 & 1.27 & 1.11 & 0.89 & 0.68 & 0.47 & 0.30 & 0.18 \\
\hline \multicolumn{13}{|c|}{ Total obligations based on existing and prospective credit ${ }^{1}$} \\
\hline In millions of SDRs & 7.56 & 11.73 & 18.28 & 16.47 & 20.14 & 31.27 & 39.68 & 48.03 & 42.82 & 38.86 & 27.44 & 13.75 \\
\hline In millions of US dollars & 11.95 & 17.99 & 28.12 & 25.43 & 31.20 & 48.61 & 61.89 & 74.91 & 66.79 & 60.61 & 42.80 & 21.45 \\
\hline In percent of exports of goods and services ${ }^{2}$ & 0.15 & 0.25 & 0.35 & 0.28 & 0.31 & 0.42 & 0.47 & 0.51 & 0.40 & 0.32 & 0.20 & 0.09 \\
\hline In percent of debt service & 3.33 & 4.79 & 6.68 & 5.39 & 6.05 & 7.83 & 8.97 & 8.81 & 6.63 & 5.17 & 3.29 & 1.50 \\
\hline In percent of quota & 2.79 & 4.32 & 6.74 & 6.07 & 7.42 & 11.52 & 14.62 & 17.70 & 15.78 & 14.32 & 10.11 & 5.07 \\
\hline In percent of gross international reserves & 0.42 & 0.64 & 0.88 & 0.70 & 0.75 & 1.02 & 1.16 & 1.24 & 0.99 & 0.79 & 0.50 & 0.22 \\
\hline \multicolumn{13}{|l|}{ Outstanding Fund credit ${ }^{1}$} \\
\hline In millions of SDRs & 162.08 & 287.42 & 270.70 & 255.70 & 236.95 & 206.95 & 168.38 & 121.24 & 79.10 & 40.71 & 13.57 & 0.00 \\
\hline In millions of US dollars & 256.28 & 440.69 & 416.47 & 394.75 & 367.11 & 321.70 & 262.62 & 189.10 & 123.37 & 63.49 & 21.16 & 0.00 \\
\hline In percent of exports of goods and services & 3.11 & 6.01 & 5.24 & 4.40 & 3.67 & 2.80 & 2.01 & 1.28 & 0.74 & 0.34 & 0.10 & 0.00 \\
\hline In percent of debt service & 71.42 & 117.42 & 98.94 & 83.65 & 71.21 & 51.82 & 38.05 & 22.25 & 12.24 & 5.42 & 1.63 & 0.00 \\
\hline In percent of quota & 59.72 & 105.90 & 99.74 & 94.22 & 87.31 & 76.25 & 62.04 & 44.67 & 29.15 & 15.00 & 5.00 & 0.00 \\
\hline In percent of gross international reserves & 8.91 & 15.64 & 13.09 & 10.94 & 8.87 & 6.77 & 4.91 & 3.14 & 1.82 & 0.83 & 0.25 & 0.00 \\
\hline \multicolumn{13}{|l|}{ Memorandum items: } \\
\hline Exports of goods and services (millions of US dollars) & 8,228 & 7,331 & 7,953 & 8,971 & 10,012 & 11,498 & 13,039 & 14,719 & 16,622 & 18,776 & 21,154 & 23,840 \\
\hline Debt service (millions of US dollars) & 359 & 375 & 421 & 472 & 516 & 621 & 690 & 850 & 1,008 & 1,173 & 1,301 & 1,429 \\
\hline Quota (millions of SDRs) & 271 & 271 & 271 & 271 & 271 & 271 & 271 & 271 & 271 & 271 & 271 & 271 \\
\hline Gross international reserves (millions of US dollars) & 2,875 & 2,818 & 3,182 & 3,609 & 4,141 & 4,752 & 5,348 & 6,020 & 6,775 & 7,625 & 8,583 & 9,660 \\
\hline GDP (millions of US dollars) & 30,229 & 29,764 & 36,280 & 42,621 & 47,443 & 52,081 & 57,587 & 62,482 & 67,793 & 73,555 & 79,807 & 86,591 \\
\hline
\end{tabular}

Source: Fund staff estimates and projections.

1. Includes possible ESF disbursement of 135.7 million SDR, 50\% of quota, in 2009

2. Total debt service includes IMF repurchased and repayments.

3. April 1 - December 31, 2009 


\section{Appendix. Letter of Intent}

Nairobi, May 15, 2009

Mr. Dominique Strauss-Kahn

Managing Director

International Monetary Fund

Washington, D.C. 20431

U.S.A.

Dear Mr. Strauss-Kahn:

In response to bold economic and structural reforms implemented by the Government over the Economic Recovery Strategy (ERS) period, 2003-07, the Kenyan economy staged a remarkable recovery with real GDP growth reaching 7 percent in 2007, up from 0.5 percent in 2002. This growth rate, which compared well with those achieved by most reforming countries in sub-Saharan Africa, had a positive impact on the welfare of Kenyans, with per capita income rising from about US\$ 350 in 2002 to over US\$ 700 by 2007.

However, in 2008, the economy suffered major shocks that threatened to reverse the gains we had achieved over the ERS period. Post-election violence in early 2008 impacted negatively on key sectors of our economy such as tourism, manufacturing, transport and agriculture, resulting in a year-on-year decline in real GDP of 1 percent in the first quarter of 2008. While the economy showed signs of quick recovery during the subsequent quarter, these hopes were diminished as the country was buffeted by record high fuel and fertilizer prices. These shocks were compounded by the failure of the short rains in October-November 2008, resulting in a sharp decline in domestic food supplies, particularly, maize - a key staple food for our population. Consequently, an estimated 10 million Kenyans have become food insecure and millions are at risk of starvation. The government and the private sector need to import about one million tons of maize and other cereals between February and August 2009. At the same time, the economy began to feel the adverse effects of the global financial crisis which has slowed recovery of our tourism sector and reduced remittances and private capital inflows.

\section{Impact of the shocks}

These shocks have put severe pressure on our fiscal position, the balance of payments and the exchange rate. The international reserves of the Central Bank of Kenya (CBK) have fallen to below 3 months of import cover from about 4 months a year ago and are expected to remain under pressure in 2009. 
The global financial crisis has made it difficult to issue a planned sovereign bond in the international capital market to finance our development expenditures. This, together with diminished prospects for privatization receipts and lower revenues arising from a weaker economy, has given rise to a substantial financing shortfall for the 2008/9 budget.

Recession in advanced countries is likely to restrain, if not reduce, demand for Kenya's exports, including tea, horticulture and coffee in 2009 and, if protracted, in the medium-term. Moreover, tourism earnings, remittances and private capital inflows are expected to decline. These developments will, notwithstanding the decline in oil and fertilizer prices, put further pressure on our external balance of payments, exchange rate and official foreign exchange reserves in 2008/9.

These shocks are threatening to derail our economic recovery and pose substantial challenges for our macroeconomic policy management. Inflationary pressure associated with food shortages remains high and is not expected to dissipate at least until the long rains are received to boost food supplies after August. Thus, increased importation of maize will further erode our official foreign exchange reserves and put pressure on the exchange rate at a time when our prospects for exports earnings, remittances, and capital inflows are being threatened by the negative impact of the global financial crisis

While we are yet to feel the full impact of the global financial crisis - especially its second round effects on the real economy, Kenya is likely to face a more difficult time in 2009/10 with low economic growth, increased unemployment and continued high prices for maize if rains become inadequate. We are thus faced with the task of finding solutions to sustain the economy and protect the livelihood of the poor.

\section{Policies to address the shocks}

Given our commitment to maintaining macro-economic stability, reigning in inflation and sustaining growth crucial for poverty reduction, we had to take immediate action to mitigate the impact of the shocks arising from drought, higher world prices of food, fuel and fertilizer, and the global financial crisis. We have taken concerted fiscal, monetary and administrative/structural measures and will be implementing additional measures in the coming year:

- The Government has withdrawn the generalized maize subsidy scheme introduced in November 2008 in response to rising maize prices and partially liberalized the maize market in Kenya. Because of design flaws, the scheme led to diversion of maize from the National Cereal and Produce Board (NCPB) to unintended purposes. Going forward, a new well-targeted food subsidy program that avoids price distortions and minimizes opportunities for abuse will be developed by December 2009. As the new scheme will not need the support of an export ban, we intend to eliminate the ban on maize exports imposed in October 2008 by end-December 2009. 
- Effective February 2009, the government removed the duty and VAT on maize imports for a period of six months to allow the private sector to import maize at lower cost to bridge the food deficit. The Government will reassess the food situation with a view of granting an extension of duty-free importation of maize, if the situation dictates so.

- On the fiscal front, the government is taking action to protect critical expenditures and boost economic activity while ensuring medium-term debt sustainability. Because of the revenue shortfall, the postponement of the planned sovereign bond issuance, and additional expenditures for drought relief, a financing gap for the financial year ending June 2009 has emerged. The government will close the gap by rationalizing expenditures, cutting non-priority expenditures and increasing domestic borrowing. The increased borrowing is necessary to maintain expenditures on key infrastructure projects and poverty reduction, as well as to support economic activity in the face of the adverse impact of the global financial crisis. Despite the higher domestic financing, the overall fiscal deficit (including grants) for 2008/09 is about 5 percent of GDP based on the current implementation rate for foreign financed projects - smaller than budgeted but moderately larger than in 2007/08. We recognize the downside risk of a larger-than-projected revenue shortfall. Should this risk materialize, we would take further actions such as reprioritizing expenditures to keep within the current target for net domestic borrowing. For 2009/10, we will continue to stimulate the economy. To this end, we plan to borrow from domestic sources about Ksh 109 billion (4.2 percent of GDP), which we believe can be accommodated by the domestic financial market. Despite these temporary increases in domestic borrowing, we remain committed to reducing the debt-to-GDP ratio in the medium term.

- In the monetary area, to ease the liquidity situation, the CBK has cut its policy ratethe Central Bank Rate (CBR) - by a total of 75 percentage point since December and lowered banks' reserve requirement ratio from 6 percent to 5 percent. Going forward, the revised monetary program for 2008/9 is expected to reign in inflation, while providing sufficient liquidity to support economic activity and allowing for a gradual rebuilding of international reserves.

- In view of the challenges in gauging monetary policy under the current methodology of measuring the consumer price index, the Kenya National Bureau of Statistics began in February 2009 to undertake a Point of Sale Survey (PSS) based on an updated consumer basket. These price data are needed to determine the price reference period for the new CPI, and to finalize CPI rebasing and revision exercise. We expect to review and audit the implementation of the new series; and thereafter to launch a new CPI with a revised compilation methodology by end-May 2009.

- To address the increased risks to the financial sector, the CBK is intensifying its oversight to ensure that banks are making adequate and timely provision for bad and doubtful loans, and taking an increasingly proactive approach to surveillance and enforcement. The government will bring the Banking Bill before Parliament before the end of the next Parliamentary session — enacting this bill would strengthen the 
hand of the CBK by authorizing consolidated supervision and prompt corrective action, which will be crucial if asset quality deteriorates.

- Regarding the external sector, as the balance of payments starts improving in $2009 / 10$, the central bank will pursue a strategy of gradually rebuilding the official foreign exchange reserves consistent with the reserve money path that will reduce inflation to 5 percent by June 2010. We will maintain a managed-float exchange rate regime, which has served Kenya well in terms responding to external shocks. The CBK will continue to limit its interventions in the foreign exchange market only to smoothing excessive short-term volatilities. In view of the importance of closely monitoring the transactions in the balance of payments as the impact of the global financial crisis unfolds, the KNBS is committed to undertaking a foreign capital flow survey by October 2009 to track foreign direct investment and other capital flows. Already preparatory work including identification of sampling frame and trainings has been done.

- We are committed to zero tolerance of corruption. In this regard, the Government will continue to advance governance reforms within the context of updated Governance Action Plan (GAP). Recent corruption allegations related to the sale of maize from the National Cereal and Produce Board (NCBP) to middlemen are being investigated by the Kenya Anti-Corruption Commission (KACC). In the meantime, the Government has instituted changes in the management of NCPB and launched forensic audit of NCPB operations that will inform further actions. With regard to mismanagement of the distribution of fuel associated with the State-owned Kenya Pipeline Corporation (KPC) and private petroleum dealers, the Government has taken swift corrective actions. It replaced the management at KPC and changed the procedures for allocation of storage space to oil suppliers at the Kipevu storage facility, and the KACC has launched an investigation.

- We will continue to pursue structural reforms to improve our long-term growth potential. Our priority will be given to the enactment of the Public Financial Management Bill and establishing its enabling regulations; improving treasury cash management (including making operational the treasury single account); strengthening debt management; improving fiscal reporting (including increasing the functionality of IFMIS); and furthering procurement reforms.

The Government stands ready to implement other policy measures should the shocks turn out to be worse than expected.

\section{Request for Fund assistance under ESF}

The Government has declared the food situation a national disaster. The WFP has completed a comprehensive assessment of the food situation and the interventions required. So far, we have received support from China of US\$3 million and we expect other development partners, including the World Bank and the AfDB, to channel their support through the WFP mechanism to help mitigate the food problem. The NCPB is expected to 
import 3 million bags of maize under a commodity credit guarantee scheme provided by the United States Department of Agriculture. A number of other donors are providing support through existing portfolio with the aim of improving domestic agricultural productivity in a sustainable manner.

Despite these anticipated increases in donor support, the challenges outlined earlier require additional urgent assistance, particularly with regard to bolstering our international reserves. We therefore request that the IMF allow the Kenyan government the maximum access of 50 percent of quota through the Rapid-Access Component of the Exogenous Shocks Facility (ESF) to address these challenges. Further, we will continue to engage with the Fund on policy advice and have no intention of introducing new exchange and trade restriction. Similarly, we will maintain full pass-through of world prices for petroleum products. We expect to update the Financial Sector Assessment Program (FSAP) following our earlier request. We also request an update of the safeguard assessment for the Central Bank of Kenya.

The Government of Kenya authorizes the IMF to make this letter and the IMF staff report available to the public, including through the IMF internet website.

Sincerely yours,

$/ \mathrm{s} /$

Uhuru M. Kenyatta

Deputy Prime Minister and Minister for

Finance /s/

Njuguna Ndung'u Governor

Central Bank of Kenya 
INTERNATIONAL MONETARY FUND

KENYA

Staff Report for Request for Disbursement Under the Rapid-Access Component of the Exogenous Shocks Facility

Informational Annex

Prepared by the African Department

(In consultation with other departments)

Approved by Saul Lizondo and Dominique Desruelle

May 15, 2009

Contents

Page

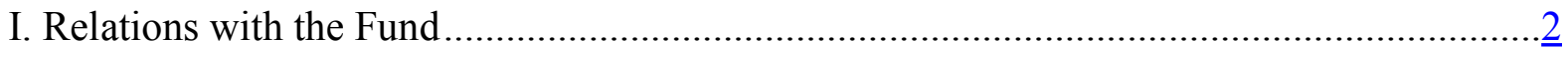

II. Joint Management Action Plan 2008-09 .................................................................

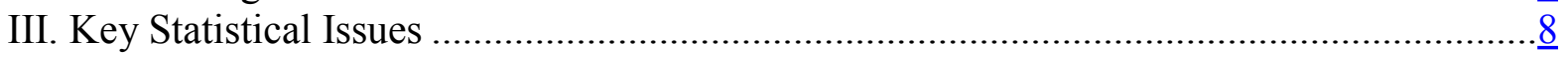




\section{Kenya: Relations with the Fund \\ (As of March 31, 2009)}

I. Membership Status: Joined February 3, 1964; Article VIII.

II. General Resources Account:

Quota

Fund holdings of currency

Reserve position in Fund

III. SDR Department:

Net cumulative allocation

Holdings

IV. Outstanding Purchases and Loans:

Poverty Reduction and Growth Facility (PRGF) arrangements
SDR million

271.40

258.55

12.86

SDR million

36.99

0.53

162.08
1.44

Percent of quota

\section{Percent of quota}

100.00

95.26

4.74

Percent of allocation

100.00

59.72

V. Latest Financial Arrangements:

Approval Expiration Amount Approved Amount Drawn

Type

$\underline{\text { Date }}$

$\underline{\text { Date }}$

$\underline{(\mathrm{SDR} \text { million})}$

$\underline{(\text { SDR million) }}$

PRGF

$11 / 21 / 03$

$11 / 20 / 07$

150.00

150.00

PRGF

$8 / 4 / 00$

$8 / 3 / 03$

190.00

149.55

33.60

24.93

ESAF

$2 / 22 / 93$

$4 / 25 / 99$

45.23

ESAF

$5 / 15 / 89$

261.40

$3 / 31 / 93$

45.23

216.17 
The last three-year PRGF arrangement was approved on November 21, 2003, in the amount of SDR 175 million. Access was augmented to SDR 225 million at the time of the first review on December 20, 2004, to address the impact of a drought and higher oil prices. In light of a balance of payments improvement and at the request of the authorities, access was reduced to SDR 150 million at the time of the second review. The arrangement expired on November 20, 2007.

\section{Projected Obligations to Fund}

(SDR million; based on existing use of resources and present holdings of SDRs):

\begin{tabular}{lrrrrr} 
& 2009 & 2010 & 2011 & 2012 & 2013 \\
Principal & 10.36 & 16.72 & 15.00 & 18.75 & 30.00 \\
Charges/interest & 0.92 & 0.88 & 0.79 & 0.71 & 0.59 \\
Total & 11.28 & 17.60 & 15.79 & 19.46 & 30.59 \\
\hline
\end{tabular}

VII. Implementation of HIPC Initiative: Not Applicable

VIII. Implementation of Multilateral Debt Relief Initiative (MDRI): Not Applicable

\section{Safeguards Assessments}

An update safeguards assessment of the Central Bank of Kenya (CBK) is underway with respect to the authorities request for a disbursement under the Rapid-Access Component of the Exogenous Shocks Facility (ESF). The CBK was subject previously to a safeguards assessment with respect to the augmentation of access under the PRGF Arrangement approved on December 20, 2004. The assessment, completed on September 8, 2005, noted that the CBK has taken steps to implement the recommendations of the earlier safeguards assessments and proposed measures to address the remaining vulnerabilities.

\section{Exchange Arrangements}

"Kenya 's currency is the shilling, which floats against other currencies. The official exchange rate, which is set at the previous day's average market rate, applies only to government and government-guaranteed external debt-service payments and to government imports for which there is a specific budget allocation. The exchange rate regime is a managed float, in which the U.S. dollar is the principal intervention currency. Kenya has accepted the obligations of Article VIII, Sections 2, 3, and 4, and maintains an exchange system free of restrictions on payments and transfers for current international transactions, other than restrictions notified to the Fund under Decision No. 144 (52/51). On May 15, 2009, the exchange rate was K Sh $78.3=\mathrm{US} \$ 1.00$. 


\section{Article IV Consultations}

Upon expiration of the PRGF program in November 2007, Kenya has now reverted back to a standard 12-month cycle for Article IV consultations. The last Article IV consultation was concluded on September 10, 2008 (Country Report No. 08/339).

\section{FSAP Participation}

The joint IMF-World Bank Financial Sector Assessment Program (FSAP) missions took place in Nairobi in July and October, 2003. The staff report on the Financial Sector Stability Assessment (FSSA) was issued on November 29, 2004. An update of the FSAP is planned for October 2009.

XIII. Technical Assistance

\begin{tabular}{|c|c|c|}
\hline Department & Purpose & Time of Delivery \\
\hline MFD & Central Bank Accounting & February 2004 \\
\hline STA & Government Finance Statistics (GDDS) & February 2004 \\
\hline FAD & Customs Administration & March 2004 \\
\hline FAD & Tax Administration & March/April 2004 \\
\hline FAD & $2^{\text {nd }}$ World Bank/AFRITAC East PEM & \\
\hline & Assessment and Action Plan (AAP) Mission & May 2004 \\
\hline FAD & $\begin{array}{l}\text { AFRITAC East Treasury Reforms mission } \\
\text { (implementation follow up in July 2004, } \\
\text { February-March 2005, September 2005, } \\
\text { September 2006) }\end{array}$ & June 2004 \\
\hline MFD & $\begin{array}{l}\text { Banking Supervision/Deposit Insurance } \\
\text { /Legislation }\end{array}$ & July 2004 \\
\hline FAD & AFRITAC East Aid Flow Tracking Mission & July 2004 \\
\hline FAD & Revenue Administration & September/October 2004 \\
\hline MFD & Deposit Insurance/Legislation (with LEG) & October 2004 \\
\hline MFD & $\begin{array}{l}\text { Public Debt Management and Monetary } \\
\text { Policy Implementation }\end{array}$ & November 2004 \\
\hline STA & ROSC Mission & January 2005 \\
\hline MFD & $\begin{array}{l}\text { Monetary Framework, Monetary Operations, } \\
\text { and Banking Supervision }\end{array}$ & October 2005 \\
\hline FAD & Revenue Administration & November 2005 \\
\hline FAD & $\begin{array}{l}\text { AFRITAC East Semi-Autonomous Agencies } \\
\text { and EBFs/Fiscal Reporting Mission }\end{array}$ & March 2006 \\
\hline FAD & $\begin{array}{l}\text { HQ Mission: Strengthening the Budget and } \\
\text { Reporting System }\end{array}$ & August 2006 \\
\hline FAD & $\begin{array}{l}\text { AFRITAC East TA: Revision of Public } \\
\text { Finance Management Act }\end{array}$ & October/November 2006 \\
\hline $\mathrm{MCM}$ & $\begin{array}{l}\text { AFRITAC East TA: Review of Risk-Based } \\
\text { Supervision Manual }\end{array}$ & November/December 2006 \\
\hline $\mathrm{MCM}$ & Monetary Operations TA & January 2007 \\
\hline
\end{tabular}




$\begin{array}{lll}\text { STA } & \text { External Debt Statistics Workshop } & \text { February / March 2007 } \\ \text { FAD } & \text { AFRITAC East TA: Customs Administration } & \text { July 2007 } \\ \text { FAD } & \text { Fiscal ROSC } & \text { July 2007 } \\ \text { MCM } & \text { Payment Systems } & \text { February 2008 } \\ \text { STA } & \text { Consumer Price Statistics } & \text { March 2008 } \\ \text { MCM } & \text { Monetary Operations/Banking } & \text { April 2008 } \\ \text { STA } & \text { National Accounts } & \text { April 2008 } \\ \text { FAD } & \text { AFRITAC East TA: Budget } & \text { May 2008 } \\ \text { STA/MCM } & \text { Financial Soundness Indicators } & \text { June 2008 } \\ \text { FAD } & \text { Budgeting } & \text { July 2008 } \\ \text { MCM } & \text { Bond-Pricing Techniques Workshop } & \text { July 2008 } \\ \text { FAD } & \text { AFRITAC East TA: Regional PFM Advisor: } & \\ & \text { Budget and Tax review } & \text { August 2008 } \\ \text { FAD } & \text { AFRITAC East TA: Customs Administration } & \\ \text { MCM } & \text { Payment Systems } & \text { August 2008 } \\ \text { STA } & \text { AFRITAC East TA: Monetary and Financial } & \\ & \text { Statistics } & \text { August 2008 } \\ \text { FAD } & \text { Tax Administration Workshop } & \text { November 2008 } \\ \text { FAD } & \text { PFM and Fiscal Decentralization } & \text { November 2008 } \\ \text { STA } & \text { Reweighing and Rebasing of CPI } & \text { December 2008 } \\ \text { STA } & \text { Producer Price Index compilation } & \text { December 2008 } \\ \text { MCM } & \text { Risk based Supervision } & \text { January 2009 } \\ \text { MCM } & \text { Capital market Regulation and Supervision } & \text { March 2008 } \\ & & \\ & & \end{array}$

\section{Resident Representative}

The Fund has had a resident representative in Kenya since December 1993.

Mr. W. Scott Rogers is the current Senior Resident Representative. 
II. Kenya: IMF-World Bank Joint Management Action Plan 2009-10

\begin{tabular}{|c|c|c|c|}
\hline Title & Products /Activity & $\begin{array}{l}\text { Provisional timing } \\
\text { of mission (if } \\
\text { relevant) }\end{array}$ & $\begin{array}{l}\text { Expected } \\
\text { delivery date }\end{array}$ \\
\hline \multicolumn{4}{|c|}{ I. Mutual Information on Relevant Work Program } \\
\hline \multirow{10}{*}{$\begin{array}{l}\text { Bank Work } \\
\text { Program }\end{array}$} & A. Strategy and Analytical Work & & \\
\hline & Poverty and Inequality Assessment & July 2008 & June 2009 \\
\hline & Public Expenditure Notes & Jan-Feb 2009 & June 2009 \\
\hline & $\begin{array}{l}\text { Staff Advisory Note on the Medium-Term (2008- } \\
\text { 12) Strategy for National Transformation }\end{array}$ & No mission needed & \\
\hline & Agricultural Policy Review & To be decided & Completed \\
\hline & Land Reforms Study & & June 2009 \\
\hline & Parliament's Role in Governance & & Completed \\
\hline & Police Oversight Mechanisms & & Completed \\
\hline & B. Ongoing and New Projects & & \\
\hline & $\begin{array}{l}\text { Several projects on public sector reforms } \\
\text { including legal and judicial, capacity building, } \\
\text { agriculture, natural resource management, } \\
\text { transportation, energy, urban services, small } \\
\text { and medium scale enterprises, education and } \\
\text { health (including HIV/AIDS) }\end{array}$ & Several & Ongoing \\
\hline
\end{tabular}

IMF Work

Program

\section{A. Missions}

Article IV Consultation

Possible discussion of an IMF-supported program

Financial Sector Assessment Program (FSAP) Update

August 2009

October 2009

November 2009

January 2010

October 2009

December 2009

\section{B. Analytical Work}

SIP on Financial Markets

AFR-MCM Project on EAC Financial market integration

August 2009

October 2009

No mission needed

October 2009

February 2009

Completed

February 2009

Completed

February 2009

Completed

February 2009

Completed

March 2009

Completed

March 2009
Completed 


\begin{tabular}{|c|c|c|c|}
\hline Title & Products /Activity & $\begin{array}{l}\text { Provisional timing } \\
\text { of mission (if } \\
\text { relevant) }\end{array}$ & $\begin{array}{l}\text { Expected } \\
\text { delivery date }\end{array}$ \\
\hline & Public Asset/Liability Management (Public Debt) & March 2009 & May 2009 \\
\hline & CPI Statistics Mission & May 2009 & August 2009 \\
\hline & Bank Supervision & June 2009 & $\begin{array}{l}\text { September } \\
2009\end{array}$ \\
\hline & Capital Markets / Securities Supervision & July 2009 & $\begin{array}{l}\text { September } \\
2009\end{array}$ \\
\hline & Debt Management issues & September 2009 & $\begin{array}{l}\text { December } \\
2009\end{array}$ \\
\hline & Balance of Payments Statistics & September 2009 & $\begin{array}{l}\text { December } \\
2009\end{array}$ \\
\hline & National Accounts Statistics & October 2009 & January 2010 \\
\hline & Anti Money Laundering Diagnostic Mission & February 2010 & April 2010 \\
\hline & Balance of Payments Statistics & February 2010 & April 2010 \\
\hline \multicolumn{4}{|c|}{ II. Request for Work Program Inputs (as needed) } \\
\hline \multirow[t]{2}{*}{$\begin{array}{l}\text { Bank Request } \\
\text { to Fund }\end{array}$} & $\begin{array}{l}\text { Medium-term macro-economic and fiscal } \\
\text { framework to inform Public Expenditure Notes }\end{array}$ & $\begin{array}{l}\text { To share when } \\
\text { requested }\end{array}$ & Ongoing \\
\hline & $\begin{array}{l}\text { Profile of government's contingent liabilities in } \\
\text { non-financial public sector }\end{array}$ & $\begin{array}{l}\text { To share data when } \\
\text { available }\end{array}$ & $2008 / 09$ \\
\hline \multirow[t]{4}{*}{$\begin{array}{l}\text { Fund Request } \\
\text { to Bank }\end{array}$} & $\begin{array}{l}\text { Food and fuel price increases - impact on } \\
\text { poverty and growth, policy assessment and } \\
\text { response. }\end{array}$ & $\begin{array}{l}\text { To share work done } \\
\text { for the Region; No } \\
\text { Kenya-specific work } \\
\text { planned. }\end{array}$ & Ongoing \\
\hline & $\begin{array}{l}\text { Investment needs and their implications for } \\
\text { development spending; quality of spending }\end{array}$ & $\begin{array}{l}\text { To share work } \\
\text { being done in } \\
\text { response to specific } \\
\text { requests. }\end{array}$ & Ongoing \\
\hline & $\begin{array}{l}\text { MTEF for growth and equity and PFM issues } \\
\text { relating to key social sectors }\end{array}$ & $\begin{array}{l}\text { Some analytical } \\
\text { work already } \\
\text { shared. Other work } \\
\text { as described in } \\
\text { Bank Work Program } \\
\text { will be made } \\
\text { available once } \\
\text { completed. }\end{array}$ & Ongoing \\
\hline & Financial sector development issues & $\begin{array}{l}\text { Work in partnership } \\
\text { with other donors } \\
\text { such as DFID will } \\
\text { be shared. }\end{array}$ & Ongoing \\
\hline
\end{tabular}

\section{Agreement on Joint Products}

Joint products $\quad$ Public and External Debt Sustainability Analysis $\quad$ April $2009 \quad$ May 2009




\section{KENYA: KEY STATISTICAL ISSUES}

31. Data provision has some shortcomings, but is broadly adequate for surveillance and program monitoring. Weaknesses in macroeconomic statistics reflect organizational and skill shortcomings, and inadequate resources. In line with the new Statistics Law, the government established the autonomous Kenya National Bureau of Statistics (KNBS) in 2007 to replace the Central Bureau of Statistics (CBS).

32. The Report on the Observance of Standards and Codes - Data Module (IMF Country Report No. 05/388) published on October 31, 2005 stated that methodological soundness is uneven across datasets and significant improvement is required in government finance statistics and national accounts. Accuracy and reliability do not receive adequate attention in any of the datasets, while accessibility of the disseminated macroeconomic statistics are adequate, except for the balance of payments statistics.

33. Kenya participates in the Fund's General Data Dissemination System (GDDS) and the GDDS project for Anglophone Africa. Metadata and detailed plans for improving the data over the short and medium term are posted on the Fund's Dissemination Standards Bulletin Board (DSBB). Kenya has received Fund technical assistance. Further assessment of capacity building requirements has been undertaken by the AFRITAC East Statistical Advisor.

34. Monetary, exchange rate, and some external data are published on a monthly and biannual basis by the KNBS in its Monthly Economic Review. Core financial data are also made available to the Fund on a regular basis. A detailed account of various sectoral activities and the corresponding statistical data are published annually by the KNBS in its Economic Survey.

\section{National accounts}

35. Data quality has deteriorated significantly because of budgetary and staff constraints at the KNBS. GDP is believed to be significantly underestimated, as important and increasing parts of the economy, such as the informal sector, nonagriculture subsistence, horticulture, and self-employed professionals are not properly covered. An STA peripatetic advisor assisted the authorities in rebasing the national accounts estimates at constant 2001 prices and compiling institutional accounts for the general government sector. As a result, national accounts estimates for the years 1996-2005 in current and constant (2001) prices) have been published. Quarterly national accounts are being developed with the assistance of the AFRITAC East. Quarterly GDP estimates are now published.

\section{Prices and production}

6. In early 2002, the KNBS (then CBS) began publication of a new national CPI (covering 13 urban towns), with 1997 as reference year and outdated weights and basket of items derived from the 1993-94 Household Budget Survey (HBS). Indices are produced for lower and middle/upper income groups in Nairobi and other cities. The index is compiled and published on a timely basis. No producer, export, or import price indices are produced. 
In March 2008, a TA mission assisted the authorities in identifying the methodological issues relating to the measurement of inflation. The current methodology of aggregating prices at the elementary level was found to impart a substantial upward bias on CPI measurement. In line with STA recommendations, the KNBS plans to switch to a formula consistent with international best practices in 2009, together with a rebasing and reweighing of the CPI basket.

\section{Government finance statistics (GFS)}

7. The data ROSC mission emphasized a number of key areas in GFS compilation that should be improved, including: (i) migration to the GFSM 2001 methodology;

(ii) broadening the coverage to include extrabudgetary and social security funds and report on a general government level; (iii) reconciliation of fiscal statistics from various sources to limit discrepancies; (iv) improve information on external financing, particularly on expenditure directly financed from abroad; (v) compilation and dissemination of monthly and quarterly budget execution data; and (vi) training of Ministry of Finance (MOF) staff in the GFS methodology.

8. Since the beginning of FY 2005/06, Kenya has followed a new economic classification of the budget based on GFSM 2001 (with assistance from the AFRITAC-East). However, serious delays have emerged in reporting, reflecting difficulties in establishing budget execution and accounting systems consistent with the new classification. To ensure timely reconciliation and monitoring of budget execution, the existing systems would need to be promptly upgraded and made operational. Progress has been made toward moving to the IFMIS. There are still important gaps in ensuring proper reconciliation of fiscal data from different sources, including from various units within the MOF. The discrepancies in budget outturn data (between deficit/surplus and financing) remain significant, and the recording of external financing and expenditure directly financed from abroad is still an important area for improvement. The government has taken some steps to initiate a project to rationalize/eliminate extrabudgetary funds, but the progress in compiling consolidated fiscal statistics has remained limited.

9. The country reports data to STA for the budgetary central government for inclusion in the GFS Yearbook, albeit with a significant lag - the last data reported were for the year ending June 2005. The KNBS compiles the aggregate annual GFS revenue and expenditure data for the budgetary central government based on detailed data in the reports of the Controller and Auditor General. The data submitted for publication in the 2007 GFS Yearbook was reported in GFSM 2001 format, using bridge tables developed by the technical assistance missions. The recent steps taken in the migration to GFSM 2001 may help reduce the significant differences between KNBS data and the data compiled by the MOF and reported to AFR for surveillance and program monitoring purposes. Monthly and quarterly data are regularly reported for inclusion in the IFS.

\section{Monetary statistics}

10. Progress has been shown in the implementation of the Monetary and Financial Statistics Manual (MFSM) and development of the standardized report forms (SRFs). The 
authorities submitted SRF test data; however, there has not been further progress. In March 2007, a STA mission established the standardized report form for the data on the central bank; reviewed and revised the reporting form and compilation notes for the other depository corporations (ODCs) in line with MFSM data requirements; and identified the coverage of the ODC subsector. The mission found misclassification of a number of central government deposits as private sector deposits. A major issue for future work relates to the expansion of the coverage of the ODCs to include the Savings and Credit Cooperatives (SACCOS). As of end-March, 2007, there were 3,800 SACCOs, accounting for about 30 percent of the total deposits of the banking system. When implemented, a new bill providing for increased supervision over SACCOs would generate data as part of the supervisory function. A followup mission in June 2007 conducted a workshop for officials of ODCs and established a system for reporting Form 1SR to the IMF. The new Standardized Reporting System for reporting ODCs' data to the central bank was also finalized.

\section{External sector statistics}

11. The KNBS compiles annual balance of payments statistics in Kenya shillings that are regularly reported to STA, although with considerable delay. In addition, the Central Bank of Kenya (CBK) compiles a complete set of annual balance of payments statistics in U.S. dollars, which are reported to AFR and used for programming and surveillance purposes. The two datasets are not entirely consistent, and Fund staff has strongly encouraged the authorities to reconcile them. More recently, the CBK also started to compile and publish quarterly balance of payments estimates.

12. Although the overall quality of trade data may be reasonably good, data for other current account and many financial account transactions are rather weak. Following the liberalization of the exchange system in 1993-94, gaps emerged in coverage. The compilation system (other than that used for compiling customs statistics), used since 1994, relies on reports from domestic banks and may result in a substantial under-recording of current earnings, including tourism receipts; investment flows of the private sector; as well as transactions that are settled via accounts held abroad.

13. Present estimates of direct and portfolio investment are believed to be substantially understated. The large positive errors and omissions in the central bank data that have emerged in the balance of payments since 1994 give rise to uncertainties as to the potential size of external obligations. The MOF compiles data covering public and publicly guaranteed external debt obligations to official and commercial creditors. This database does not take into account nonresident purchases of the government's domestic currency-denominated debt securities. In developing the loan-by-loan debt sustainability analysis (DSA) in 2002, Fund and World Bank staff identified several significant debt data problems that have been addressed by the authorities. Nevertheless, significant debt data management problems remain, along with more general issues in the area of external debt management and its integration in the budget formulation and expenditure management systems.

14. To help address these issues, in 2006 a technical assistance mission recommended introducing a foreign investment survey, enhancing the foreign exchange statistics survey, and using a common methodology, including for estimations, in the use of available data in the 
KNBS and CBK. In 2007, a DFID funded enterprise survey failed to materialize. There are plans to conduct a foreign investment survey in 2009, which planned DFID-funded and STA external sector missions will support.

15. Kenya does not report international investment position statistics to STA. 
KENYA. TABLE OF COMMON INDICATORS REQUIRED FOR SURVEILLANCE

(AS OF APRIL 14, 2009)

\begin{tabular}{|c|c|c|c|c|c|c|c|}
\hline & \multirow{2}{*}{$\begin{array}{c}\text { Date of } \\
\text { latest } \\
\text { observatio } \\
\mathrm{n}\end{array}$} & \multirow[t]{2}{*}{ Date received } & \multirow{2}{*}{$\begin{array}{l}\text { Frequency of } \\
\text { Data }^{6}\end{array}$} & \multirow{2}{*}{$\begin{array}{l}\text { Frequency of } \\
\text { Reporting }\end{array}$} & \multirow{2}{*}{$\begin{array}{l}\text { Frequency } \\
\text { of } \\
\text { publication }^{6}\end{array}$} & \multicolumn{2}{|c|}{ Memo Items $^{7}$} \\
\hline & & & & & & $\begin{array}{l}\text { Data Quality- } \\
\text { Methodological } \\
\text { soundness }\end{array}$ & $\begin{array}{l}\text { Data Quality-Accuracy } \\
\text { and reliability }\end{array}$ \\
\hline Exchange Rates & $4 / 14 / 09$ & $4 / 14 / 09$ & $\mathrm{D}$ & $\mathrm{D}$ & M & & \\
\hline $\begin{array}{l}\text { International Reserve Assets and Reserve } \\
\text { Liabilities of the Monetary Authorities }{ }^{1}\end{array}$ & $4 / 14 / 09$ & $4 / 14 / 09$ & $\mathrm{D}$ & $\mathrm{D}$ & M & & \\
\hline Reserve/Base Money & $4 / 14 / 09$ & $4 / 14 / 09$ & $\mathrm{D}$ & $\mathrm{D}$ & M & \multirow{4}{*}{ LO, LO, LO, LO } & \multirow{4}{*}{ LO, LO, O, O, NO } \\
\hline Broad Money & $2 / 28 / 09$ & $3 / 15 / 09$ & $M$ & $M$ & $M$ & & \\
\hline Central Bank Balance Sheet & $4 / 14 / 09$ & $4 / 14 / 09$ & D & $\mathrm{D}$ & M & & \\
\hline $\begin{array}{l}\text { Consolidated Balance Sheet of the Banking } \\
\text { System }\end{array}$ & 2/28/09 & $3 / 15 / 09$ & M & M & M & & \\
\hline Interest Rates ${ }^{2}$ & $3 / 30 / 09$ & $4 / 15 / 09$ & $\mathrm{D}$ & $\mathrm{D}$ & $M$ & & \\
\hline Consumer Price Index & 2/28/09 & $3 / 15 / 09$ & M & M & M & & \\
\hline $\begin{array}{l}\text { Revenue, Expenditure, Balance and } \\
\text { Composition of Financing }{ }^{3} \text {-General } \\
\text { Government }^{4}\end{array}$ & $3 / 2009$ & $4 / 2009$ & NA & NA & NA & LNO, LNO, LNO, LO & LNO, LO, LO, LO, NO \\
\hline $\begin{array}{l}\text { Revenue, Expenditure, Balance and } \\
\text { Composition of. Financing }{ }^{3-} \text { Central Government }\end{array}$ & $3 / 2009$ & $4 / 2009$ & Q & I & Q & & \\
\hline $\begin{array}{l}\text { Stocks of Central Government and Central } \\
\text { Government Guaranteed Debt }\end{array}$ & $3 / 30 / 08$ & $4 / 2009$ & Q & Q & Q & & \\
\hline External Current Account Balance & $12 / 2008$ & $2 / 2009$ & M & A & A & O, LO, O, LO & LNO ,LO, LO, LO, LO \\
\hline Exports and Imports of Goods and Services & $12 / 2008$ & $2 / 2009$ & M & Q & A & & \\
\hline GDP/GNP & 2008 Q3 & $2 / 2009$ & M & A & A & O, LNO, LNO, LO & LNO, LO, LNO, LO, LNO \\
\hline Gross External Debt & $3 / 30 / 08$ & $4 / 2009$ & M & Q & Q & & \\
\hline
\end{tabular}

${ }^{1}$ Includes reserve assets pledged or otherwise encumbered as well as net derivative positions

${ }^{2}$ Both market-based and officially determined, including discount rates, money market rates, rates on treasury bills, notes and bonds.

${ }^{3}$ Foreign, domestic bank, and domestic nonbank financing.

${ }^{4}$ The general government consists of the central government (budgetary funds, extra budgetary funds, and social security funds) and state and local governments.

${ }^{5}$ Including currency and maturity composition.

Daily (D), Weekly (W), Monthly (M), Quarterly (Q), Annually (A), Irregular (I); Not Available (NA).

${ }^{8}$ Reflects the should only be included for countries for which a Data ROSC (or a Substantive Update) has been prepared.

(the variable in each row. The assessment indicates whether international standards

列 definitions, scope, classification/ sectorization, and basis for recording are fully observed (O), largely observed (LO), largely not observed (LNO), or not observed (NO).

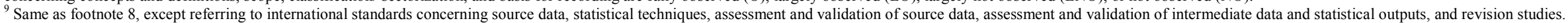




\title{
INTERNATIONAL DEVELOPMENT ASSOCIATION
}

\author{
INTERNATIONAL MONETARY FUND
}

\begin{abstract}
KENYA
Joint IMF/World Bank Debt Sustainability Analysis

Prepared by the Staffs of the International Monetary Fund and the World Bank

Approved by Saul Lizondo and Dominique Desruelle (IMF) and Carlos Braga and John Panzer (World Bank)
\end{abstract}

May 15, 2009

The debt sustainability analysis (DSA) was prepared jointly by Bank and Fund staffs in accordance with the standardized methodology for low-income countries. It is based on the general framework approved by the Executive Boards of the Fund and the World Bank in April 2005 and subsequently modified. ${ }^{3}$ The DSA has also benefited from consultation with African Development Bank staff. As in the previous DSA (Country Report No. 08/339), the findings indicate that the risk of external debt distress is low. Risks are somewhat greater for public debt, particularly in the event of lower growth.

\section{A. Methodology}

1. External debt sustainability is assessed in relation to policy-dependent debt burden thresholds. Kenya is classified as a medium performer in terms of the quality of its policies and institutions as measured by a three-year average of Kenya's score on the World Bank's Country Policy and Institutional Assessment (CPIA) index. ${ }^{4,5}$

2. The DSA is based on updated data provided by the authorities, available data on private sector debt, and estimates by the staffs. The DSA uses non-reconciled debt data and a single discount rate.

\footnotetext{
${ }^{3}$ http://www.imf.org/external/pubs/ft/dsa/lic.aspx

${ }^{4}$ http://go.worldbank.org/AXO6I14PK0

${ }^{5}$ For a medium performer, the indicative thresholds for external debt sustainability are an NPV of debt-to-GDP ratio of 40 percent, an NPV of debt-to-exports ratio of 150 percent, an NPV of debt-to-revenue ratio of 250 percent, a debt service-to-exports ratio of 20 percent, and a debt service-to-revenue ratio of 30 percent.
} 
3. This DSA consists of two parts - external and fiscal. The external DSA covers external debt of the central government (including parastatal borrowing with a government guarantee) and the central bank, and also includes estimates of private sector debt based on available information. The fiscal DSA covers total debt - external and domestic - incurred or guaranteed by the central government. ${ }^{6}$

\section{Key assumptions underlying the DSA are consistent with the Staff Report for Request for Access to the Rapid-Access Component of the Exogenous Shock Facility:}

- $\quad$ Average annual real GDP growth of 4.7 percent for 2008 through 2014, which is well below the 7.0 percent growth recorded in 2007 . Owing to the early 2008 political turmoil and the global financial crisis, average growth during 2008-09 is projected at $2 \frac{1}{2}$ percent, followed by an average growth of about $5 \frac{1}{2}$ percent during $2010-14$, and just above 6 percent during 2015-29. Although the assumed growth level is below that achieved in 2007, it is well above the pre-2003 historical average for Kenya. In addition to increasing investment, this higher growth potential results from significant progress made in addressing some long-standing weaknesses in public finance management, the financial sector, and the business climate (see IMF Country Report No. 08/339). There is a risk that constraints to growth-including infrastructure bottlenecks and the need for further improvements in the business environment - will prove binding in the medium-term. Accordingly, an additional scenario that assumes real growth for 2010-14 as one standard deviation lower than in the baseline scenario, is included (average real growth for 2010-14 in this scenario is just over $2 \frac{1}{2}$ percent).

- $\quad$ Average inflation of about 7 percent for 2009-14 as measured by a GDP deflator, which falls to 5 percent for the 2015-29 period.

- A constant real exchange rate is assumed during the medium term, with some appreciation in the longer run reflecting sustained high growth. Nominal exchange rates are from the World Economic Outlook (WEO).

- $\quad$ The non-interest external current account deficit (CAD) rises to about 6.3 percent of GDP in 2008, reflecting primarily higher oil prices before falling to an average of about 4 percent of GDP by 2014 and to only 0.9 percent of GDP during 2015-29 period. The improvement in the non-interest CAD reflects primarily the improvement in the trade deficit, which declines from almost 13 percent of GDP in 2008 to just over $3 \frac{1}{2}$ percent of GDP over the forecast period.

- $\quad$ Assumptions in the fiscal area include, broadly constant revenue as a share of GDP (about 21.5 percent); domestically financed development spending gradually increasing from just about 4.8 percent of GDP in 2008 to about 6.0 percent by 2029; a

\footnotetext{
${ }^{6}$ Public domestic debt includes central government debt. External public debt includes public and publicly guaranteed central government and central bank debt. In this analysis, total public debt refers to the sum of public domestic and public external debt, but does not cover the entire public sector (e.g., parastatal borrowing without a government guarantee is not covered).
} 
constant wage bill of 6.4 percent of GDP, and a gradual decline in other recurrent spending from 7.4 percent of GDP in 2008 to 6.0 percent of GDP over the long-term in line with the government's budget strategy. The primary fiscal deficit was 2.3 percent of GDP in 2008 and gradually declines to 1.0 by the end of the forecast period.

- Interest on short-term public domestic debt is assumed at 8 percent, whereas mediumand long-term domestic debt carry an assumed interest rate of about 10.5 percent. New domestic borrowings consist of a quarter of short-term debt and three-quarters of medium- and long-term debt, with the latter having an average maturity of about 7 years. The NPV of domestic debt is assumed to be equal to its face value.

- $\quad$ New external borrowing as a share of GDP (including nonconcessional borrowing described below) declines temporarily to 1 percent in 2008, from about 2 percent of GDP in 2007 before rising to an annual average of about $2 \frac{1}{2}$ percent during 2009-14, followed by a decline to just over $1 \frac{1}{2}$ percent by the end of the forecast period. In addition to borrowings from multilateral and bilateral creditors, new external borrowing assumptions include annual sovereign bond issuance of \$200 million during 2010-14, which is consistent with the authorities' medium-term budget framework. Annual external sovereign bond issuance would then rise beginning in 2015 to about $\$ 500$ million by 2029 . Assumptions on terms include an 8 percent interest rate and a bullet amortization in year ten (implying a nine year grace period on principal payments).

- $\quad$ Continued eligibility for concessional borrowing from IDA is assumed although achievement of assumed growth rates could imply graduation during the forecast period.

- $\quad$ The only debt relief assumed under the DSA is an existing swap agreement with Italy, which would cancel about Euro 44 million in external obligations in 2015.

\section{B. Outstanding Debt and Recent Developments}

\section{At end-2008, nominal public external debt was estimated at $\$ 6.2$ billion}

(20.4 percent of GDP). About 60 percent of this debt was to multilateral creditors (including 47 percent owed to the World Bank). About 39 percent of the debt was owed to bilateral creditors (largely the Paris Club), and a small share (under 2 percent) was owed to commercial creditors, some of which was for disputed security-related contracts, and in arrears (see para 8).

\section{Kenya has managed its debt burden relatively well and has regularly met its} obligations to most creditors. Limited external borrowing has left Kenya with more manageable debt ratios than many of its low-income country peers. Kenya's income level led it to be considered in the context of the Enhanced HIPC Initiative, but due to a debt levels 
below the HIPC Initiative thresholds, Kenya did not qualify to receive HIPC relief. ${ }^{7}$ Apart from disputed commercial arrears, described in greater detail in para 8, Kenya has regularly serviced its debts.

\section{Kenya's end-2008 external arrears of about $\$ 91$ million were to commercial} creditors and remain under dispute. The arrears stem from non-payment on commercial credits for security-related contracts, many of which have been found by Kenya's Controller and Auditor-General to be fraudulent or deeply flawed (these projects are often referred to as the "Anglo-Leasing" scandal). The authorities are disputing the validity of the claims based on the contracts not being fulfilled, have obtained an external audit to determine the value of the goods and services provided, and intend to renegotiate the amounts owed based on the completed audit results. Once agreement on the amounts to be paid is reached, it is expected that Kenya will refinance these payments through commercial bank loans initially, although part of the proceeds of the planned sovereign bond issuance could be used to finance arrears clearance.

\section{Kenya's net domestic debt stood at Ksh 340.4 billion at end-2008, or 16.1 percent} of GDP. Since 2003, domestic debt has gradually declined as percent of GDP thanks to strong economic growth, prudent fiscal policies, and lower interest rates, despite primary surpluses below historical averages and the realization of some one-off contingent liabilities in recent years. ${ }^{8}$ However, Kenya's relatively low reported domestic debt-to-GDP ratio masks vulnerabilities from further realization of contingent liabilities. In response, the government has launched a study of contingent liabilities in 24 parastatals and the National Social Security Fund. Results from this study will be incorporated into future debt sustainability analyses. In addition, the government's current pay-as-you-go pension scheme for civil servants has accumulated claims that were estimated at Ksh 271 billion (11.8 percent of 2008/09 GDP) in an August 2005 actuarial study. About 70 percent of Kenya's domestic debt is long-term borrowing with maturities extending up to 20 years. ${ }^{9}$

\section{External Debt Sustainability}

9. Under the baseline scenario, Kenya's external debt indicators show a low risk of debt distress. Kenya's initial debt ratios are well below all of the indicative thresholds for a medium performer and decline somewhat through the forecast period (see Figure 1 and Table 1a). Although external borrowing is projected to rise sharply over 2007-10, new obligations would fall gradually as a share of GDP thereafter. In addition to reasonably contained borrowing, the concessionality of new borrowing is slightly higher than in the past

\footnotetext{
${ }^{7}$ Kenya has benefited from Paris Club rescheduling.

${ }^{8}$ In 2006/07, the National Bank of Kenya was recapitalized and in 2007/08 contingent liabilities from Kenya Telkom were realized.

${ }^{9}$ Fiscal data, which are based on a July-June fiscal year, are converted to calendar year data for the purposes of the DSA. Averaging fiscal year data into calendar year data explains differences with Tables $2 \mathrm{a}$ and $2 \mathrm{~b}$ in the staff report.
} 
as shown in panel a of Figure 1), the improvement in the baseline scenario reflects favorable export growth and an improved trade balance projections in comparison to longer-term historical performance. Growth of both output and exports is projected to regain momentum once the global economy starts recovering from the ongoing financial crisis. Also, the infrastructure investments (financed partly through an assumed increase in external assistance) and structural reforms that the authorities are implementing are expected to boost productivity and support export and overall growth. It should be noted that the authorities' Kenya Vision 2030 targets much faster growth than assumed in the DSA.

10. The alternative scenarios and stress tests indicate that Kenya's external debt situation is generally resilient although shocks would lead to a sharp initial worsening in debt stock indicators (see Table 1b). Kenya would remain under its indicative debt stock and debt service thresholds under all alternative scenarios and stress tests. The most extreme stress tests are shown in Figure 1 and described here. Over the period 2010-14, a shock combining lower GDP growth, weaker exports, a lower GDP deflator, and a fall in non-debt creating flows would push the NPV of public external debt as a share of GDP from $14 \frac{1}{2}$ to 19 percent, the NPV of debt-to-exports from almost 57 to $671 \frac{1}{2}$ percent, and the NPV of debtto-revenue from 54 to 71 percent. Despite an immediate worsening, the three debt stock measures would then improve with ratios only somewhat worse than under the baseline scenario by the end of the projection period. The combined shock described above would also raise the debt service-to-exports and debt-service-to-revenue ratios. The scenario of lower growth for 2010-14 shows external debt indicators only marginally above those in the baseline scenario. The "historical" scenario, which uses the 10-year average performance of key economic variables instead of the baseline assumptions, yields debt ratios that are better than in the baseline scenario. The better debt profile reflects primarily the fact that the historical annual average of non-debt creating inflows exceeds the annual flows used in the baseline projections by almost $3 / 4$ percentage points of GDP a year. The ensuing reduction in borrowing needs leads to lower debt ratios than under the baseline scenario.

Summary -- External Debt Sustainability Assessment

(in percent of GDP)

\begin{tabular}{|c|c|c|c|c|c|c|}
\hline \multicolumn{7}{|c|}{$\begin{array}{c}\text { Summary -- External Debt Sustainability Assessment } \\
\text { (in percent of GDP) }\end{array}$} \\
\hline & 2009 & 2010 & 2011 & 2012 & 2013 & 2014 \\
\hline \multicolumn{7}{|l|}{ NPV of PPG External Debt } \\
\hline \multicolumn{7}{|c|}{ In percent of GDP (threshold $=40)$} \\
\hline Baseline & 11.8 & 11.2 & 10.6 & 10.9 & 11.2 & 11.5 \\
\hline Combined shocks & 11.8 & 14.6 & 18.6 & 18.6 & 18.9 & 19.1 \\
\hline \multicolumn{7}{|c|}{ In percent of exports (threshold $=150$ ) } \\
\hline Baseline & 47.9 & 51.0 & 50.5 & 51.5 & 50.9 & 51.0 \\
\hline Combined shocks & 47.9 & 56.9 & 69.6 & 69.6 & 67.6 & 66.6 \\
\hline \multicolumn{7}{|l|}{ PPG External Debt Service } \\
\hline \multicolumn{7}{|c|}{ In percent of exports $($ threshold $=20)$} \\
\hline Baseline & 3.5 & 3.3 & 3.3 & 3.2 & 3.1 & 2.9 \\
\hline Combined shocks & 3.5 & 3.4 & 3.7 & 3.9 & 3.7 & 3.4 \\
\hline
\end{tabular}




\section{Public Debt Sustainability}

11. Under the baseline scenario, the inclusion of domestic debt substantially increases the NPV of public debt-to-GDP (see Figure 2 and Table 2a). The NPV of total public debt-to-GDP, at 31.1 percent in 2008, remains broadly stable at that level before gradually trending down toward the end of the forecast period. Throughout the projection period, the NPV of total public debt remains below 35 percent under the baseline scenario. Given Kenya's relatively strong revenue performance, the NPV of debt-to-revenue ratio remains stays around 150 percent during the period 2009-2029. The debt service-to-revenue ratio, which includes debt service on longer-term domestic and external debt, increases to $26 \frac{1}{2}$ by 2012 from 25 percent in 2008 , and then gradually declines over time.

12. Alternative scenarios and stress tests indicate that Kenya's debt indicators are vulnerable to slower growth, while being broadly resilient under other alternative assumptions (see Figure 2 and Table 2b). A two-year growth shock leads to a rise in the NPV of debt-to-GDP ratio to 48 percent by 2029, an NPV of debt-to-revenue ratio to over 200 percent during the second half of the forecast period, and a rise in the debt service-torevenue ratio to around 30 percent by 2029. Permanently lower growth would also increase the NPV of debt-to-GDP beyond 40 percent, and would raise the debt service-to-revenue ratio close to 30 percent, even though the NPV of debt-to-revenue ratio would remain below 250 percent. The NPV of debt-to-GDP ratio also under a scenario with primary balances unchanged from 2009. The scenario of lower growth for 2010-14 results in debt indicators that are considerably higher in the long-term (e.g., by the end of the forecast period, the NPV of debt-to-GDP ratio would be 49 percent). This result reinforces the importance of expanding productive capacity in the medium term, in addition to a prudent borrowing approach, to avoid a rising debt burden.

\section{E. Conclusions}

\section{Reflecting the limited reliance on external borrowing and an expected} improvement in macroeconomic performance, Kenya faces a low risk of external debt distress. All external public debt indicators remain below the relevant country-specific debt burden thresholds. Standard stress tests reveal an initial upward trend for the debt indicators but do not result in a breach of the thresholds during the projection period.

14. Taking all public debt into account, however, the DSA shows greater risk of unfavorable debt developments, especially under a shock to GDP growth. Even temporarily lower GDP growth would set the NPV of public debt-to-GDP, the NPV of debtto-revenue, and the ratio of debt service-to-revenue on a sharply increasing trend as shown in Figure 2. Potentially large but unreported contingent liabilities also pose additional risks to the sustainability of public debt.

15. The sustainability of Kenya's debt depends on macroeconomic performance and a prudent borrowing strategy. Achieving the growth and export figures in the baseline scenario depends on: policies to sustain macroeconomic stability, substantial investment in infrastructure, and regulatory and governance reforms to improve the investment climate. 
Additionally, Kenya's success in avoiding unsustainable debt to date reflects good management, but also limited willingness on the part of creditors to provide financing, at times due to governance concerns. As Kenya is likely to have greater access to external financing in the future, it will be important to avoid excessive borrowing and to limit nonconcessional borrowing.

16. The staffs continue to encourage Kenya to use tools such as the joint Fund-Bank DSA template to develop a prudent borrowing strategy to maintain both external and fiscal debt sustainability. Efforts to use the DSA template and to develop a debt management strategy, including through a workshop in 2007 and the recent joint Bank-Fund technical assistance mission on the subject, are welcome. Such a strategy should consider the total concessionality and interest costs of Kenya's borrowing, maturity structure, and steps that would help guard against volatility, whether due to shocks such as droughts or to fluctuations in external assistance. Strategies to guard against shocks could include some further build-up in international reserves. A better understanding of holdings of shillingdenominated debt by non-residents would also help guide efforts to assess and reduce vulnerabilities. 
Figure 1. Kenya: Indicators of Public and Publicly Guaranteed External Debt under Alternatives Scenarios, 2009-2029 1/
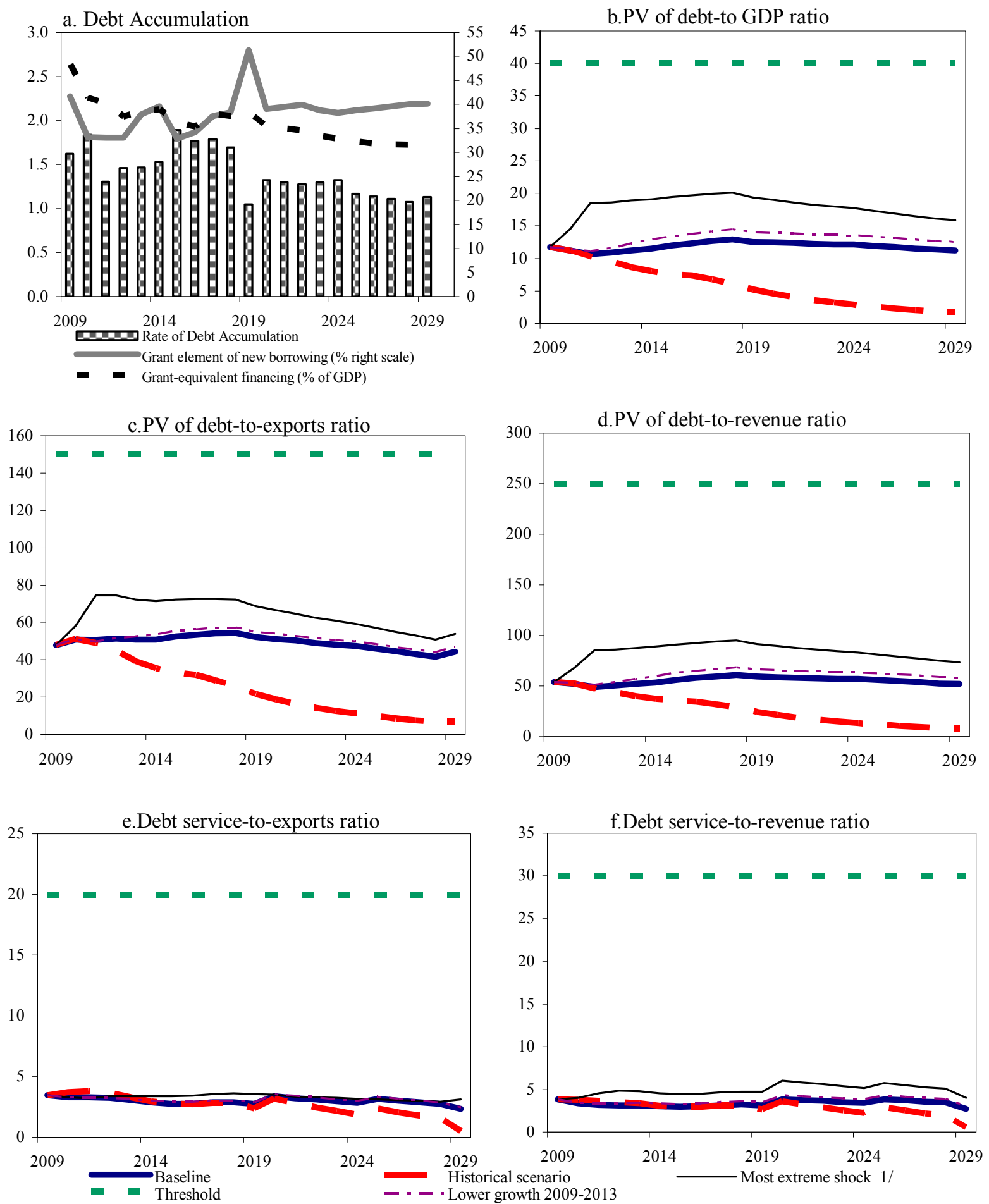

Sources: Country authorities and staff estimates and projections.

1/ The most extreme stress test is the test that yields the highest ratio in 2019. In figure b. it corresponds to a Combination shock; in c. to a Exports shock; in d. to a Combination shock; in e. to a Terms shock and in picture f. to a Combination shock. 
Figure 2. Kenya: Indicators of Public Debt Under Alternative Scenarios, 2009-2029 1/

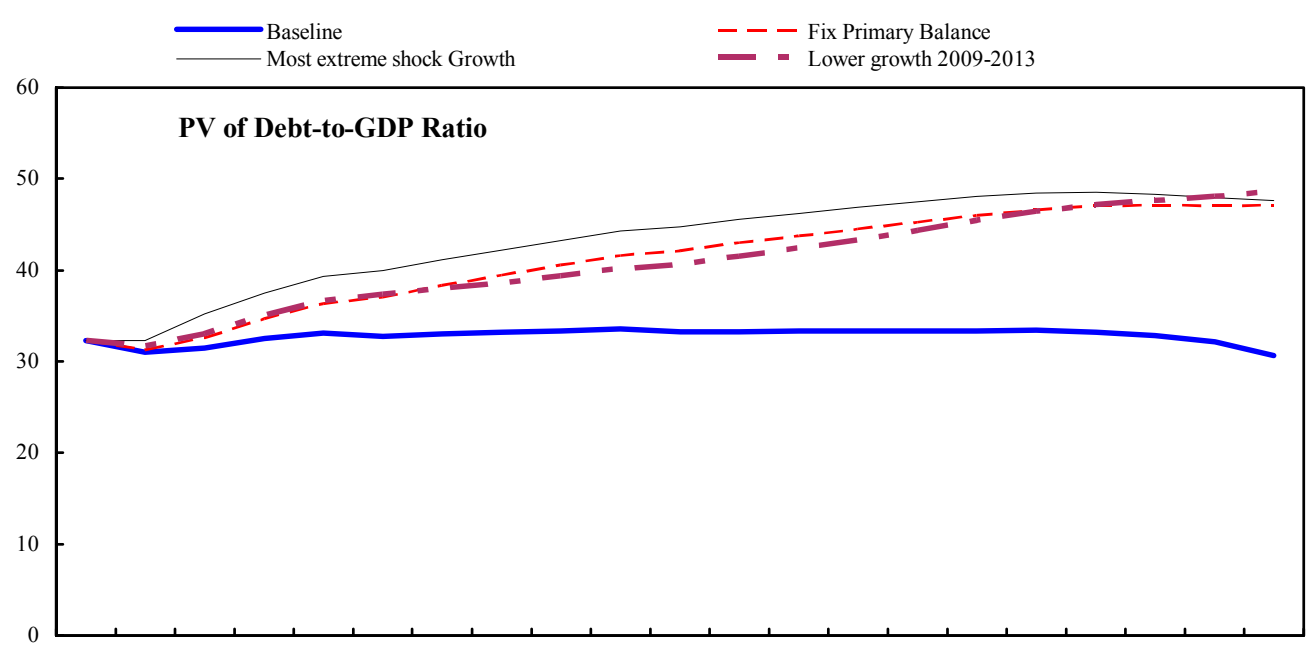

200920102011201220132014201520162017201820192020202120222023202420252026202720282029

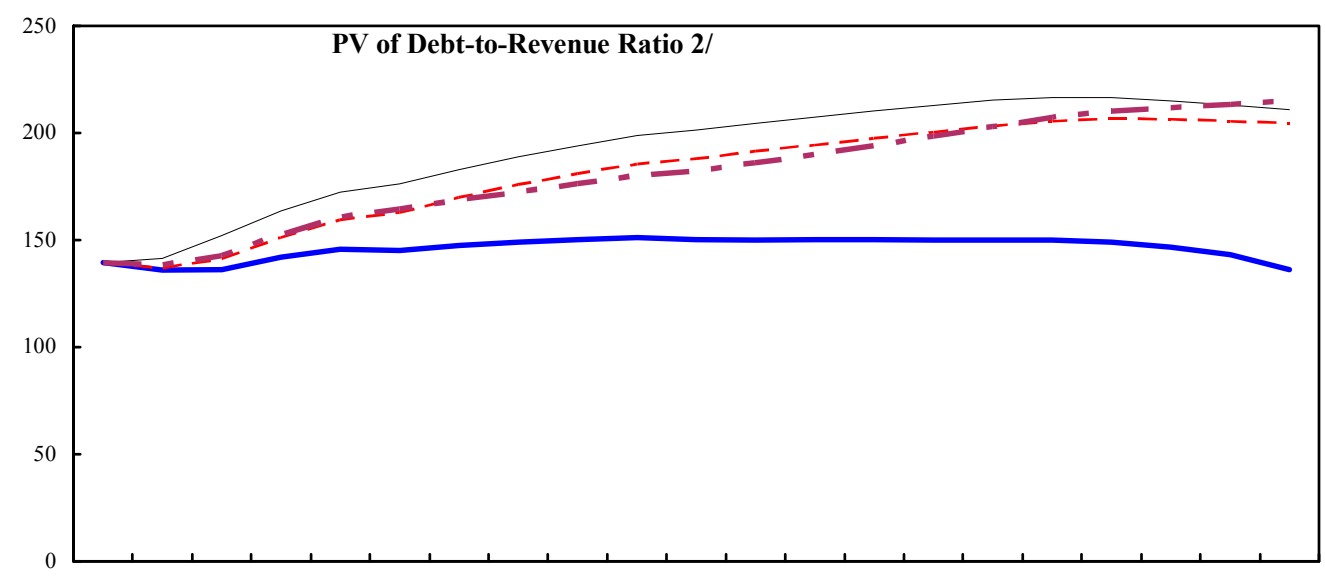

200920102011201220132014201520162017201820192020202120222023202420252026202720282029

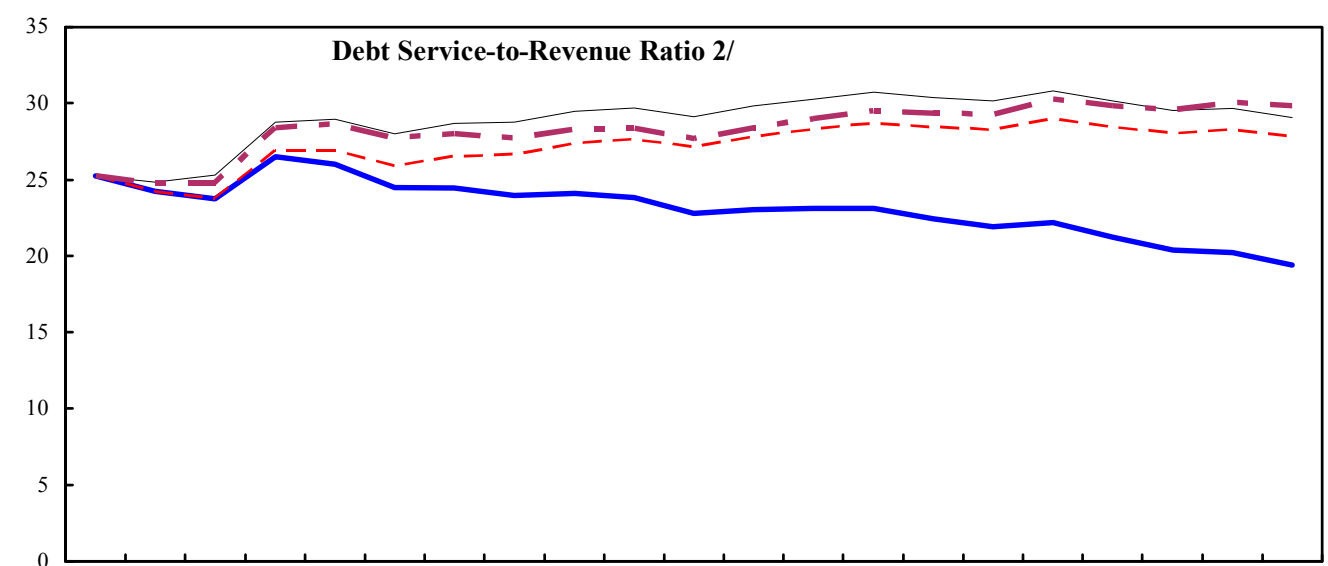

$20092010201120122013201420152016201720182019202020212022 \quad 2023202420252026202720282029$

Sources: Country authorities and staff estimates and projections.

$1 /$ The most extreme stress test is the test that yields the highest ratio in 2019.

2/ Revenues are defined inclusive of grants. 
Table 1a. Kenya: External Debt Sustainability Framework, Baseline Scenario, 2006-2029 1/

(In percent of GDP, unless otherwise indicated)

\begin{tabular}{|c|c|c|c|c|c|c|c|c|c|c|c|c|c|c|c|}
\hline & \multicolumn{3}{|c|}{ Actual } & \multirow{2}{*}{$\begin{array}{l}\text { Historical } \\
\text { Average 2/ }\end{array}$} & \multirow{2}{*}{$\begin{array}{c}\text { Standard } \\
\text { Deviation 2/ }\end{array}$} & \multicolumn{6}{|c|}{ Projections } & \multirow[b]{2}{*}{$\begin{array}{c}2009-2014 \\
\text { Average }\end{array}$} & \multirow[b]{2}{*}{2019} & \multirow[b]{2}{*}{2029} & \multirow[b]{2}{*}{$\begin{array}{c}2015-202 \\
\text { Average }\end{array}$} \\
\hline & 2006 & 2007 & 2008 & & & 2009 & 2010 & 2011 & 2012 & 2013 & 2014 & & & & \\
\hline External debt (nominal) 1/ & 26.8 & 24.7 & 22.0 & & & 24.7 & 22.2 & 20.8 & 20.7 & 21.0 & 21.0 & & 21.0 & 18.0 & \\
\hline $\mathrm{o} / \mathrm{w}$ public and publicly guaranteed (PPG) & 24.9 & 22.8 & 20.4 & & & 23.3 & 21.2 & 19.6 & 19.5 & 19.8 & 20.0 & & 20.6 & 18.0 & \\
\hline Change in external debt & -4.5 & -2.1 & -2.8 & & & 2.7 & -2.5 & -1.5 & -0.1 & 0.3 & 0.0 & & -0.4 & -0.3 & \\
\hline Identified net debt-creating flows & -5.0 & -4.0 & 1.9 & & & 1.4 & 2.7 & 2.3 & 2.0 & 1.3 & 1.0 & & -0.1 & -1.1 & \\
\hline Non-interest current account deficit & 2.0 & 3.7 & 6.3 & 1.1 & 3.1 & 3.4 & 4.6 & 4.5 & 4.3 & 3.6 & 3.3 & & 1.1 & -0.2 & 0.9 \\
\hline Deficit in balance of goods and services & 9.3 & 10.1 & 12.9 & & & $\begin{array}{r}3.4 \\
10.2\end{array}$ & 10.1 & 9.5 & 9.1 & 8.5 & 8.2 & & 5.7 & 3.6 & \\
\hline Exports & 25.9 & 26.1 & 27.2 & & & 24.6 & 21.9 & 21.0 & 21.1 & 22.1 & 22.6 & & 24.0 & 25.2 & \\
\hline Imports & 35.1 & 36.2 & 40.2 & & & 34.8 & 32.0 & 30.5 & 30.3 & 30.5 & 30.8 & & 29.6 & 28.8 & \\
\hline Net current transfers (negative $=$ inflow) & -7.0 & -6.4 & -6.3 & -6.1 & 1.0 & -6.5 & -5.4 & -4.9 & -4.7 & -4.8 & -4.7 & & -4.4 & -3.5 & -4.2 \\
\hline o/w official 3/ & -0.3 & -0.2 & 0.0 & & & 0.1 & 0.1 & 0.1 & 0.0 & 0.0 & 0.0 & & 0.0 & 0.0 & \\
\hline Other current account flows (negative $=$ net inflow) & -0.3 & 0.0 & -0.3 & & & -0.2 & -0.1 & -0.1 & -0.1 & -0.1 & -0.1 & & -0.2 & -0.3 & \\
\hline Net FDI (negative $=$ inflow) & -2.2 & -3.6 & -2.2 & -1.2 & 1.1 & -1.6 & -1.3 & -1.4 & -1.4 & -1.4 & -1.4 & & $\begin{array}{lll}-0.2 \\
-0.5\end{array}$ & -0.2 & -0.4 \\
\hline Endogenous debt dynamics 4 / & -4.7 & -4.0 & -2.2 & & & -0.4 & -0.6 & -0.7 & -0.9 & -0.9 & -0.9 & & -0.8 & -0.7 & \\
\hline Contribution from nominal interest rate & 0.5 & 0.4 & 0.4 & & & 0.3 & 0.2 & 0.2 & 0.3 & 0.3 & 0.3 & & 0.4 & 0.3 & \\
\hline Contribution from real GDP growth & -1.7 & -1.6 & -0.4 & & & -0.7 & -0.8 & -0.9 & -1.2 & -1.2 & -1.2 & & -1.2 & -1.0 & \\
\hline Contribution from price and exchange rate changes & -3.5 & -2.9 & -2.2 & & & & & & & & & & & & \\
\hline Residual (3-4) 5/ & 0.5 & 1.9 & -4.6 & & & 1.3 & -5.2 & -3.8 & -2.1 & $\begin{array}{r}-0.9 \\
-10\end{array}$ & -1.0 & & -0.2 & 0.8 & \\
\hline $\mathrm{o} / \mathrm{w}$ exceptional financing & -1.1 & 0.0 & 0.0 & & & -0.1 & 0.0 & 0.0 & 0.0 & 0.0 & 0.0 & & 0.0 & 0.0 & \\
\hline PV of external debt 6/ & $\ldots$ & $\ldots$ & 11.6 & & & 13.2 & 12.2 & 11.8 & 12.1 & 12.5 & 12.5 & & 12.8 & 11.2 & \\
\hline In percent of exports & $\ldots$ & $\ldots$ & 42.6 & & & 53.7 & 55.8 & 55.8 & 57.2 & 56.6 & 55.4 & & 53.6 & 44.5 & \\
\hline PV of PPG external debt & $\ldots$ & $\ldots$ & 10.0 & & & 11.8 & 11.2 & 10.6 & 10.9 & 11.2 & 11.5 & & 12.5 & 11.2 & \\
\hline In percent of exports & $\ldots$ & $\ldots$ & 36.7 & & & 47.9 & $\mathbf{5 1 . 0}$ & 50.5 & 51.5 & 50.9 & 51.0 & & 52.3 & 44.5 & \\
\hline In percent of government revenues & $\ldots$ & $\ldots$ & 45.0 & & & 53.8 & 52.1 & 49.1 & 50.3 & 52.1 & 53.7 & & 59.3 & 52.0 & \\
\hline Debt service-to-exports ratio (in percent) & 6.5 & 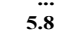 & 5.1 & & & 4.5 & 4.2 & 4.0 & 3.8 & 3.7 & 3.5 & & 2.9 & 2.3 & \\
\hline PPG debt service-to-exports ratio (in percent) & 5.5 & 4.8 & 4.2 & & & 3.5 & 3.3 & 3.3 & 3.2 & 3.1 & 2.9 & & 2.7 & 2.3 & \\
\hline PPG debt service-to-revenue ratio (in percent) & 6.7 & 5.6 & 5.1 & & & 3.9 & 3.4 & 3.2 & 3.2 & 3.1 & 3.0 & & 3.1 & 2.7 & \\
\hline Total gross financing need (billions of U.S. dollars) & 0.3 & 0.4 & 1.7 & & & 0.9 & 1.6 & 1.7 & 1.8 & 1.6 & 1.6 & & 1.3 & 0.5 & \\
\hline Non-interest current account deficit that stabilizes debt ratio & 6.5 & 5.7 & 9.0 & & & 0.6 & 7.1 & 5.9 & 4.4 & 3.3 & 3.3 & & 1.5 & 0.1 & \\
\hline \multicolumn{16}{|l|}{ Key macroeconomic assumptions } \\
\hline Real GDP growth (in percent) & 6.4 & 7.0 & 2.0 & 3.7 & 2.4 & 3.0 & 4.0 & 5.0 & 6.3 & 6.5 & 6.5 & 5.2 & 6.0 & 6.0 & 6.1 \\
\hline GDP deflator in US dollar terms (change in percent) & 12.8 & 12.2 & 9.7 & 4.8 & 9.0 & -4.4 & 17.2 & 11.9 & 4.8 & 3.1 & 3.9 & 6.1 & 5.0 & 5.0 & 5.0 \\
\hline Effective interest rate (percent) $7 /$ & 2.0 & 2.0 & 1.7 & 1.8 & 1.0 & 1.4 & 1.2 & 1.3 & 1.4 & 1.5 & 1.6 & 1.4 & 2.0 & 1.8 & 1.9 \\
\hline Growth of exports of G\&S (US dollar terms, in percent) & 9.2 & 21.3 & 16.5 & $\begin{array}{r}1.8 \\
11.6\end{array}$ & 9.2 & $\begin{array}{r}1.4 \\
-10.9\end{array}$ & $\begin{array}{l}1.2 \\
8.5\end{array}$ & $\begin{array}{r}1.3 \\
12.8\end{array}$ & $\begin{array}{l}1.4 \\
11.6\end{array}$ & 14.8 & $\begin{array}{r}1.6 \\
13.4\end{array}$ & $\begin{array}{l}1.4 \\
8.4\end{array}$ & $\begin{array}{r}2.0 \\
12.7\end{array}$ & $\begin{array}{l}1.8 \\
3.3\end{array}$ & 12.2 \\
\hline Growth of imports of G\&S (US dollar terms, in percent) & 17.4 & 23.6 & 24.1 & 13.8 & 16.8 & -14.7 & 12.0 & 12.1 & 10.4 & 10.8 & 11.6 & 7.0 & 11.6 & 3.0 & 10.9 \\
\hline Grant element of new public sector borrowing & & & & $\ldots$ & $\ldots$ & 41.8 & 33.2 & 33.1 & 33.1 & 38.0 & 39.6 & 36.4 & 51.3 & 40.2 & 39.2 \\
\hline Government revenues (excluding grants, in percent of GDP) & 21.1 & 22.2 & 22.2 & & & 21.9 & 21.5 & 21.7 & 21.6 & 21.5 & 21.5 & & 21.1 & 21.5 & 21.3 \\
\hline Aid flows (in billions of US dollars) 8 ; & 0.2 & 0.3 & 0.4 & & & 1.4 & 2.3 & 3.2 & 4.0 & 5.1 & 6.4 & & 15.1 & 47.9 & \\
\hline o/w Grants & 0.2 & 0.3 & 0.4 & & & 0.4 & 0.5 & 0.6 & 0.6 & 0.6 & 0.7 & & 1.0 & 2.8 & \\
\hline o/w Concessional loans & 0.0 & 0.0 & 0.0 & & & 1.0 & 1.8 & 2.5 & 3.4 & 4.5 & 5.7 & & 14.1 & 45.1 & \\
\hline Grant-equivalent financing (in percent of GDP) $9 /$ & $\ldots$ & $\ldots$ & $\ldots$ & & & 2.6 & 2.3 & 2.2 & 2.0 & 2.1 & 2.1 & & 2.1 & 1.7 & 1.9 \\
\hline Grant-equivalent financing (in percent of external financing) $9 /$ & $\ldots$ & $\ldots$ & $\ldots$ & & & 57.4 & 56.2 & 58.5 & 57.4 & 58.0 & 58.3 & & 67.2 & 60.7 & 57.5 \\
\hline Memorandum items: & & & & & & & & & & & & & & & \\
\hline Nominal GDP (billions of US dollars) & 22.5 & 27.0 & 30.2 & & & 29.8 & 36.3 & 42.6 & 47.4 & 52.1 & 57.6 & & 99.5 & 290.4 & \\
\hline Nominal dollar GDP growth & 20.0 & 20.0 & 11.9 & & & -1.5 & 21.9 & 17.5 & 11.3 & 9.8 & 10.6 & 11.6 & 11.3 & 11.3 & 11.4 \\
\hline PV of PPG external debt (in billions of US dollars) & & & 3.0 & & & 3.5 & $\begin{array}{r}2.99 \\
4.1\end{array}$ & 4.5 & 5.2 & $\begin{array}{l}9.8 \\
5.9\end{array}$ & 6.6 & & 12.5 & 32.5 & 11.4 \\
\hline (PVt-PVt-1)/GDPt-1 (in percent) & & & & & & 1.6 & 1.8 & 1.3 & 1.5 & 1.5 & 1.5 & 1.5 & 1.0 & 1.1 & 1.4 \\
\hline
\end{tabular}

Sources: Country authorities and staff estimates and projections.

$1 /$ Includes both public and private sector external debt.

2/ Historical averages and standard deviations are generally derived over the past 10 years, subject to data availability.

3/ Includes only program grants.

Derived as $[\mathrm{r}-\mathrm{g}-\mathrm{r}(\mathrm{l}+\mathrm{g})](\mathrm{i}+\mathrm{g}+\mathrm{r}+\mathrm{gr})$ times previous period debt ratio, with $\mathrm{r}=$ nominal interest rate; $\mathrm{g}=$ real GDP growth rate, and $\mathrm{r}=$ growth rate of GDP deflator in U.S. dollar terms.

5/ Includes exceptional financing (i.e., changes in arrears and debt relief); changes in gross foreign assets; and valuation adjustments. For projections also includes contribution from price and exchange rate changes.

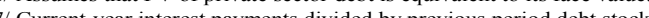

Defined as gants (program and project), concescionl loans, and debtelief.

9/ Grant-equivalent financing includes grants provided directly to the government and through new borrowing (difference between the face value and the PV of new debt). 
Table 1b. Kenya: Sensitivity Analysis for Key Indicators of Public and Publicly Guaranteed External Debt, 2009-2029 (In percent)

\begin{tabular}{|c|c|c|c|c|c|c|c|c|c|c|c|c|c|c|c|c|c|c|c|c|c|}
\hline & \multicolumn{21}{|c|}{ Projections } \\
\hline & 2009 & 2010 & 2011 & 2012 & 2013 & 2014 & 2015 & 2016 & 2017 & 2018 & 2019 & 2020 & 2021 & 2022 & 2023 & 2024 & 2025 & 2026 & 2027 & 2028 & 2029 \\
\hline \multicolumn{22}{|c|}{ PV of debt-to GDP ratio } \\
\hline Baseline & 12 & 11 & 11 & 11 & 11 & 12 & 12 & 12 & 13 & 13 & 13 & 12 & 12 & 12 & 12 & 12 & 12 & 12 & 12 & 11 & 11 \\
\hline \multicolumn{22}{|l|}{ A. Alternative Scenarios } \\
\hline A1. Key variables at their historical averages in 2009-2029 1/ & 12 & 11 & 10 & 10 & 9 & 8 & 8 & 7 & 7 & 6 & 5 & 5 & 4 & 4 & 3 & 3 & 3 & 2 & 2 & 2 & \\
\hline $\begin{array}{l}\text { A2. New public sector loans on less favorable terms in } 2009-202921 \\
\text { A3. Alternative Scenario: Growth lower by one standard deviation 2010-2014 }\end{array}$ & $\begin{array}{l}12 \\
12\end{array}$ & $\begin{array}{l}11 \\
11\end{array}$ & $\begin{array}{l}11 \\
11\end{array}$ & $\begin{array}{l}12 \\
12\end{array}$ & $\begin{array}{l}13 \\
12\end{array}$ & $\begin{array}{l}13 \\
13\end{array}$ & $\begin{array}{l}14 \\
13\end{array}$ & $\begin{array}{l}15 \\
14\end{array}$ & $\begin{array}{l}15 \\
14\end{array}$ & $\begin{array}{l}16 \\
14\end{array}$ & $\begin{array}{l}\mathbf{1 6} \\
14\end{array}$ & $\begin{array}{l}16 \\
14\end{array}$ & $\begin{array}{l}16 \\
14\end{array}$ & $\begin{array}{l}16 \\
14\end{array}$ & $\begin{array}{l}16 \\
14\end{array}$ & $\begin{array}{l}16 \\
14\end{array}$ & $\begin{array}{l}16 \\
13\end{array}$ & $\begin{array}{l}16 \\
13\end{array}$ & $\begin{array}{l}16 \\
13\end{array}$ & $\begin{array}{l}15 \\
13\end{array}$ & $\begin{array}{l}15 \\
13\end{array}$ \\
\hline \multicolumn{22}{|l|}{ B. Bound Tests } \\
\hline B1. Real GDP growth at historical average minus one standard deviation in 2010-2011 & 12 & 11 & 11 & 12 & 12 & 12 & 13 & 13 & 13 & 14 & 13 & 13 & 13 & 13 & 13 & 13 & 13 & 12 & 12 & 12 & \\
\hline B2. Export value growth at historical average minus one standard deviation in 2010-2011 3/ & 12 & 12 & 13 & 13 & 14 & 14 & 14 & 14 & 15 & 15 & 14 & 14 & 14 & 13 & 13 & 13 & 13 & 12 & 12 & 12 & 12 \\
\hline 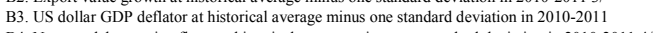 & 12 & 14 & 15 & 16 & 16 & 16 & 17 & 18 & 18 & 18 & 18 & 18 & 18 & 17 & 17 & 17 & 17 & 17 & 17 & 16 & 16 \\
\hline 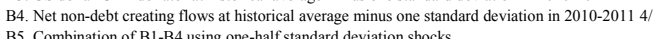 & 12 & 12 & 12 & 12 & 13 & $\begin{array}{l}13 \\
19\end{array}$ & 13 & $\begin{array}{l}13 \\
20\end{array}$ & $\begin{array}{l}14 \\
20\end{array}$ & $\begin{array}{l}14 \\
20\end{array}$ & 13 & 13 & 13 & ${ }_{18}^{13}$ & 13 & 13 & 12 & 12 & 12 & 12 & 11 \\
\hline $\begin{array}{l}\text { B5. Combination of B1-B4 using one-half standard deviation shocks } \\
\text { B6. One-time } 30 \text { percent nominal depreciation relative to the baseline in } 20105 /\end{array}$ & $\begin{array}{l}12 \\
12\end{array}$ & $\begin{array}{l}15 \\
15\end{array}$ & ${ }_{14}^{19}$ & $\begin{array}{l}199 \\
15\end{array}$ & $\begin{array}{l}19 \\
15\end{array}$ & $\begin{array}{l}19 \\
16\end{array}$ & $\begin{array}{l}19 \\
16\end{array}$ & $\begin{array}{l}20 \\
17\end{array}$ & $\begin{array}{l}20 \\
17\end{array}$ & $\begin{array}{l}20 \\
17\end{array}$ & $\begin{array}{l}19 \\
17\end{array}$ & $\begin{array}{l}19 \\
17\end{array}$ & $\begin{array}{l}19 \\
17\end{array}$ & $\begin{array}{l}18 \\
16\end{array}$ & $\begin{array}{l}18 \\
16\end{array}$ & $\begin{array}{l}18 \\
16\end{array}$ & $\begin{array}{l}17 \\
16\end{array}$ & $\begin{array}{l}17 \\
16\end{array}$ & $\begin{array}{l}17 \\
16\end{array}$ & $\begin{array}{l}16 \\
15\end{array}$ & $\begin{array}{l}16 \\
15\end{array}$ \\
\hline \multicolumn{22}{|c|}{ PV of debt-to-exports ratio } \\
\hline Baseline & 48 & 51 & 51 & 51 & 51 & 51 & 53 & 53 & 54 & 54 & 52 & 51 & 50 & 49 & 48 & 47 & 46 & 45 & 43 & 42 & 44 \\
\hline \multicolumn{22}{|l|}{ A. Alternative Scenarios } \\
\hline A1. Key variables at their historical averages in 2009-2029 1/ & 48 & 51 & 49 & 45 & 39 & 35 & 33 & 32 & 29 & 26 & 22 & 19 & 17 & 14 & 13 & 11 & 10 & 9 & 8 & 7 & 7 \\
\hline sector loans on les: & 48 & 51 & 52 & 55 & 57 & 58 & 60 & 63 & 66 & 67 & 67 & 64 & 64 & 63 & 62 & 62 & 61 & 60 & 58 & 57 & 60 \\
\hline A3. Alternative Scenario: Growth lower by one standard deviation 2010-2014 & 48 & 51 & 50 & 52 & 52 & 54 & 55 & 56 & 57 & 57 & 55 & 54 & 53 & 52 & 51 & 50 & 48 & 47 & 45 & 44 & 47 \\
\hline \multicolumn{22}{|l|}{ B. Bound Tests } \\
\hline B1. Real GDP growh at historical average minus one standard deviation in 2010-2011 & 48 & 51 & 51 & 51 & 51 & 51 & 53 & 53 & 54 & 54 & 52 & 51 & 50 & 49 & 48 & 47 & 46 & 45 & 43 & 42 & 44 \\
\hline B2. Export value grow & 48 & 58 & 74 & 74 & 72 & 71 & 72 & 72 & 73 & 72 & 69 & 67 & 65 & 63 & 61 & 59 & 57 & 55 & 53 & 51 & 54 \\
\hline B3. US dollar GDP deflator at historical average minus one standard deviation in 2010-2011 & 48 & 511 & 51 & $\begin{array}{c}51 \\
58\end{array}$ & 51 & $\begin{array}{c}51 \\
57\end{array}$ & 53 & 53 & 54 & 54 & 52 & 51 & 50 & 49 & 48 & 47 & 46 & 45 & 43 & 42 & ${ }_{45}^{44}$ \\
\hline $\begin{array}{l}\text { B4. Net non-debt creating fllows at historiacal average minus one standard deviation in 2010-2011 4/ } \\
\text { B5 Combination of B-1-44 using one-half standard deviation shocks }\end{array}$ & $\begin{array}{l}48 \\
48\end{array}$ & $\begin{array}{l}55 \\
57\end{array}$ & $\begin{array}{l}58 \\
70\end{array}$ & $\begin{array}{l}58 \\
70\end{array}$ & $\begin{array}{l}57 \\
68\end{array}$ & $\begin{array}{l}57 \\
67\end{array}$ & $\begin{array}{l}58 \\
67\end{array}$ & $\begin{array}{l}58 \\
67\end{array}$ & $\begin{array}{l}59 \\
67\end{array}$ & $\begin{array}{l}59 \\
67\end{array}$ & $\begin{array}{l}56 \\
64\end{array}$ & $\begin{array}{l}55 \\
62\end{array}$ & 53 & $\begin{array}{l}52 \\
58\end{array}$ & $\begin{array}{l}50 \\
56\end{array}$ & $\begin{array}{l}49 \\
55\end{array}$ & ${ }_{53}^{48}$ & $\begin{array}{l}46 \\
46\end{array}$ & $\begin{array}{l}44 \\
49\end{array}$ & ${ }_{47}^{43}$ & $\begin{array}{l}45 \\
50\end{array}$ \\
\hline 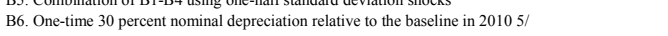 & 48 & 51 & (51 & ${ }_{51}^{10}$ & $\begin{array}{l}68 \\
51\end{array}$ & ${ }_{51}^{01}$ & 53 & 53 & 54 & $\begin{array}{l}61 \\
54\end{array}$ & 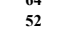 & $\begin{array}{l}{ }_{51}^{22} \\
51\end{array}$ & ${ }_{50}^{0}$ & $\begin{array}{l}88 \\
49\end{array}$ & 48 & $\begin{array}{l}33 \\
47\end{array}$ & 46 & $\begin{array}{l}51 \\
45\end{array}$ & 43 & 42 & 年 \\
\hline \multicolumn{22}{|c|}{ PV of debt-to-revenue ratio } \\
\hline Baseline & 54 & 52 & 49 & 50 & 52 & 54 & 56 & 58 & 60 & 61 & 59 & 59 & 58 & 58 & 57 & 57 & 56 & 55 & 54 & 53 & 52 \\
\hline \multicolumn{22}{|l|}{ A. Alternative Scenarios } \\
\hline A1. Key variables at their historical av & 54 & 52 & 48 & 44 & 40 & 37 & 36 & 35 & 32 & 29 & 25 & 22 & 19 & 17 & 15 & 14 & 12 & 11 & 10 & 9 & 8 \\
\hline $\begin{array}{l}\text { A2. New public sector loans on less favorable terms in } 2009-20292 / \\
\text { A3. Alternative Scenario: Growth lower by one standard deviation 2010-2014 }\end{array}$ & $\begin{array}{l}54 \\
54\end{array}$ & $\begin{array}{l}52 \\
53\end{array}$ & $\begin{array}{c}51 \\
51\end{array}$ & $\begin{array}{l}54 \\
54\end{array}$ & $\begin{array}{l}58 \\
57\end{array}$ & $\begin{array}{l}61 \\
60\end{array}$ & $\begin{array}{l}64 \\
63\end{array}$ & $\begin{array}{l}68 \\
65\end{array}$ & $\begin{array}{l}72 \\
67\end{array}$ & $\begin{array}{l}75 \\
68\end{array}$ & $\begin{array}{l}75 \\
66\end{array}$ & $\begin{array}{l}74 \\
66\end{array}$ & $\begin{array}{l}74 \\
65\end{array}$ & $\begin{array}{l}74 \\
65\end{array}$ & $\begin{array}{l}74 \\
64\end{array}$ & $\begin{array}{l}74 \\
64\end{array}$ & $\begin{array}{r}74 \\
63\end{array}$ & $\begin{array}{l}73 \\
61\end{array}$ & $\begin{array}{l}73 \\
60\end{array}$ & $\begin{array}{l}72 \\
59\end{array}$ & $\begin{array}{c}71 \\
58\end{array}$ \\
\hline \multicolumn{22}{|l|}{ B. Bound Tests } \\
\hline B1. Real GDP growth at hi & 54 & & & & & 57 & 60 & & & 65 & 63 & & & 61 & 61 & & & 58 & 57 & 56 & \\
\hline B2. Export value growh at histo & 54 & 56 & 62 & 62 & 64 & 64 & 66 & 67 & 69 & 69 & 67 & 65 & 64 & 63 & 62 & 61 & 59 & 58 & 57 & 55 & 54 \\
\hline B3. US dollar GDP deffator at historical average minus one standard deviation in 2010-2011 & 54 & 64 & 70 & 72 & 75 & 77 & 80 & 83 & 85 & 87 & 85 & 84 & 83 & 82 & 82 & 81 & 80 & 78 & 77 & 75 & $\begin{array}{l}74 \\
53\end{array}$ \\
\hline $\begin{array}{l}\text { B4. Net non-debt creating flows at historicalaverage minus one standard deviation in 2010-2011 4/ } \\
\text { B5. Combination of B1-B4 using one-half standard deviation shocks }\end{array}$ & 54 & $\begin{array}{l}57 \\
68\end{array}$ & $\begin{array}{l}56 \\
86\end{array}$ & $\begin{array}{l}57 \\
86\end{array}$ & $\begin{array}{l}59 \\
88\end{array}$ & $\begin{array}{l}60 \\
89\end{array}$ & $\begin{array}{l}62 \\
91\end{array}$ & $\begin{array}{l}63 \\
93\end{array}$ & $\begin{array}{l}65 \\
94\end{array}$ & $\begin{array}{l}66 \\
95\end{array}$ & $\begin{array}{l}64 \\
92\end{array}$ & $\begin{array}{l}63 \\
90\end{array}$ & $\begin{array}{l}62 \\
88\end{array}$ & $\begin{array}{l}61 \\
86\end{array}$ & $\begin{array}{l}60 \\
84\end{array}$ & $\begin{array}{l}59 \\
83\end{array}$ & $\begin{array}{l}58 \\
81\end{array}$ & $\begin{array}{l}57 \\
79\end{array}$ & $\begin{array}{l}55 \\
77\end{array}$ & $\begin{array}{l}54 \\
75\end{array}$ & $\begin{array}{l}53 \\
74\end{array}$ \\
\hline 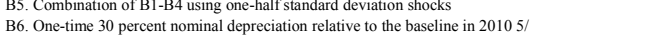 & 54 & ${ }_{70}^{68}$ & $\begin{array}{l}86 \\
66\end{array}$ & 68 & $\begin{array}{l}88 \\
70\end{array}$ & $\begin{array}{l}89 \\
72\end{array}$ & $\begin{array}{l}91 \\
76\end{array}$ & ${ }_{78}^{93}$ & $\begin{array}{l}94 \\
80\end{array}$ & ${ }_{82}^{95}$ & 92 & $\begin{array}{l}90 \\
79\end{array}$ & ${ }_{78}^{88}$ & $\begin{array}{l}86 \\
77\end{array}$ & ${ }_{77}^{84}$ & ${ }_{76}^{83}$ & $\begin{array}{l}81 \\
75\end{array}$ & 74 & 72 & 71 & \\
\hline
\end{tabular}


Table 1b. Kenya: Sensitivity Analysis for Key Indicators of Public and Publicly Guaranteed External Debt, $2009-2029$ (continued)

(In percent)

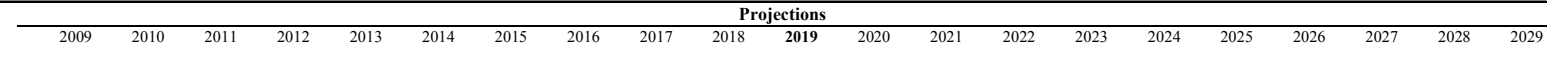

Debt service-to-exports ratio

Baseline

A. Alternative Scenarios

A1. Key variables at their historical averages in 2009-2029 $1 /$

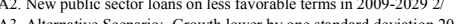

B. Bound Tests

B1. Real GDP growth at historical average minus one standard deviation in 2010-2011

B2. Export value growth at historical average minus one standard deviation in 2010-2011 3/
B3. US dollar GDP deflator at historical average minus one standard deviation in 2010-2011
B4. Net non-debt creating flows at historical average minus one standard deviation in $2010-2011$

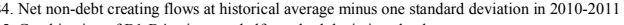

B6. One-time 30 percent nominal depreciation relative to the baseline in $20105 /$

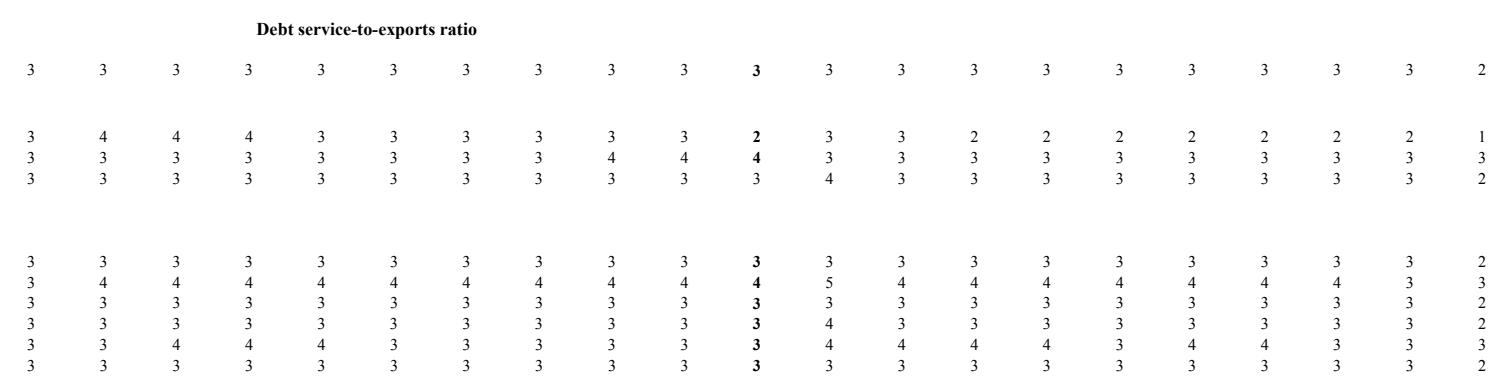

Baseline

A. Alternative Scenarios

A1. Key variables at their historical averages in 2009-2029 $1 /$

A3. Ale

B. Bound Tests

B1. Real GDP growth at historical average minus one standard deviation in 2010-2011-

B2. Export value growh at historical average minus one standard deviation in 2010-20113/

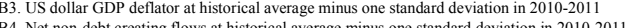

B5. Net non-

B6. One-time 30 percent nominal depreciation relative to the baseline in $20105 /$

Debt service-to-revenue ratio

Memorandum item:
Grant element assumed on residual financing (i.e., financing required above baseline) $6 /$

Sources: Country authorities and staff estimates and projections

1/ Variables include real GDP growth, growth of GDP defflator (in U.S. dollar terms), non-interest current account in percent of GDP, and non-debt creating flows.

is assumed to return to its baseline level after the shock (implicitly assuming

an offeetting adjustment in import levels).
$4 /$ Includes official and private transfers and FDI.

(1)

except for A2 (less favorable financing) in which the terms on all new financing are as specified in footnote 2. 
Table 2a. Kenya: Public Sector Debt Sustainability Framework, Baseline Scenario, 2006-2029

(In percent of GDP, unless otherwise indicated)

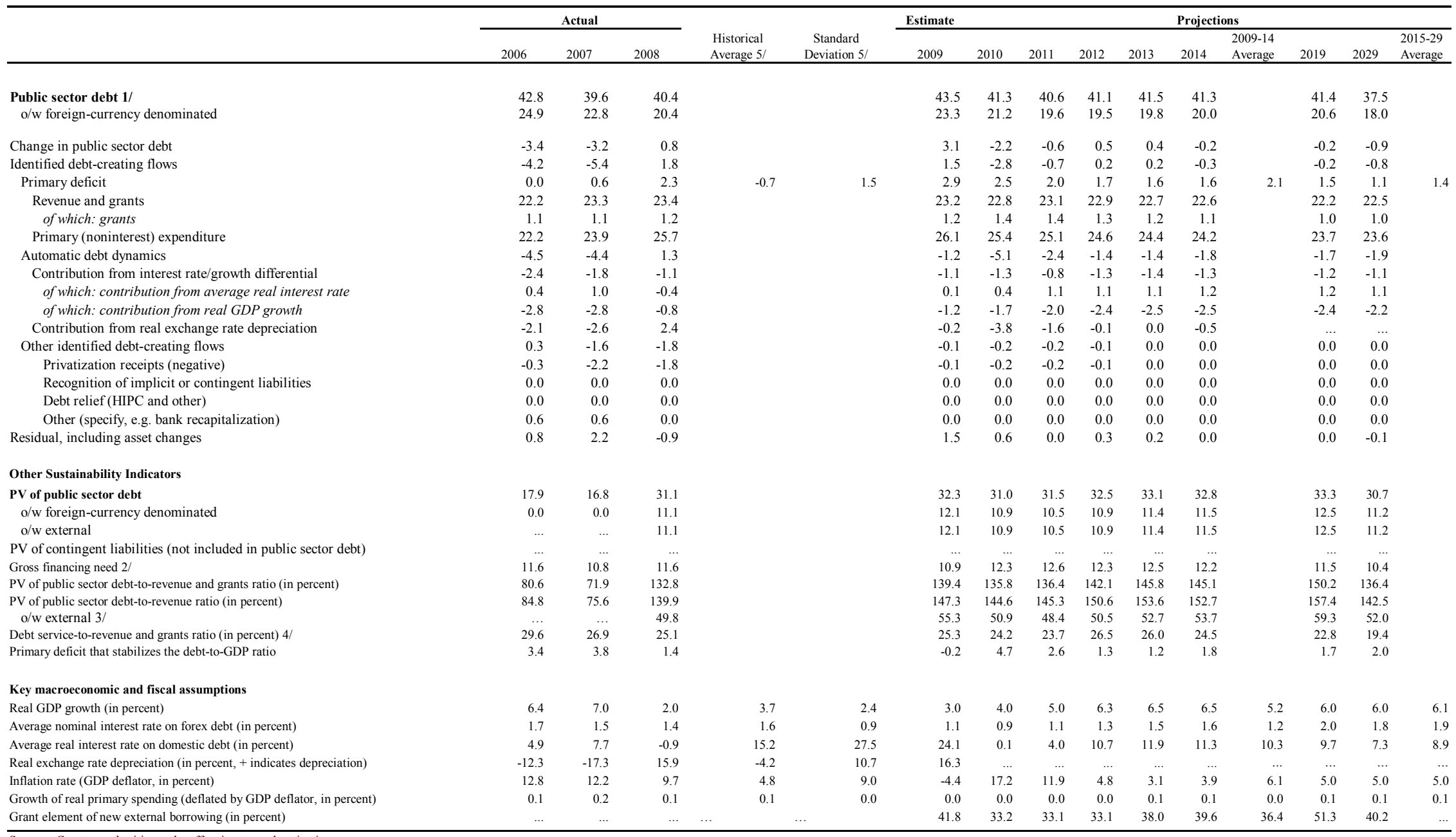

debt and external debt issued or guaranteed by the central government and central bank.

2/ Gross financing need is defined as the primary deficit plus debt service plus the stock of short-term debt at the end of the last period.

$3 /$ Revenues excluding grants.

4/ Debt service is defined as the sum of interest and amortization of medium and long-term debt.

5/ Historical averages and standard deviations are generally derived over the past 10 years, subject to data availability. 
Table 2b. Kenya: Sensitivity Analysis for Key Indicators of Public Debt 2009-2029

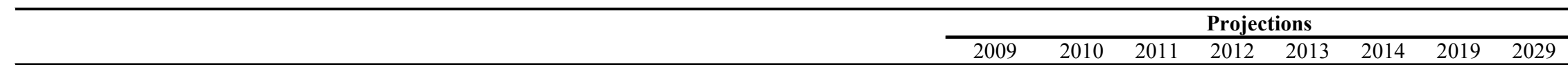

\section{PV of Debt-to-GDP Ratio}

\section{Baseline}

\section{A. Alternative scenarios}

A1. Real GDP growth and primary balance are at historical averages

A2. Primary balance is unchanged from 2009

A3. Permanently lower GDP growth $1 /$

\section{B. Bound tests}

B1. Real GDP growth is at historical average minus one standard deviations in 2010-2011

B2. Primary balance is at historical average minus one standard deviations in 2010-2011

B3. Combination of B1-B2 using one half standard deviation shocks

B4. One-time 30 percent real depreciation in 2010

B5. 10 percent of GDP increase in other debt-creating flows in 2010
A4. Alternative Scenario: Growth lower by one standard deviation 2010-2014

$\begin{array}{llllll}32 & 31 & 31 & 33 & 33 & 33\end{array}$

31

$\begin{array}{llllllll}32 & 28 & 27 & 26 & 26 & 24 & 21 & 13 \\ 32 & 31 & 33 & 35 & 36 & 37 & 42 & 47 \\ 32 & 31 & 32 & 34 & 35 & 35 & 40 & 49 \\ 32 & 32 & 33 & 35 & 37 & 37 & 41 & 49\end{array}$

PV of Debt-to-Revenue Ratio 2/

\section{Baselin}

139

136

136

142

146

145

150

136

\section{A. Alternative scenarios}

A1. Real GDP growth and primary balance are at historical averages

A2. Primary balance is unchanged from 2009

A3. Permanently lower GDP growth $1 /$

A4. Alternative Scenario: Growth lower by one standard deviation 2010-2014

$\begin{array}{rrrrrrrr}139 & 123 & 115 & 115 & 113 & 108 & 93 & 58 \\ 139 & 137 & 141 & 151 & 159 & 163 & 188 & 205 \\ 139 & 137 & 139 & 147 & 153 & 155 & 178 & 218 \\ 139 & 139 & 143 & 153 & 161 & 164 & 182 & 215\end{array}$

\section{B. Bound tests}

B1. Real GDP growth is at historical average minus one standard deviations in 2010-2011

B2. Primary balance is at historical average minus one standard deviations in 2010-2011

B3. Combination of B1-B2 using one half standard deviation shocks

B4. One-time 30 percent real depreciation in 2010

B5. 10 percent of GDP increase in other debt-creating flows in 2010

$\begin{array}{llllllll}139 & 141 & 152 & 163 & 172 & 176 & 201 & 211 \\ 139 & 129 & 125 & 132 & 136 & 135 & 143 & 133 \\ 139 & 127 & 124 & 134 & 141 & 144 & 162 & 158 \\ 139 & 148 & 147 & 152 & 155 & 152 & 152 & 136 \\ 139 & 175 & 173 & 178 & 180 & 178 & 174 & 149\end{array}$




\begin{tabular}{|c|c|c|c|c|c|c|c|c|}
\hline & \multicolumn{8}{|c|}{ Projections } \\
\hline & 2009 & 2010 & 2011 & 2012 & 2013 & 2014 & 2019 & 2029 \\
\hline \multicolumn{9}{|c|}{ Debt Service-to-Revenue Ratio 2/ } \\
\hline Baseline & 25 & 24 & 24 & 27 & 26 & 24 & 23 & 19 \\
\hline \multicolumn{9}{|l|}{ A. Alternative scenarios } \\
\hline A1. Real GDP growth and primary balance are at historical averages & 25 & 24 & 23 & 25 & 24 & 21 & 17 & 10 \\
\hline A2. Primary balance is unchanged from 2009 & 25 & 24 & 24 & 27 & 27 & 26 & 27 & 28 \\
\hline A3. Permanently lower GDP growth $1 /$ & 25 & 24 & 24 & 27 & 27 & 25 & 26 & 29 \\
\hline A4. Alternative Scenario: Growth lower by one standard deviation 2010-2014 & 25 & 25 & 25 & 28 & 29 & 28 & 28 & 30 \\
\hline \multicolumn{9}{|l|}{ B. Bound tests } \\
\hline B1. Real GDP growth is at historical average minus one standard deviations in 2010-2011 & 25 & 25 & 25 & 29 & 29 & 28 & 29 & 29 \\
\hline B2. Primary balance is at historical average minus one standard deviations in 2010-2011 & 25 & 24 & 23 & 25 & 25 & 23 & 22 & 19 \\
\hline B3. Combination of B1-B2 using one half standard deviation shocks & 25 & 25 & 24 & 26 & 25 & 24 & 24 & 22 \\
\hline B4. One-time 30 percent real depreciation in 2010 & 25 & 25 & 25 & 28 & 27 & 26 & 25 & 22 \\
\hline B5. 10 percent of GDP increase in other debt-creating flows in 2010 & 25 & 24 & 26 & 32 & 31 & 29 & 26 & 21 \\
\hline
\end{tabular}

Sources: Country authorities and staff estimates and projections.

1/ Assumes that real GDP growth is at baseline minus one standard deviation divided by the length of the projection period.

2/ Revenues are defined inclusive of grants. 


\section{IMF Executive Board Approves US\$209 Million Disbursement to Kenya Under the Exogenous Shocks Facility}

The Executive Board of the International Monetary Fund (IMF) today approved an SDR135.7 million (about US\$209 million) disbursement under the Exogenous Shocks Facility (ESF) to help Kenya recover from the negative impact of higher food and international fuel and fertilizer costs, and the slowdown in external demand associated with the global financial crisis.

The disbursement was approved under the rapid-access component of the ESF, a facility designed to provide policy support and financial assistance to low-income countries facing exogenous but temporary shocks. It is available to countries eligible for the Poverty Reduction and Growth Facility (PRGF) - the IMF's main instrument for financial assistance to low-income countries - but that do not have a PRGF-supported program in place. Financing terms are equivalent to a PRGF arrangement and are more concessional than under other IMF emergency lending facilities.

Kenya's economic recovery after political disturbances in early 2008 has been cut short by a range of factors, including the impact of rising international prices for food, fuel, and fertilizer. Further economic strain was exerted by falling global demand that has translated into weaker export growth and lower tourism receipts, remittances and private capital flows.

Food insecurity in Kenya has also increased as high fuel and fertilizer costs led to lower agricultural yields, and poor rainfall in October-November 2008 further reduced food supplies, especially of the main staple, maize. The number of people affected by food insecurity has risen by 47 percent to around 10 million, according to the government and the United Nations.

In sum, these shocks have weakened Kenya's balance of payments, threatened macroeconomic stability, and reversed progress on poverty reduction made during the 
previous five years. The IMF estimates the adverse effects of these shocks on Kenya's balance of payments at 41/2-71/4 percent of GDP - a cumulative total of US $\$ 1.4-\$ 2.2$ billion over the 2008/09 and 2009/10 fiscal years. Economic growth, which averaged 6 percent from 2004-2007, is estimated at just 2 percent in 2008 and 3 percent in 2009.

The Kenyan authorities have responded swiftly by easing monetary policy to support economic activity, reprioritizing public spending and acting to strengthen the banking system. They have also focused on improving food distribution to ensure better access to staple foods, and are working with the World Bank to introduce a well-targeted food subsidy program by the end of 2009. However, substantial increases in maize imports until at least the next food harvest in the third quarter of 2009 are expected to further erode foreign reserves and increase pressure on the Kenyan shilling.

Following the Executive Board's discussion of Kenya, Mr. John Lipsky, First Deputy Managing Director and Acting Chair, stated:

"Kenya's strong economic performance in recent years has been stalled by a series of exogenous shocks - drought; increases in international food, fuel and fertilizer prices; and the global downturn. These shocks have slowed economic growth, raised inflation, weakened the currency, and resulted in the loss of international reserves.

"The authorities have responded to the shocks swiftly. Monetary policy has been eased to support economic activity; and fiscal policy has been accommodating without endangering debt sustainability. Structural policies have focused on improving the food distribution mechanism for better access to staples, as well as on measures to strengthen the banking system. Nonetheless, there are significant risks arising from rainfall dependency, a deeper and more prolonged global downturn, and the domestic political situation. Economic policies should stand ready to respond if necessary to ensure fiscal sustainability and price stability.

"Economic policies in 2009/10 will focus on reducing inflation, rebuilding foreign exchange reserves and strengthening the basis for a return to robust sustainable growth. Fiscal policy will provide a stimulus to the economy and protect key infrastructure and social spending while ensuring that public debt levels remain sustainable over the medium term.

"Broad-based structural and governance reforms will aim to ensure strong economic growth over the medium and long term. Priority should be given to strengthening governance by developing an implementation plan for the updated Governance Action Plan, improving public financial management, and implementing financial sector reforms. Introduction of a targeted subsidy framework will address the food deficit in an efficient manner.

“In support of the authorities' policies, the IMF's Executive Board has approved Kenya's request for disbursement under the Rapid-Access Component of the Exogenous Shocks Facility. Fund support will enable Kenya to close its financing gap while improving the prospects for additional donor support," Mr. Lipsky stated. 


\section{Statement by Samuel P.O. Itam, Executive Director for Kenya May 29, 2009}

1. My Kenyan authorities thank management and staff for the constructive policy dialogue and the Board for candid policy advice. The authorities have been implementing prudent macroeconomic policies in line with their medium-term development strategy. However, the balance of payments position has been adversely impacted by a succession of exogenous shocks - the high fertilizer and fuel prices of 2008, the current global economic slowdown, and the severe drought of 2008. The authorities request disbursement under the rapid-access component of the Exogenous Shock Facility (ESF) to mitigate the adverse impact of these shocks. They believe that the shocks are sudden and temporary and are confident that their commitment to effectively implement their medium-term development strategy will aid recovery from the impact of the shocks.

2. The IMF Executive Board concluded the 2008 Article IV Consultation on September 10, 2008 during which the Directors commended the authorities for maintaining economic stability, despite the post-election turmoil, and pursuing sound macroeconomic policies while implementing economic reforms. The authorities remain committed to implement sound macroeconomic policies and the structural reform agenda, and to consolidate the government of national unity.

\section{Recent economic developments}

3. Economic performance was robust and broad-based in 2007, with real GDP growth recorded at 7 percent, anchored on a package of bold economic and structural reforms within the framework of the Economic Recovery Strategy. Hardly had the country recovered from the post-election unrest than it was hit by three external shocks, in rapid succession-high fuel and fertilizer prices, drought and the global economic contraction. The global contraction and weaker commodity prices led to a sharp slowdown in the growth of real GDP to 3.5 percent in 2007/08 and 3.0 percent in 2008/09 from 6.7 percent in 2007. Growth is projected to remain at around 3 percent for 2009/10. The slowdown in economic growth threatens to erode the significant gains made in reducing poverty in the recent past.

4. Inflationary pressures remained elevated on account of post-election shortages in the first quarter of 2008 and, thereafter, high global food and fuel prices, shortage of maize as a consequence of the drought, and depreciation of the shilling. Inflation eased slightly to 27.7 percent in December 2008 from a peak of 31.5 percent in May 2008 (noting that the CPI inflation is overstated by a factor of about 2 because of methodological problems). Annual growth in broad money supply stood at 14.9 percent in December 2008, mainly attributed to an expansion in net domestic assets of 22.9 percent on account of increased domestic credit to the private sector.

5. We wish to differ with the staff perception that "the impact of the Central Bank of Kenya's (CBK) countercyclical policy has been relatively ambiguous". The CBK monetary program has been unambiguous in helping the economy recover from the downturn associated with the political unrest earlier in 2008 and, later, the impact of the global 
contraction. These developments should also be understood in the context of other underlying dynamics in the financial sector. In particular, the outcome of the Safaricom IPO skewed liquidity in the banking system - a few banks had too much liquidity while the majority of banks experienced liquidity shortages. The CBK advised the Safaricom receiving banks to open lines of credit with other banks, while keeping a cap on interbank rates. The authorities further provided reverse repo to banks facing liquidity shortages. These measures ensured adequate liquidity and helped keep the short-end interest rates low. Moreover, the perceived economic risks associated with the global financial crisis made economic agents risk averse, who adopted "the wait and see" attitude in case the fallout from the crisis would be more severe and persistent. As a result, demand for real balances fell. Thus, in view of increased global uncertainty, the approach by the authorities was countercyclical, with a focus on dampening interest rates and providing the necessary liquidity to the banking system in order to stimulate economic activity. Given the sudden drop in economic activity, the adjustment process is still taking place, explaining why money demand is still adjusting from a low base.

6. The fiscal stance was loosened somewhat with the overall budget deficit expanding from 3.6 percent of GDP in 2007/08 to a projected 5.2 percent in 2008/09, reflecting both revenue shortfalls and expenditure adjustments in response to the economic challenges. The net domestic borrowing is expected to be much larger than anticipated mainly because of the postponed issuance of the foreign currency denominated bond on account of unfavorable external conditions, and inability to proceed with privatization in the significantly worsened economic/business environment. Although total expenditure is projected to decline, it is higher relative to revenue on account of an initial high fuel import bill which combined with subsidized importation of maize. The main fiscal challenges are to resettle people who were displaced during the elections unrest, ensure adequate supply of fertilizer and seed, and continue importing maize, even though the latter adversely affected the international reserves position.

7. The external current account deficit is estimated to widen to 6.0 percent of GDP in 2008/09 from 4.6 percent in 2007/08 due to a deterioration in the trade balance. The capital and financial account deteriorated on account of a sharp drop in foreign direct investment and intensified outflows of portfolio investment. These developments have resulted in a significant overall balance of payments deficit and depletion of foreign exchange reserves from $\$ 3,443$ million (3.6 months of imports cover) in 2007/08 to an estimated $\$ 2,796$ million in 2008/09 (equivalent to 3.1 months of imports). International reserves are projected to recover only slightly to about $\$ 3,000$ million ( 2.9 months of imports) in $2009 / 10$ compared to a statutory requirement of 4 months import cover. The financial support being requested is to alleviate this external account pressure.

8. Risks continue to be on the down side on account of high food prices, weakening global demand and commodity prices, as well as worsening drought situation with many parts of Eastern and North Eastern Kenya in dire need of humanitarian assistance - an estimated 10 million people have become food insecure and many more at risk of starvation. 


\section{Macroeconomic policy framework}

9. My authorities would like to assure the Board that they remain committed to pursuing prudent macroeconomic policies and structural reforms. In line with the objective of restoring a sustainable high growth trajectory for the economy, consistent with the Medium Term Plan and the Budget Strategy Paper, both of which are derived from the National Vision 2030. They are cognizant that economic policymaking will be challenging in the near term, given the global contraction, terms of trade shocks and weak domestic growth. These challenges notwithstanding, they remain committed to their development agenda.

\section{Fiscal policy}

10. In order to address the above challenges and ensure that the budget remains on course, the authorities have anchored fiscal policy on net domestic financing. In this regard, the measures being implemented focus on:

i. rationalizing expenditures, while safeguarding key infrastructure projects and spending on vulnerable groups;

ii. mobilizing additional revenue through strengthening tax administration, especially for excise taxes and VAT to recoup revenue loses; and

iii. seeking external financing to accommodate the additional food relief expenses and containing domestic borrowing to ensure medium-term debt sustainability.

11. Accordingly, recruitment in the public sector has been frozen for this financial year. Capital spending will also be frozen where projects have not yet commenced. With regard to supporting vulnerable groups, the authorities have withdrawn the generalized maize subsidy in preference for a new well-targeted food subsidy program in late 2009, which avoids price distortions while minimizing opportunities for abuse. The authorities are also seeking TA to improve tax administration. The authorities recognize downside risks of a larger-thanprojected revenue shortfall. Any further shortfalls in revenue will be met by corresponding cuts in spending. Moreover, the authorities have no intention of further loosening the fiscal stance beyond the target for net domestic borrowing as a way of continuing to stimulate the economy while containing domestic liquidity pressure. The above fiscal approach will avert the risk of raising interest rates and jeopardizing recovery of the private sector, as well as pursue the authorities' commitment to reduce the debt-to-GDP ratio in the medium-term.

\section{Monetary and exchange rate policies}

12. The immediate monetary policy aims at easing liquidity. The policy rate (the central bank rate) has been lowered to 8 percent and bank reserve requirements reduced to 5 percent. This will ensure adequate liquidity to support economic activity while containing domestic inflation. Monetary policy is anchored on the desire by the CBK to build up international reserves to the statutory requirements of 4 months import cover at an appropriate time, with partial sterilization of liquidity to ensure consistence with the inflation target in the mediumterm. 
13. The market determined exchange rate policy has served the country well, and the exchange rate remains broadly in line with fundamentals. In this regard, the authorities are determined to maintain this policy framework, with intervention limited to smoothing shortterm volatile fluctuations.

\section{Structural reforms}

14. The key element of Kenya's structural reform agenda is to improve competitiveness. In this regard, significant progress has been made in removing impediments to the business environment and creating an efficient and effective public sector. This includes the on-going implementation of the Governance Action Plan (GAP) — continued strengthening of the legal platform to fight corruption, operationalization of the public complaints standing committee, and development of implementation plan for GAP. Furthermore, the government has adopted risk-based auditing and rolled out the IFMIS. Finally, several reforms are being made in terms of business licenses.

15. Progress has also been recorded in the implementation of financial sector reforms in microfinance, insurance, banking and capital markets. A specific example is the Microfinance Act which is now operational. To avoid increased risk to the financial sector, more still needs to be done to strengthen and improve the competitiveness of the sector. While exposure to the global financial crisis has been limited, there has been some exposure to the regional market. The banks and authorities are closely monitoring the situation, committed and prepared to respond appropriately to avert any financial crisis. To this end, a number of measures will be introduced to make adequate provisioning and enhance the proactive approach to surveillance and enforcement through the enactment of the Banking Bill. Other measures include increasing the minimum capital requirements for banks in the next two years; enacting the Savings and Credit Cooperatives (SACCOs) and national payments bills; and selling more shares of the National Bank of Kenya to the private sector.

\section{Conclusion}

16. My authorities consider Fund support as crucial to help meet their developmental challenges in the context of the global financial crisis and economic contraction. The authorities are requesting financing under the rapid access component of the Exogenous Shock Facility in order to help increase international reserves and market confidence. They believe that the policies put in place are appropriate to address the shocks and remain ready to take additional measures should circumstances warrant. 